\title{
THINNING, MOVEMENT, AND VOLUME LOSS OF RESIDUAL CORTICAL TISSUE OCCURS AFTER STROKE IN THE ADULT RAT AS IDENTIFIED BY HISTOLOGICAL AND MAGNETIC RESONANCE IMAGING ANALYSIS
}

JENNI M. KARL

B.SC. (Hons), University of Lethbridge, 2008

\author{
A Thesis \\ Submitted to the School of Graduate Studies \\ of the University of Lethbridge \\ in Partial Fullfilment of the \\ Requirements for the Degree \\ MASTER OF SCIENCE \\ Department of Neuroscience \\ University of Lethbridge \\ LETHBRIDGE, ALBERTA, CANADA
}

(C) Jenni M. Karl, 2010 


\begin{abstract}
Plasticity of residual cortical tissue has been identified as an important mediator of functional post-stroke recovery. After neonatal stroke the thickness of residual tissue can change, the tissue can move, and tissue can fill in the stroke core. Nevertheless, the majority of preclinical stroke research utilizes adult rats. Thus, the purpose of the present thesis was to systematically document such gross morphological changes in peri-infarct tissue after stroke in the adult rat. Morphological changes were assessed in pial strip devascularization, photothrombotic occlusion, and middle cerebral artery occlusion models of stroke using histological and magnetic resonance imaging. Decreases in cortical thickness, volume, and neural density were found to extend far beyond the stroke infarct and included the sensorimotor regions of the intact hemisphere. Movement of residual tissue towards the infarct was observed and confirmed using anatomical markers placed in intact cortical tissue at the time of stroke induction. I conclude that the extensive time-dependent morphological changes that occur in residual cortical tissue must be considered when evaluating plasticity-related cortical changes associated with post-stroke recovery of function.
\end{abstract}




\section{ACKNOWLEGEMENTS}

Firstly, I would like to thank my supervisor Dr. Ian Q. Whishaw. Throughout my academic career he has provided unrivaled mentorship, insight, encouragement, patience, and support. He has afforded me both the creative freedom and knowledgeable guidance that has led to this success. I cannot express the full extent of my gratitude. I must also thank Dr. Omar A. Gharbawie who first impressed upon me the significance of behavioural research and inspired me to pursue this discipline.

This project would not have been possible without the technical expertise and assistance provided by various colleagues here at the CCBN; Dr. Mariam Alaverdashvili, who performed a number of pial strip devascularization and photothrombotic occlusion surgeries and provided insightful comments to improve the manuscript; Dr. Albert R. Cross, James Cardiff, Valerie Lapointe, and Brad Henrie who guided and assisted me in obtaining the MRI data; Jessica Cummins for her help with cortical thickness measures from a pilot study; and, Dr. Omar A. Gharbawie and Gita Gholamrezaei for providing additional samples of histological tissue. I am also grateful for the financial support provided by the Natural Science and Engineering Research Council of Canada.

A big thank-you is extended to all of the past and present members of the Whishaw lab that have helped me to critically examine my own work, provided various forms of technical assistance, and made life in the laboratory a lot of fun. Those who have not already been mentioned notably include Lori-Ann Sacrey, Diana Lim, Scott Travis, Bogdan Gorny, Crystal Erickson, Farshad Nemati, Kelly Mills, and Jill Dosso. 
I would also like to extend my gratitude to the numerous faculty and staff that make it possible for students to pursue graduate studies here at the CCBN. In particular, the animal-care staff; Karen Dow-Cazal and Charolette Holmes, as well as the administrative staff; Naomi Cramer and Donna McLaughlin.

Finally, my biggest thank-you is extended to my family; Byron and Gabriella Karl, Mark Forth, Jeff Karl, Kristen Karl, Don and Marj Karl, as well as the extended Karl, Reiser, and Forth families. Never once have they failed to support and encourage my academic and personal pursuits. For them, I am ever grateful. 
Title Page $\quad$ i

$\begin{array}{ll}\text { Signature Page } & \text { ii }\end{array}$

Abstract iii

Acknowledgements $\quad$ iv

Table of Contents vi vi vi vis

List of Figures vii

Chapter 1. Literature Review and General Introduction p.1

Chapter 2. Spatiotemporal changes in histological cortical thickness after pial strip devascularization stroke of the forelimb area of motor cortex in the adult rat

Chapter 3. Chronic changes in histological cortical thickness in common rodent stroke models of varying induction method, size, and location

Chapter 4. Spatiotemporal changes in MRI cortical thickness after pial strip devascularization stroke of the forelimb area of motor cortex in the adult rat: the contribution of tissue movement.

Chapter 5. General Discussion

References

p. 144 


\section{LIST OF FIGURES}

Figure 1. Schematic drawing of the "topographical" model of focal stroke localized to the forelimb area of rat motor cortex.

p. 3

Figure 2. Schematic drawings of the "topographical" model of focal stroke localized to the forelimb area of rat motor cortex (A) and a proposed "plastic" model of focal cortical stroke localized to the forelimb area of rat motor cortex.

p. 29

Figure 3. Histological tissue sections from a pial strip devascularization rat, showing planes and locations from which measures of cortical thickness were taken.

Figure 4. Tracing of cortical thickness in the stroke hemisphere of a pial strip devascularization rat compared to a control rat 3 days (left) and 31 days (right) after stroke A); and graphs of the change in cortical thickness in pial strip devascularization rats across one month B), and in the acute vs. chronic time periods $\mathrm{C}$ ).

Figure 5. Graphs of the change in overall cortical thickness from pial strip devascularization rats in the chronic post-stroke time period along the coronal A) and sagittal axes B).

Figure 6. Graphs of the change in cortical thickness along the coronal A) and sagittal B) axes across one month in pial strip devascularization rats.

Figure 7. A summary illustration of cortical thickness change (percent of control) in the chronic period in the stroke and intact hemispheres of pial strip devascularization rats.

Figure 8. Graphs of the change in post-stroke cortical volume across one month A), and in the acute vs. chronic time periods B).

Figure 9. A graph of post-stroke cortical tissue density (grey value index) summed over 10 cresyl violet coronal sections, encompassing the stroke infarct in pial strip devascularization rats, 31 days after stroke.

p. 61

Figure 10. Coronal sections encompassing the stroke infarct from pial strip A), small pial strip B), photothrombotic C), control D), middle cerebral artery occlusion with cortical and subcortical damage E), middle cerebral artery occlusion with only subcortical damage F), and a control G) rat, 31 days post-stroke. 
Figure 11. Cortical thickness, averaged across all coronal and sagittal planes, in the intact and stroke hemispheres from small pial strip A), pial strip B), photothrombotic C), middle cerebral artery occlusion with cortical and subcortical damage D), and middle cerebral artery occlusion with only subcortical damage E) rats, compared to controls at 31 days post-stroke.

Figure 12. Graphs of the change in cortical thickness from small pial strip devascularization rats in the chronic post-stroke time period along the coronal A) and sagittal axes B).

Figure 13. Graphs of the change in cortical thickness from photothrombotic occlusion rats in the chronic post-stroke time period along the coronal A) and sagittal axes B).

p. 86

Figure 14. Graphs of the change in cortical thickness from middle cerebral artery occlusion with cortical and subcortical damage rats in the chronic poststroke time period along the coronal A) and sagittal axes B). p.89

Figure 15. Graphs of the change in cortical thickness from middle cerebral artery occlusion with subcortical only damage rats in the chronic post-stroke time period along the coronal A) and sagittal axes B). p. 92

Figure 16. Graphs of the change in overall cortical thickness in magnetic resonance images obtained from pial strip devascularization rats across one month A) or in the acute vs. chronic time period B).

p. 107

Figure 17. Graphs of the change in cortical thickness from magnetic resonance images of pial strip devascularization rats in the chronic post-stroke time period along the coronal A) and sagittal axes B).

Figure 18. Tracings from histological tissue A) and magnetic resonance images B) from pial strip devascularization rats 31 days post-stroke illustrating the location of vertical cannula tracts in the intact and stroke hemispheres. A photomicrograph C) of a coronal section encompassing the stroke infarct from a pial strip devascularization rat, 31 days post-stroke illustrating the extent of somatosensory cortical pyramidal cell movement in the intact vs. stroke hemispheres. A graph quantifying the extent of somatosensory cortical pyramidal cell movement in the chronic post-stroke time period in pial strip devascularization rats D).

p.113

Figure 19. A schematic diagram illustrating the location of cortical thickness measures in a control, photothrombotic, and pial strip devascularization rat 31 days post-stroke (left). A graph showing the topographical change in cortical thickness along the coronal axis in these 3 different animals (right). p. 118 
Figure 20. Coronal cresyl violet sections, encompassing the stroke infarct core, from a pial strip devascularization (top) and photothrombotic (bottom) rat, 31 days post-stroke, illustrating the extent of somatosensory cortical pyramidal cell movement in the intact vs. stroke hemispheres.

p. 121

Figure 21. The traditional "topographical" model of residual cortical morphology in the adult rat (A) and the new "plastic" model of residual cortical morphology in the adult rat (B), illustrating the impact of tissue movement and thinning on identification of previously defined molecular, cellular, and electrophysiological functional cortical areas.

p. 131 


\title{
CHAPTER ONE
}

\section{LITERATURE REVIEW AND GENERAL INTRODUCTION}

\begin{abstract}
Both plastic changes and activation of pre-existing circuitry in residual cortical tissue are thought to be important mediators of behavioral compensation and recovery after stroke. Adult rat stroke models are often used to model the human condition. Currently, the extent of morphological change that occurs after stroke in the adult rat has not been systematically documented, and thus it is not known how post-stroke morphological changes may be impacting molecular, cellular, and electrophysiological investigations of residual cortical tissue function. In the following review I first describe the molecular, cellular, and electrophysiological features traditionally used to define the gross organization of the adult rat brain after stroke, ie. the stroke infarct, penumbra, and distal cortical areas. Secondly, I consider the morphological changes known to occur in residual neonatal rat brain, as these may provide insight into the type of changes that may occur in the adult rat. Next, I propose the investigation of a new "plastic" model of rat cortex after stroke for the examination of residual tissue function. This "plastic" model takes into consideration the possibility of gross morphological change after stroke in the adult rat brain. Finally, I discuss the main objectives for the present thesis.
\end{abstract}




\section{The "Topographical" Model of Rat Cortical Morphology after Stroke}

Focal cortical stroke in the adult rat brain is commonly used to model a number of aspects of human stroke. The current model of rat cortex after stroke is based on the topographical development of a clearly identifiable stroke infarct, penumbra, and distal residual brain regions. The stroke infarct is a region where neural tissue suffers irreparable ischemic damage and ultimately dies. The penumbra includes tissue that is directly exposed to the ischemic insult but damage may be reversible. Distal brain regions include residual neural tissue not directly exposed to the ischemic insult but that may function abnormally after stroke. A schematic depiction of this "topographical" model is shown in Figure 1.

The topographical model has been used to investigate two important aims of current brain research. Firstly, the function of a localized brain region is traditionally determined by observing the specific functional and/or behavioural deficits that occur after damage to that localized (infarcted) region. Secondly, molecular, cellular, and electrophysiological changes in residual tissue are studied to determine their contribution to post-stroke functional and behavioural recovery. Both of these methods are based on the topographical model and assume that the gross morphology of residual cortex is unchanged and does not influence experimental findings.

In the following sections I will describe the molecular, cellular, and electrophysiological features traditionally used to define the location of each post-stroke topographical region. 


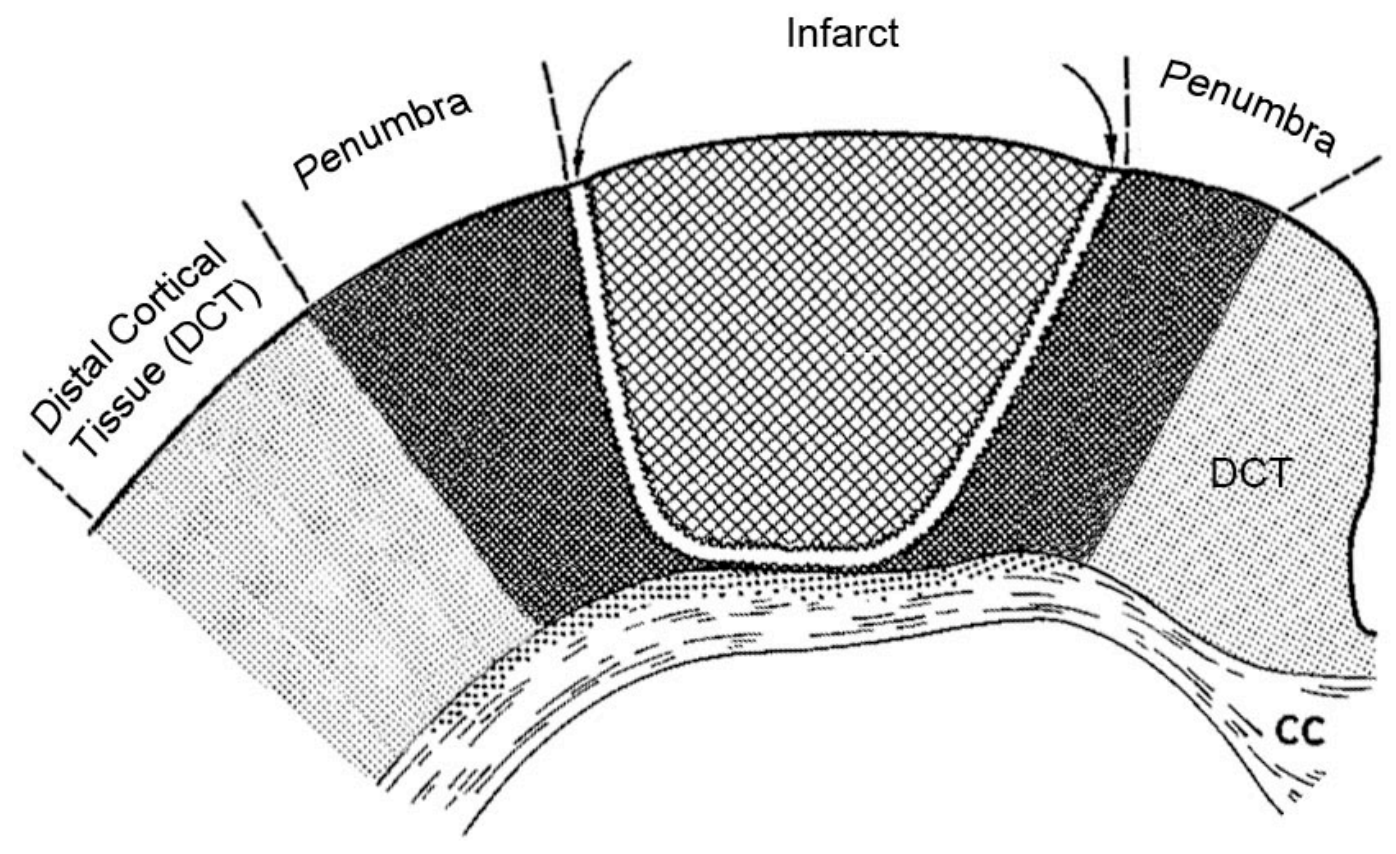


Figure 1. The traditional topographical model of rat cortical morphology after stroke. Three distinct topographical regions are thought to develop; the stroke infarct is centrally located, surrounded by the penumbra, and then distal cortical tissue. CC - corpus callosum; DCT - distal cortical tissue. Adapted from Bidmon et al., 1997. 


\section{Molecular, Cellular, and Electrophyisological Features of the Stroke Infarct, Penumbra, and Distal Cortical Regions as Defined by the "Topographical" Model}

\section{The Stroke Infarct}

The stroke infarct is defined as the brain region that sustains irreversible ischemic damage. Ischemia is produced by a blockage or breakage in the blood vessel that irrigates the affected cerebral region resulting in an inadequate supply of blood, oxygen, and nutrients necessary to maintain normal tissue metabolism. In the following sections I will describe the subsequent cascades of molecular, cellular, and electrophysiological events that result in fast and irreversible tissue death, clearance, and cavity formation within the stroke infarct.

\section{Molecular Changes}

Cell death within the stroke infarct results from abrupt and severe disruptions in metabolism. This local energy deficit, caused by a drastic drop in ATP (Adenosine Triphosphate), leads to the activation of voltage-gated calcium channels, which produces an accumulation of excitotoxic amino acids (ie. glutamate) in the extracellular compartment. This excess of glutamate leads to the activation of NMDA (N-methyl-Daspartic acid) and AMPA ( $\alpha$-amino-3-hydroxy-5-methyl-4-isoxazolepropionic acid) receptors on surrounding cells causing an even further increase in intracellular calcium, 
sodium, and chlorine ion concentrations. This excessive disturbance in ion homeostasis results in the passive influx of water into the cell, causing the cell membrane to stretch, tissue edema, and osmotic lysis of the cell (Mergenthaler et al, 2004). This form of cell death is traditionally known as necrosis. Although, recent evidence suggests that necrotic cell death may be more programmed than previously thought.

Self-initiated necrosis has recently been described in ischemic tissue (Zong and Thompson, 2010). The metabolic disturbances described above signal the onset of selfinitiated necrosis. Unlike the traditional form of necrosis, self-initiated necrosis is dependent on the activity of specific genes within the cell, which leads to suicidal but non-apoptotic cell death in the stroke infarct. For instance the gene that encodes, cyclophilin $\mathrm{D}$, a component of the mitochondrial permeability transition pore actively participates in degradation of the necrotic cell. Self-initiated necrosis appears to be an adaptive response to ischemic injury that contributes to inflammatory and wound repair processes that help to maintain homeostasis and tissue integrity.

it occurs very quickly and is the major form of cell death within the stroke infarct.

The production of free radicals is another mechanism by which tissue damage occurs in the stroke infarct (Dirnagl et al., 1999; Sims and Anderson, 2002). A number of free radicals are produced as a result of excitotoxicity and can damage DNA, phospholipid membranes, organelle compartments, and almost any other aspect of the cell. Free radical damage can shut down mitochondrial functioning, further diminishing cellular energy supplies (ATP), and also cause the release of pro-apoptotic molecules. Pro-apoptotic molecules facilitate apopotosis (cellular suicide) in surrounding cells. 


\section{Cellular Changes}

Severe focal ischemia $(<10 \%$ of normal blood pressure) occurs in the stroke infarct and leads to perturbations in cellular morphology prior to cell death. Different labeling methods including carbocyanine DiI, yellow/green fluorescent protein, and Golgi-Cox staining have been used to describe the beaded morphology of ischemic neuronal dendrites (Hasbani et al., 2001; Park et al., 1996). Zhang and colleagues (2005) show that dendritic tufts and spines located within the infarct undergo severe morphological change and swelling within 10 minutes of ischemia onset. Interestingly, rapid reperfusion of the infarct core (within 20-60 minutes) leads to significant morphological recovery. The authors suggest that the application of pharmacological treatments, such as tissue plasminogen activators, within this time period may be able to facilitate cellular survival, not only in the penumbra, but also within the stroke core. Twenty-four hours after stroke onset, however, spines and dendrites that had initially recovered began to degenerate again. The authors suggest that this subsequent degeneration may have been due to initiation of a secondary toxic process.

Astrocyte degeneration also occurs within the stroke infarct (Landis, 1994). Osmotic-induced swelling of astrocytes has been noted as an early indicator of ischemia and can exacerbate cerebral damage by increasing intracranial pressure and further reducing cerebral blood flow.

\section{Electrophysiological Changes}


Limited information is available concerning the dynamic electrophysiological properties of neurons undergoing direct ischemia in the stroke infarct. One study (Chiganos et al., 2006), however, used implanted microwire electrodes to track the dynamic electrophysiological responses of rat auditory neurons directly exposed to photothrombotic ischemia. They used the extinction of electrical responses in ischemic tissue as an indicator of cell death. Extinction of electrical responses in ischemic tissue took a variable time course with some neurons quiescent by 300 seconds post-ischemia, and others lasting up to 600 seconds.

\title{
The Stroke Penumbra
}

The stroke penumbra includes tissue immediately surrounding the stroke infarct. Penumbra tissue suffers ischemia to a lesser degree than tissue within the stroke infarct. Tissue damage in this area develops much slower and some penumbral tissue may eventually be incorporated into the stroke infarct while other tissue may be rescued by improved metabolism and blood flow from residual collaterals within this tissue. The penumbra zone is heterogeneous in that the molecular, cellular, and electrophysiological responses within this tissue vary from region to region. I discuss these responses in the following sections.

\author{
Molecular Changes
}


While the major form of cell death within the stroke infarct is necrosis, apoptotic cell death and inflammatory processes are the major players in the penumbra. Anoxic and excitotoxic depolarizations within the stroke infarct can lead to excessive extracellular glutamate and potassium concentrations, which can then diffuse into neighboring penumbral tissue. This diffusion propagates a wave of "peri-infarct" depolarization away from the stroke core, termed spreading depression (Back et al., 1996; Nedergaard and Hansen, 1993). These depolarizing waves can occur at a frequency of 1 to 4 per hour (Wolf et al., 1997). Because repolarization is an energy-dependent process, this may further stress the already ischemic cells, leading to growth of the stroke core into the penumbral tissue.

Apoptosis in the penumbra starts hours after ischemia onset and lasts for days. In addition to waves of spreading depression, DNA damage, excess glutamate, free radical species, and elevated intracellular calcium levels are other activators of apoptosis in the penumbra. Ultimately these activators lead to the release of cytochrome $\mathrm{c}$, damage of the mitochondrial membrane, and the activation of caspases. Caspases can activate cytokines (Ferrer and Planas, 2003), exacerbating inflammatory responses in the penumbra, and also metabolize important DNA-repair enzymes (Le et al., 2002). This leads to the destruction of critical genetic information. Meanwhile other caspases cleave structural proteins, important components of the nucleus and the cell (Endres et al. 1999), leading to the programmed demise of the cell.

Another important mediator of cellular physiology and viability in the penumbra is the extent of post-stroke brain swelling and edema. The expression of aquaporin-4 and -9 has been found to significantly increase in infarct and penumbral tissue up to 24 hours 
after stroke (Ribeiro et al., 2006; Yang et al., 2009). Aquaporins are water channel proteins that facilitate diffusion of water through astrocyte and neuronal cell membranes. This water diffusion causes the cells to swell and cerebral edema, which increases brain volume and intracranial pressure, further impairing vascular perfusion (Klatzo, 1985). These processes facilitate expansion of the infarct to include additional penumbral tissue.

It is important to note that not all cells in the penumbra are destined to become part of the ischemic core, but can be rescued if reperfusion occurs in a timely manner. During ischemia, general protein synthesis (Thilmann et al., 1986), and gene expression (Nowak, 1985; Jacewitz et al., 1986) are suppressed in the penumbra, such that only select groups of proteins such as heat shock proteins (Lindquist and Craig, 1988), immediate early genes (Kinouchi et al., 1994), neurotropins (Lindvall et al., 1992), and some apoptosis genes (Chen et al., 1995) are expressed. Some of these gene products may lead to cell death, but others can help injured neurons survive the recovery process. In penumbral tissue that can be recovered, this general suppression is transient. The potential to reverse tissue damage in the penumbra is limited to only a few hours.

\section{Cellular Changes}

Although many of the molecular hallmarks of apoptosis are identified in the penumbra in experimental stroke, the histological markers such as membrane blebbing, apoptotic bodies, condensed chromatin, etc., are not always evident. Thus, the word "apoptosis" in the penumbra is a controversial term with some stroke researchers. Nevertheless, convincing work has been done to show that characteristically apoptotic 
DNA fragmentation, and cellular condensation occurs in the penumbra up to 4 weeks after middle cerebral artery (MCA) occlusion in the rat (Enright et al., 2007; Li et al., 1995).

It has recently been shown that changes in neuronal morphology are not uniform within the penumbral zone. Enright and colleagues (2007) used in vivo 2-photon imaging and histological preparations to visualize changes in dendritic, axonal, and soma morphology in peri-infarct cortex up to 10 hours after photothrombotic or MCA occlusion stroke. They found that apical dendrites in the penumbra zone are more susceptible than deeper basal dendrites to beading and degeneration if early reperfusion does not occur. This finding supports earlier studies by Gonzalez and Kolb (2003), which show that apical dendritic tufts are more susceptible to morphological modification after aspiration stroke. Enright and colleagues also found that swelling and beading of penumbral axons extend well beyond the ischemic interface and the severity and spatial extent of this damage progressed over time. Condensation of cell bodies was also evident in both the stroke infarct and penumbra. Markers of neural necrosis and apoptosis indicated that the spatial and temporal activation of these two cell death mechanisms were similar, suggesting that both mechanisms participate in early degeneration of the infarct and penumbra zone.

Besides neuronal changes, residual microglia are also strongly activated in the penumbra. These microglia can produce a number of proinflammatory cytokines and free radicals (Gregerson et al., 2000), which can further damage penumbral tissue. Microglia are also phagocytotic and can clear dead neural tissue from the penumbra, leaving behind a glial scar (Stoll et al., 1998). Not only are residual microglia activated, but monoyctes 
from the blood can migrate into the penumbral area and by 14 days post-stroke one third of these monocytes differentiate into microglia (Priller et al., 2001). It is important to note that microglia may also have a neuroprotective role as they can release growth factors (Lee et al., 2004; Narumiya et al., 1998) long after the formation of the stroke infarct. Thus, it is likely that microglia may play both a destructive and a protective role at different post-stroke time periods, with the protective role occurring days and even weeks after the initial insult.

\section{Electrophysiological Changes}

Diffusion of potassium ions and glutamate from the stroke infarct triggers spreading depression (Branston et al., 1977). Although initiated in the penumbra, these electrochemical waves can spread to distal cortical tissues as well. Spreading depression may exacerbate pathological mechanisms by propagating ionic imbalances, and releasing glutamate at increasingly distal cortical locations. Spreading cellular depolarizations can last 1 to 5 minutes and the incidence of spreading depression has previously been correlated with infarct size (Dijkhuizen et al., 1999). Hartings and colleagues (2003) have described two waves of spreading depression after MCA occlusion stroke in the rat. The first wave occurs during the 2 hour post-stroke period, after reperfusion a 6 to 8 hour quiescent period occurs, followed by a second wave of spreading depression that lasts for another 12 hours. The authors suggest that the second wave of spreading depression may recruit penumbral tissue into the infarct core even after sustained reperfusion. Other 
research suggests however, that spreading depression may induce ischemic tolerance in remote brain areas (Kobayashi et al., 1995).

\section{Distal Cortical Tissue}

Distal brain regions are not directly exposed to ischemic conditions, and are located outside of the stroke infarct and penumbra. After stroke however, distal residual brain tissue can also undergo numerous changes that can influence eventual post-stroke behavioural and functional outcomes. The majority of changes in distal cortical tissue are thought to support functional and behavioural compensation/recovery. It is also possible however, that these changes could produce functional deficits, unique from deficits caused by cell loss localized within the stroke infarct. In the following sections I will describe the molecular, cellular, and electrophysiological changes that occur in distal residual tissue after stroke.

\section{Molecular Changes}

Changes in protein transcription and translation are not isolated to the penumbra but also extend to remote, non-ischemic cortical regions. Acute upregulation of immediate early genes (IEGs) in distal cortical tissue mirrors the temporal and spatial activation of IEGs in the penumbra, suggesting that early changes in IEGs are mediated by spreading cortical depression, rather than localized reductions in cerebral blood flow (Johansson et al., 2000). These IEGs may signal the onset of apoptosis in distal cortical 
tissue or the initiation of plastic processes for promoting tissue resilience and functional recovery.

Alterations in neurotrophic factor expression are observed in cortical tissue large distances from the stroke infarct. Both basic fibroblast growth factor (bFGF) and brain derived neurotrophic factor (BDNF) are known to be upregulated in distal astrocytes and neurons between 1 and 3 days post-stroke (Lippoldt et al., 1993; Madinier et al., 2009; Speliotes et al., 1996; Wei et al., 2000). Increased neurotrophic factor expression is thought to promote dendritic and axonal sprouting in residual cortical tissue in order to support compensatory recovery. Growth-promoting genes are also upregulated in periinfarct cortex after stroke (Carmichael et al., 2005). The highly regulated temporal expression of growth-promoting and growth-inhibiting genes reduces peri-neuronal nets and increases peri-infarct axonal sprouting. Thus, it is plausible that increased neurotrophic factor expression in residual cortex may support the development and maturity of new neuronal connections after stroke.

\section{Cellular Changes}

Vast changes in cellular morphology occur in distal cortical tissue after stroke. These changes include synaptogenesis, axonal and dendritic sprouting, neurogenesis, and astrocytic changes. These processes are highly regulated by both the pathological events of stroke induction as well as post-stroke behavioural experience. It has been suggested that external manipulations used to regulate these processes may improve post-stroke functional recovery/compensation. 
Synaptogenesis, axonal, and dendritic sprouting are thought to be dependent on the interaction of stroke infarct formation and behavioural experience. For instance, Jones and Schallert (1994) describe increases in dendritic arbour in layer V pyramidal neurons of the intact hemisphere after focal cortical stroke of the contralateral motor cortex. This increase in arbour is temporary and dependent on use of the forelimb ipsilateral (nonaffected) to the stroke infarct. As use of the affected forelimb is restored, pruning of the dendritic arbour in the intact hemisphere occurs. Subsequent studies confirmed that initial dendritic and axonal pruning in the intact motor cortex is followed by the addition of functional synaptic connections. Additionally, experience-dependent synaptogenesis is known to extend to other behaviourally relevant areas of cortex including the hindlimb representation of sensorimotor cortex in both the intact and stroke hemispheres (Allred and Jones, 2004; Chu and Jones, 2000; Hsu and Jones, 2004; Jones et al., 1996; Jones, 1999; Luke et al., 2004). Novel horizontal axonal cortical connections have also been observed in post-stroke peri-infarct tissue (Carmichael et al., 2001). Dendritic and axonal sprouting in residual cortical tissue can be modulated by tactile stimulation (Gibb et al., 2010), motor skill training (Biernaske and Corbett, 2001; Kleim et al., 2004), environmental enrichment (Johansson and Belichenko, 2002; Kolb and Gibb, 1991a), and pharmacological manipulations (Ramic et al., 2006).

Another possible cellular change is the birth, migration, and maturation of new neurons (Abrahams, 2009; Keiner et al., 2009; Kuge et al., 2009; Wurm, et al., 2007; Xue et al., 2009; Zhao et al., 2009). By co-labeling cells with BrdU and NeuN, it has been suggested that neural progenitor cells might migrate from the dentate gyrus and subventricular zone into the overlying ischemic cortex. Mature BrdU neurons can be 
found in the cortex up to 8 weeks after stroke. The application of neurotrophic factors, behavioural experience, and spreading depression may enhance post-stroke neurogenesis. Processes, other than the birth and migration of new neurons, could also contribute to the identification of BrdU labeled neurons in the mature rat cortex after stroke. Firstly, damaged cortical cells may undergo a number of DNA repair processes after stroke, which could incorporate BrdU into already mature, cortical cells (Landgren and Curtis, 2010). Secondly, some evidence suggests that mature astrocytes, already localized in the cortex, may actually change their cellular fate in response to ischemia, such that they take on a neuronal phenotype (Landgren and Curtis, 2010). Such a change could also incorporate BrdU into an already mature cortical cell. Finally, immunofluorescence is the method used to visualize co-labeled BrdU and NeuN cells in the rat cortex after stroke. Unfortunately, damage to neural tissue, as occurs in stroke, can lead to autofluorescence; non-specific, broad spectrum background fluorescence, which can lead to the overestimation of the number of new co-labeled cells in the cortex (Spanswick et al., 2009). Thus, it is not yet clear to what extend newborn neurons may migrate from the subventricular or subgranular zone to the overlying cortex of the adult rat after stroke.

Glial cell populations also change in response to focal cortical stroke in the adult rat. By examining GFAP expression, it has been found that while increases in microglial expression occur near the infarct and in penumbral tissue, large increases in astroglia occur in both proximal and distal residual tissue (Nowicka et al., 2008). The microglia response is observed only in the acute post-stroke period and is thought to be involved in glial scar formation and removal of degenerating debris in and near the infarct. The widespread astroglial response however, lasts up to 60 days after stroke and is thought to 
mediate plastic processes in residual tissue by increasing the availability of various neurotrophic factors.

\section{Electrophysiological Changes}

Functional post-stroke recovery is thought to be mediated by plastic properties of surrounding residual tissue. Indeed, intracortical microstimulation and fMRI studies have found that electrophysiological maps can reorganize after cortical stroke in order to compensate for the functional loss in the stroke infarct. Better behavioural outcome is also correlated with the extent of cortical map reorganization (Castro-Alamancos and Borrel, 1995; Friel et al., 2000; Frost et al., 2003; Gharbawie et al., 2005; Kleim et al., 2003; Nudo and Milliken, 1996). This cortical reorganization may be mediated by two mechanisms. Firstly, the activation of previously latent neural networks in residual tissue may take on the function of the lost tissue (Sigler et al., 2009); alternatively, enhanced axonal sprouting, synaptogenesis, and dendritic spine turnover (Biernaskie and Corbett, 2001; Brown et al., 2007, 2008; Gonzalez and Kolb, 2003; Stroemer et al., 1995; Winship and Murphy, 2009) may support reorganization of residual tissue such that it can support the function of the lost tissue. Again, the application of plasticity promoting factors such as environmental enrichment (Williams et al., 2006), behavioural therapy (Adkins et al., 2006; Kleim et al., 2004), pharmacological treatments (MacDonald et al., 2007), and neurotrophic factor application (Monfils et al., 2005) have been shown to facilitate reorganization of cortical maps. 
Cortical spreading depression, while it originates near the infarct in the penumbra, also extends to, and has similar impacts on, distal cortical tissue. A related electrophysiological property of residual cortical tissue is "diaschisis" (BuchkremerRatzman et al., 1996; Reinecke et al., 1999; von Monakow, 1914). Diaschisis is used to describe the temporary loss of neural excitation in undamaged and remote, but functionally related cortical areas. Diaschisis effects can spread throughout the stroke hemisphere and even transcallosally. These effects are thought to mediate the widespread deficits that are sometimes seen after focal cortical insults. Post-stroke functional recovery may be partially due to the alleviation of diaschisis and re-activation of remote brain areas.

\section{Gross Morphological Changes}

A comprehensive understanding of post-stroke morphological change in the adult rat is not yet available, but is essential for ensuring the validity of future peri-infarct experimental investigations. Any change in the size, shape, or location or post-stroke residual tissue could lead to the erroneous comparison of non-homologous tissue in stroke vs. control brains. Some morphological changes have been noted in the literature, including thinning of residual cortical tissue immediately proximal to the stroke infarct following bilateral motor cortex stroke (Kolb et al., 2000) and distortion of peri-infarct tissue into the infarct following unilateral motor cortex stroke (Whishaw, 2000). The extent, nature, and development of these changes have not been systematically described and warrant further investigation. Some insight, as to what morphological changes might 
be expected after stroke in the adult rat, may be gained by reviewing the morphological changes known to occur after neonatal stroke. These changes are discussed below.

\section{Cortical Thinning after Neonatal Stroke}

During the 1930's the Kennard Principle dominated popular scientific belief and states that the younger the age at which brain injury occurs, the better the functional outcome (Kennard, 1936). This led to vigorous investigation of behavioral recovery after neonatal stroke, and although the validity of this principle has since been questioned, it is also the reason why so much of the literature on morphological change in the residual brain is based on neonatal animal stroke models.

A series of studies by Kolb and colleagues (Kolb, 1987; Kolb et al., 1983a,b, 1994, 1995, 1998; Kolb and Cioe, 2000, 2003; Kolb and Gibb, 1990, 1991a, 1993; Kolb and Tomie, 1988; Kolb and Whishaw, 1985), systematically describe the effects of neonatal cortical stroke, via aspiration removal, on adult behaviour and brain morphology. In these studies, cortical thickness is consistently used as a measure of gross morphological change. As discussed below, the results of these studies indicate that age, stroke location, and stroke size all contribute to changes in neonatal post-stroke morphology.

\section{The Effect of Age on Post-Stroke Morphological Change in Neonatal Rats}


In general, the earlier the age of stroke induction, the greater the reduction in residual cortical thickness (Kolb, 1987; Kolb et al., 1998). When compared in adulthood, rats that receive focal cortical stroke on postnatal day 1 (P1) show greater thinning of residual cortical tissue compared to P10 animals, which in turn have more thinning than animals that receive stroke in adulthood. In addition, neonatally operated rats show abnormal organization and reduced volume of the thalamus compared to adult operated rats as a function of age of stroke induction. In all of these early studies animals that received stroke in adulthood were not shown to differ from control rats in terms of residual cortical thickness.

These results are surprising in that they do not parallel the behavioural findings. Behavioural sparing occurs after P10, but not P1 or adult stroke. Thus, it was expected that gross morphological changes in the brain would mirror these results. The fact that P10 animals show behavioural sparing, despite reduced cortical thickness, suggests that cortical thickness alone is not a substrate for the sparing of behaviour after P10 cortical stroke.

In regards to the general observation that stroke induced earlier in life results in increased cortical thinning, there is one exception; rats that receive medial frontal cortex stroke even earlier, before birth, on embryonic day 18 (E18), have normal cortical thickness compared to adult operated and adult control rats. Furthermore, these animals are indistinguishable from control animals on behavioural tasks in adulthood. This sparing of behaviour and cortical thickness is seen in prenatally operated rats, despite the presence of other types of abnormal cortical morphogenesis including reduced brain weight, abnormal growth of white and grey matter patches in the cortical lamina, as well 
as the development of neuronal bridges between the two cerebral hemispheres. Thus, stroke at an earlier age is correlated with greater cortical thinning, with the exception of prenatal (E18) stroke.

\section{The Effect of Infarct Location on Post-Stroke Morphological Change in Neonates}

Stroke location is also an important factor in determining the extent of post-stroke cortical thinning in neonates. Early studies indicate that neonatal bilateral frontal cortex stroke, bilateral posterior parietal cortex stroke, and unilateral hemidecortication have different and opposing effects on the morphology of residual cortical tissue in adulthood (Kolb et al., 1983a,b; Kolb, 1987; Kolb and Tomie, 1988).

The earliest studies show that frontal cortex stroke produces significant thinning of residual posterior parietal cortex. This was initially not seen after posterior parietal cortex stroke, however, which did not alter the thickness of residual frontal cortex. Later studies did find thinning of frontal cortex after posterior parietal lesions. It is not clear what factors may contribute to the different results found in these studies.

Neonatal hemidecortication results in the thickening of cortex in the intact hemisphere while adult hemidecortication results in thinning of cortex in the intact hemisphere. These are the first indications that residual cortical tissue can thicken after neonatal stroke, or change at all after adult stroke. Interestingly, neonatal frontal lesions and neonatal hemidecortication have the same effect on behavioural sparing despite opposite effects on residual cortical morphology. Thus, the location of the stroke can 
differentially affect residual cortical morphology, while producing similar effects on behavioural sparing in adulthood.

Despite comparable reductions in cortical thickness in adulthood, the rate at which cortical thickness declines after neonatal stroke is also dependent on the location of the infarct. There is an immediate decrease in residual cortical thickness 24 hours after $\mathrm{P} 1$, but not P5, frontal cortex stroke. Cortical thickness in P1 rats gradually increases until adolescence $(\sim \mathrm{P} 60)$ at which time a large decrease again occurs. Neonatal posterior parietal stroke does not cause an immediate drop in cortical thickness, but rather a gradual decline throughout adolescence.

Together these findings indicate that the location of a neonatal stroke infarct influences the degree, location, and rate of cortical thinning in other cortical areas.

\section{The Effect of Infarct Size on Post-Stroke Morphological Change in Neonates}

The infarcts produced in the aforementioned studies were quite large, removing almost half of the cortical mantle. More recent investigations have suggested that smaller, more focal, cortical strokes produced between P1 and P10 have the general effect of reducing residual cortical thickness; but the location of the smaller stroke infarct does influence the location of residual thinning (Kolb et al., 1995; Kolb and Whishaw, 1985; Kolb and Cioe, 2000, 2003).

Focal neonatal lesions of the motor cortex or the medial frontal cortex cause widespread thinning of residual cortex. Focal stroke localized to the posterior parietal cortex, occipital cortex, or the temporal cortex result in thinning only in cortical tissue 
located in the same plane, posterior planes, or immediately proximal planes. "Large lesions" of the medial frontal cortex on P2 produce widespread thinning throughout the remaining cortex, whereas "small lesions" induce thinning in only a single plane, most distal to the infarct (Kolb and Cioe, 2000).

Taken together the results of these studies confirm that cortical thinning, as well as other gross morphological changes, do occur in intact peri-infarct tissue after stroke in the neonatal rat brain. The severity and spatial extent of this thinning is dependent on neonatal age, infarct location, and infarct size.

\section{Possible Mechanisms of Residual Cortical Thinning after Neonatal Stroke}

There are a number of mechanisms that could be contributing to cortical thinning after neonatal stroke, but the extent to which each may contribute to the overall effect is far from clear. Neural development in the rat is incomplete at birth, thus it is almost certain that stroke-induced disruption of normal cerebral development largely contributes to post-stroke cortical thinning. In the following sections I discuss developmental abnormalities that could contribute to cortical thinning. These include disrupted cortical connectivity, synaptogenesis and dendritic branching, neurogenesis, neuronal migration and maturation, as well as residual tissue stretching and cryoanesthesia. Secondary neuronal degeneration, which is known to occur after stroke in the adult brain, could also contribute to cortical thinning after neonatal stoke, and will be discussed later.

Abnormal Cortical Projections and Connectivity 
Initially cortical thinning was only observed after neonatal frontal cortex stroke, but not posterior cortex stroke. It was thought that the frontal cortex might develop first and be a necessary precursor for proper development of posterior cortex (Kolb et al., 1983a). This was a logical argument, as it is known that subcortical noradrenaline projections course up through prefrontal cortex before projecting back towards posterior cortex (Morrison et al., 1979; Schlumpf et al., 1980). Noradrenaline has previously been shown to promote plasticity during development and depletion of noradrenaline attenuates behavioural sparing after neonatal frontal cortex stroke (Kasamatsu and Pettigrew, 1976; 1979; Schlumpf et al., 1980; Sutherland et al., 1982; Whishaw et al., 1986b). Thus, it was thought that removal of frontal cortex, and the noradrenaline projections, early in development might lead to noradrenaline depletion and cortical thinning in posterior cortex. Subsequent anatomical studies found, however, that thinning of residual cortex occurs after both frontal and posterior cortex stroke (Kolb, 1987). Furthermore, rats that received frontal lesions on P1 had additional thalamo-cortical, amygdalo-cortical, and nigro-cortical connections to residual cortex, whereas P10 and adult operated rats, with greater cortical thickness, did not. These exuberant connections were present in the normal neonatal brain. Their presence in the P1-operated brain in adulthood suggests that neonatal injury prevented normal retraction of these projections (Kolb and Gibb, 1990, 1991a, 1993; Kolb et al., 1994). These results suggest that the loss of nonspecific or subcortical projections is not a likely cause of residual cortical thinning after neonatal stroke. 


\section{Changes in Dendritic Branching and Spines}

A decrease in the density of residual neuronal processes has also been proposed to contribute to cortical thinning after neonatal stroke. Dendritic density increases dramatically after P10 stroke, slightly after adult stroke, and actually decreases after P1 stroke (Kolb and Gibb, 1991b, 1993). Thus, after P10 stroke, cortical thinning is observed in conjunction with increased dendritic density. Thus, cortical thinning is likely not due to a decrease in dendritic arborization.

\section{Disrupted Neurogenesis, Neuronal Migration and Maturation}

Disruption of neurogenesis and neuronal migration might contribute to cortical thinning after neonatal stroke. Neuronal migration is known to continue well into the first week of life in the rat (Bayer and Altman, 1991; Hicks and D'Amato, 1968) and it has previously been reported that migration is disrupted after small cortical lesions in the neonatal rat (Hicks and D'Amato, 1968). Kolb and colleagues (Kolb and Holmes, 1983; Kolb and Whishaw, 1985), document in many of their studies that the size of the stroke infarct appears much smaller in neonatal operates compared to adult operates, despite their best efforts to standardize stroke size across all groups. One possible explanation is that the stroke core was filled with newborn neurons after the stroke, which indeed occurs after P10, but not P1 or adult stroke (Kolb et al., 1998). The new neurons filling the stroke infarct may have been born to fill the infarct or they may have originally been destined for other cortical areas and re-routed to the injured area. If the latter were shown 
to be true, this could contribute to thinning of residual cortical areas, at least in the P10, but not P1 animals. The spontaneity of this effect may be specific to the medial frontal cortex however, as spontaneous regeneration was not found after stroke in the adjacent motor cortex (Kolb et al., 1998). Furthermore, it is not clear whether artificial administration of basic fibroblast growth factor is a necessary requisite for this phenomenon, as spontaneous regeneration was not consistently found after P10 medial frontal stroke (Kolb et al., 1998; Monfils et al., 2005).

In relation to the idea of disrupted neuronal migration after neonatal stroke, Kolb and Gibb (1993), report that incidental damage to the subventricular zone often occurs when operating on P1 rats, and to a much lesser degree in P10 rats during frontal cortex stroke surgery. Because the subventricular zone is a site of active neurogenesis it is possible that normal neurogenesis and migration processes are disrupted after neonatal lesions and may be exacerbated in the P1 animals. This difference could contribute to the increased cortical thinning observed after P1 neonatal stroke.

\section{Stretching of Residual Cortex}

Another possible explanation for the "filling in" of neonatal stroke infarcts is that cerebral edema and a loss of structural support may have caused peri-infarct tissue to shift and "fall" into the infarct. This proximal movement of tissue could cause stretching and subsequent thinning of distal residual cortex. This is especially likely after P1 stroke, where growth of new neural tissue in the stroke infarct does not occur, yet infarct size in adulthood is much smaller than expected and cortical thinning is the most profound. 
Initial movement of tissue into the infarct could be exasperated by the method of stroke induction used in the Kolb studies. Aspiration lesions remove the entire stroke core at once, this could lead to an immediate loss of structural support for surrounding tissue. Indeed, 24 hours after stroke, P1 and P10 brains sustain an immediate drop in cortical thickness (Kolb, 1987). Because of the short time frame, this effect cannot be due to disrupted neurogenesis and/or abnormal developmental factors. Additional thinning of residual cortex does occur over time; thus, both disrupted developmental processes and time-dependent movement of peri-infarct tissue likely contribute to this subsequent thinning.

\section{Cryoanesthesia}

A common method of neonatal anesthetization, and the method used by Kolb and colleagues, is cryoanesthesia, or cooling of the body core. This method is often chosen because it is easy to administer and pups recover quickly. Cryoanesthesia also reduces blood pressure and minimizes bleeding during surgery. Unfortunately, cryoanesthesia alters behaviour and brain morphology when administered on P1 but not P10 (Nunez et al., 1998, 2000). Both cortical thinning and decreased dendritic arborization occur after P1 cryoanesthesia (Kolb and Cioe, 2001). These anesthesia-induced morphological changes may contribute to the impaired functional outcome observed after P1 stroke, but don't seem to affect P10 outcome. Nevertheless, cryoanesthesia is likely one factor that does contribute to the decrease in cortical thickness observed after P1 stroke. 


\title{
Considerations for the Investigation of a New "Plastic" Model of Adult Rat Cortex
}

\begin{abstract}
After Stroke
Some morphological changes have been noted in the literature after stroke in the adult rat. These include thinning of cortical tissue immediately proximal to the stroke infarct following bilateral motor cortex stroke (Kolb et al., 2000) and distortion of periinfarct tissue into the infarct following unilateral motor cortex stroke (Whishaw, 2000). These findings suggest that post-stroke morphological changes may be more prevalent in the adult rat than may have previously been thought.

It is conceivable that changes in adult cortical morphology may parallel the morphological changes observed after neonatal stroke, to the exclusion of changes that result directly from disrupted developmental processes. Disrupted neurogenesis, neuronal migration and maturation, and cryoanesthesia are likely to have a smaller, if any, effect on cortical morphology after adult stroke. Secondary neuronal degeneration and stretching/shifting of residual tissue into the infarct, however, may significantly alter residual cortical morphology in the adult rat.

For the aforementioned reasons, I propose the investigation of a novel "plastic" model of adult rat cortex after focal cortical stroke. This "plastic" model is based on the hypothesis that significant thinning and stretching of residual cortical tissue could occur after focal cortical stroke in the adult rat. A hypothetical illustration of this "plastic" model is shown in figure $2 \mathrm{~B}$, and is contrasted with the traditional "topographical" model shown in figure $2 \mathrm{~A}$.
\end{abstract}



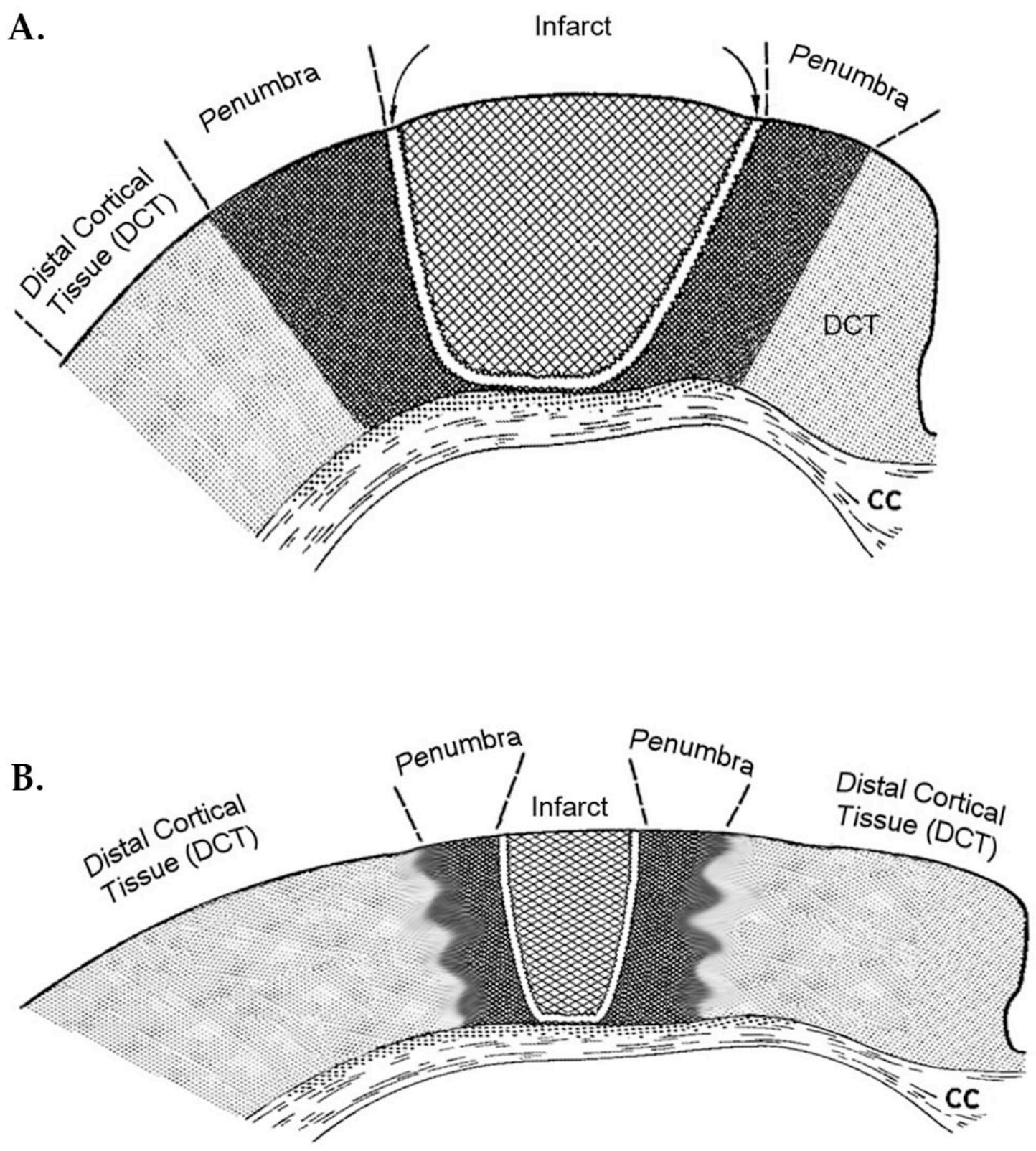
Figure 2. The traditional "topographical" model of residual cortical morphology in the adult rat (A, adapted from Bidmon et al., 1997). The infarct, penumbra, and distal cortical regions are relatively fixed and defined. The new "plastic" model of residual cortical morphology in the adult rat (B). Morphological changes such as cortical thinning and stretching could lead to dynamic changes in the shape and location of the infarct, penumbra, and distal cortical regions. DCT - Distal Cortical Tissue, CC - Corpus Callosum 
Validation of this new "plastic" model requires the systematic investigation of post-stroke morphological change in the adult rat. Such an investigation is crucial for a number of reasons. Firstly, the majority of preclinical stroke research utilizes adult rats to model human stroke. Thus, it is essential to determine the general nature, as well as the temporal and spatial development of morphological change in these animals to ascertain the applicability of these models to the human condition.

Secondly, understanding the morphological state of residual cortical tissue is essential for ensuring the validity of lesion studies for determining the function of a localized region of brain tissue. If localized lesions have widespread morphological effects on distal residual tissue, then the lesion may not really be localized at all.

Thirdly, understanding morphological change after adult stroke is essential for ensuring the validity of experimental investigations of biochemical, cytoarchitectural, and electrophysiological properties of residual peri-infarct tissue. Changes in post-stroke tissue morphology (ie. size, shape, and/or location), if left unaccounted for, could lead to the erroneous comparison of non-homologous brain regions in stroke vs. non-stroke brains. This is especially important when trying to identify neuroplastic substrates in residual tissue that may mediate post-stroke functional recovery.

Finally, the majority of experiments investigating morphological change utilize histological techniques. The preparation of histological tissue, however, introduces morphological anomalies by nature. Tissue perfusion, removal, sectioning, and dehydration can artificially shrink neural tissue, distort anatomical structures, and introduce nicks, cuts, and depressions in otherwise "normal" tissue. Thus, because systematic in vivo investigations of peri-infarct tissue morphology have not been done, 
the absolute changes in post-stroke residual tissue morphology are not known. As a large number of experiments utilize in vivo manipulations to study peri-infarct tissue function, it is important to determine the individual contribution of histological preparation vs. in vivo stroke infarct formation to morphological change in residual cortical tissue.

\section{Objectives of the Present Study}

There are three main aims of this thesis: 1) to document and characterize the temporal and spatial development of morphological change that occurs after forelimb motor cortex stroke in the adult rat; 2) to determine the extent to which post-stroke morphological change varies as a function of stroke induction method, location, and size;

and 3) to determine the influence of histological tissue processing on the interpretation of post-stroke cerebral morphology in the adult rat.

To achieve the first objective, adult rats received unilateral pial strip devascularization stroke of forelimb motor cortex and were culled at consecutive time points between 1 hour and 31 days post-stroke. Gross morphological changes in residual brain were then analyzed from the recovered histological tissue. The pial strip devascularization stroke model was chosen for a number of reasons. Firstly, this type of stroke models many aspects of human focal cortical stroke. Specifically, deficits in skilled reaching behaviour have been described in detail using this model (Whishaw 2000; Whishaw et al., 1986a; 1991) and effectively model skilled forelimb deficits in human stroke. Secondly, this is a focal cortical stroke model for which the infarct size and location are readily reproducible. Finally, the cytoarchitectural, biochemical, and 
electrophysiological changes that occur in residual cortical tissue have been thoroughly described after forelimb motor cortex stroke (Brown et al., 2008; Whishaw et al., 2008). For the purposes of the present study, it was important that the infarct was localized to the cortex and did not extend to subcortical structures as may occur in other stroke models, to ensure that changes in distal residual tissue were not due to indirect degeneration of afferent inputs from the striatum.

To achieve the second objective, cortical thinning was examined in the chronic post-stroke time period in various rodent models of stroke. Firstly, the effect of stroke size was examined by varying the size of the pial strip devascularization forelimb infarct. Secondly, the effect of stroke induction method was examined by employing a second focal cortical stroke model - photothrombosis. Not only does the photothrombotic model produce a comparable stroke infarct to the standard pial stip model but it also allows for the investigation of the effects of open- vs. closed-head surgical manipulations on poststroke cortical morphology. Finally, to examine the effect of stroke location, a middle cerebral artery occlusion (MCAO) model was employed. The extent of damage produced by this method can vary; damage may be localized to subcortical structures or extent to include cortical structures as well. Thus, it was possible to determine the extent to which cortical thinning is a feature of motor cortex stroke, focal cortical stroke, or stroke more generally.

Lastly, to determine the extent to which histological anomalies may influence post-stroke cerebral morphology a final group of animals received pial strip devascularization of forelimb motor cortex and underwent serial magnetic resonance imaging (MRI) from 1 hour to 31 days post-stroke. This allowed us to resolve the 
spatiotemporal development of post-stroke morphological change in vivo. Furthermore, because tissue movement has been suggested as a contributing factor to post-stroke morphological change in the adult rat, this was also examined in vivo. Markers of intact tissue location were placed in the cortex at the time of stroke induction and their change in location, relative to the stroke infarct, was tracked in the serial MRI images.

In the final chapter, the results of the three major comparisons will be evaluated as well as the implications of these findings for the comparison of homologous brain tissue in stroke and non-stroke brains. It concludes by addressing the fundamental aim of the thesis: determining the nature of post-stroke morphological change in the cortex of the adult rat. Finally, I discuss the theoretical implications of employing a new "plastic" model of residual cortical morphology for future investigations of residual tissue function. 


\title{
CHAPTER TWO
}

\section{SPATIOTEMPORAL CHANGES IN HISTOLOGICAL CORTICAL THICKNESS AFTER PIAL STRIP DEVASCULARIZATION STROKE OF THE FORELIMB AREA OF MOTOR CORTEX IN THE ADULT RAT}

\begin{abstract}
Plasticity of residual cortical tissue has been identified as an important mediator of functional post-stroke recovery. Many studies have been directed toward describing biochemical, electrophysiological, and cytoarchitectural changes in residual cortex and correlating them with functional changes. Additionally, after neonatal stroke the thickness of residual tissue can change, the tissue can move, and tissue can fill in the stroke core. The purpose of the present study was to systematically document such gross morphological changes in peri-infarct tissue after forelimb motor cortex stroke in the adult rat. Rats received a forelimb unilateral stroke by pial strip devascularization and were then examined using histology at 1 hour, 1, 3, 7, 14, or 31 days post-stroke. Decreases in cortical thickness, volume, and neural density were found to extend far beyond the stroke infarct and included sensorimotor regions of the intact hemisphere. Thus, extensive time-dependent morphological changes that occur in residual tissue must be considered when evaluating plasticity-related cortical changes associated with poststroke recovery of function.
\end{abstract}




\section{Introduction}

Stroke to the forelimb region of rat motor cortex and the changes in skilled forelimb use that follow have been effectively used as a model of human motor system stroke (Whishaw and Pellis, 1990). Extensive analyses following stroke indicate that behavioral changes in the acute and chronic post-stroke periods are quite different. In the first few days after stroke, skilled movements are extremely impaired and animals learn not to use their affected limb (Erickson et al., 2007; Alaverdashvili et al., 2008).

Thereafter, animals regain skilled action but do so via the use of compensatory strategies (Whishaw et al., 1986a, 1991; Whishaw 2000; Metz et al., 2005; Gharbawie et al., 2007). These acute behavioral changes may be associated with morphological changes in periinfarct and distal cortical tissue, including the dissipation of edema, cytoarchitectural changes, glial scar formation, hypo- and hyperperfusion, gene expression, protein expression, and electrophysiological changes (Schroeter et al., 1995; Bidmon et al., 1998;

Johansson et al., 2000; Kokubo et al., 2003; Kury et al., 2004; Brown et al., 2007, 2008; Brown and Murphy, 2008; Nowicka et al., 2008). In addition, it has been suggested that the compensatory improvements in skilled behavior that occur in the chronic period may be associated with long-lasting plastic changes in residual tissue including changes in neuronal structure, cortical organization, neurotrophic factor regulation, and electrophysiological responsiveness (Eysel and Schweigart, 1999; Schiene et al., 1999; Kolb et al., 2001; Frost et al., 2003; Gonzalez and Kolb, 2003; Reinecke et al., 2003; Fridman et al., 2004; Zepeda et al., 2004). 
One difficulty in analyzing the contributions of residual tissue to recovery is a lack of understanding of how a cortical infarct affects the gross morphology of surrounding cortical tissue. Proximal peri-infarct tissue may become distorted by the infarct, it may change in thickness over time, and it may move toward the infarct, filling in the stroke cavity. Such changes vary, however, as a function of the stroke location, size, and the method of stroke induction. Comparisons between peri-infarct and "normal" tissue function are also complicated by the choice of a control. Some studies make comparisons to tissue in a non-stroke control animal, whereas others use the intact contralateral hemisphere within the same stroke animal. Finally, age may be a contributing factor as stroke in infant subjects produces extensive gross morphological changes in remaining tissue including thinning, movement, and filling in of the stroke infarct (Kolb and Whishaw, 1981; Kolb and Holmes 1983; Kolb et al., 1983a,b, 1987; Kolb 1987; Kolb and Cioe, 2000; Kolb et al., 2000; Whishaw, 2000; Alaverdashvili and Whishaw, 2010). In short, the complexity of these morphological changes makes it difficult to make comparisons between homologous cortical regions in intact control rats and stroke rats. Because there has been no systematic analysis of the gross morphology of peri-infarct cortex following forelimb motor cortex stroke in the adult rat, the present study was directed at characterizing gross peri-infarct morphological changes in the acute and chronic post-stroke period.

The effect of forelimb motor cortex stroke on residual cortical thickness in histological tissue was examined in the adult rat. Animals received standard motor cortex pial strip devascularization stroke $(n=18)$ and were randomized into six different groups for sacrifice at the following post-surgical time points: 1 hour (H), 1 day (D), 3D, 7D, 
$14 \mathrm{D}$, and $31 \mathrm{D},(\mathrm{n}=3$ each $)$. A seventh group of rats $(\mathrm{n}=4)$ received sham motor cortex pial strip devascularization surgery and was also sacrificed at 31D to act as a control group. All brains were collected and processed for cresyl violet staining. Measures of cortical thickness along the coronal (mediolateral) and sagittal (rostrocaudal) plane, and planimetric measures of residual cortical volume were taken from each rat at each time point. In addition, neuronal density was inferred from measures of Grey Value Index (GVI) at 31D.

\section{Methods and Material}

\section{Subjects}

Subjects were male Long-Evans hooded rats, 90 days of age, and weighing 350550g. Rats were housed in groups of two in Plexiglas cages with cob bedding. The colony room in which they were housed was temperature and humidity controlled and maintained on a $12 \mathrm{~h} / 12 \mathrm{~h}$ light/dark cycle. Purina rat chow and water were available ad libidum. All experiments complied with the guidelines of the University of Lethbridge Animal Care Committee and the University of Calgary Animal Care Committee as set out by the Canadian Council for Animal Care.

\section{Pial Strip Devascularization Stroke}


The forelimb region of the motor cortex, as previously defined by behavioral (Whishaw 2000), anatomical (Donoghue and Wise, 1982), and electrophysiological (Hall and Lindhom, 1974; Donoghue and Wise 1982) studies was targeted for devascularization. Animals received an injection of atropine methyl nitrate $(0.1 \mathrm{mg} / \mathrm{kg}$ i.p.; Sigma-Aldrich, St. Louis, MO) to facilitate respiration throughout surgery, burprenorphine $(0.05 \mathrm{mg} / \mathrm{kg}$ s.c.; Schering-Plough, Hertfordshire, UK) as an analgesic, and an injection of sodium pentobarbital (65 mg/kg, i.p.; Sigma-Aldrich, St. Louis, MO) for anaesthetization. A small incision was made in the scalp along the midsagittal suture and a fine dental burr was used to drill four holes in the skull above motor cortex. The coordinates of these holes were measured from Bregma; i) Anterior (A) -1.0 mm, lateral (L) $+1.0 \mathrm{~mm}$; ii) A -1.0 mm, L +4.0 mm; iii) A +4.0 mm, L +4.0 mm; iv) A +4.0 mm, L $+1.0 \mathrm{~mm}$. The rectangular area of skull demarked by the four holes was then removed using a dental burr and the dura mater below the trephination was cut and peeled away. The exposed motor cortex was devascularized by gently wiping away the pia and removing blood vessels with a saline-soaked cotton swab. This procedure does not involve physical removal of the affected tissue. Thus, the stroke cavity remained filled with tissue until the degenerating debris was cleared by glia. The trephination was left uncovered, the incision sutured closed, and the animal's condition monitored in a recovery room for 24 hours. Sham-operated animals were treated similarly except that the dental burr did not perforate the skull, and the processes of trephination and devascularization were not performed.

\section{Histology}


Rats were deeply anesthetized with sodium pentobarbital and intracardially perfused with $0.9 \%$ phosphate-buffered saline (PBS) then fixed with $4 \%$ paraformaldehyde (PFA) in PBS. The brains were removed and post-fixed in 4\% PFA for $24 \mathrm{~h}$ at $4{ }^{\circ} \mathrm{C}$. They were then placed in cryoprotectant (30\% glucose in $4 \%$ PFA) and sectioned on a microtome after sinking. Serial $40 \mu \mathrm{m}$ sections were obtained from the olfactory bulb to the cerebellum, mounted on gel-coated slides, stained with cresyl violet, and cover slipped.

\section{Cortical Thickness}

The method for measuring cortical thickness was adapted from previously published methods (Kolb and Whishaw, 1981; Kolb and Cioe, 2001). A summary of the measurement procedure, using 6 representative histology sections is illustrated in Figure 3. Ten tissue sections, located at $1 \mathrm{~mm}$ intervals, were examined. For each tissue section, Image J software (v. 1.38X) was used to take up to twenty measurements of cortical thickness from each coronal section, ten from each hemisphere. Measurement 1 was taken medial to the infarct, measurement 2 was taken lateral to the infarct and all successive measurements were taken lateral to the previous measurement. In this manner, the infarct was always located between measurements 1 and 2, and only intact tissue was ever measured in the stroke hemisphere. Two additional measurements were taken in the forelimb area of motor cortex in the five most rostral sections of the intact and control 


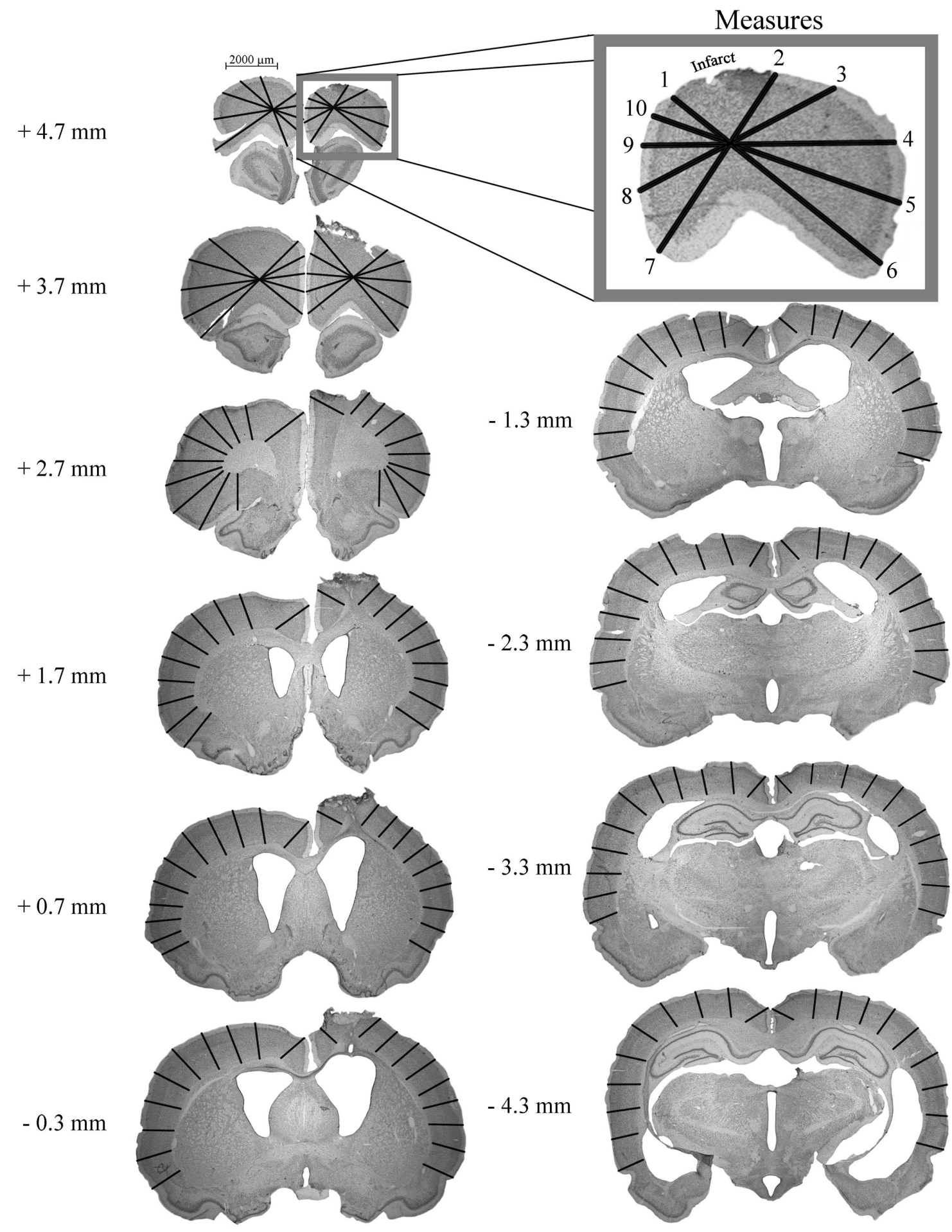


Fig. 3. Planes of measurement illustrated in a pial strip stroke rat stained with cresyl violet. Plane 1 is the most rostral point of measurement and Plane 10 is the most caudal point of measurement. The 10 mediolateral vectors from which cortical thickness was measured is illustrated on plane 1 (in the box). At plane 1 and 2, a diagonal line was drawn from immediately medial to the stroke core to the opposite edge of the cortex. A second diagonal line was drawn from immediately lateral to the stroke core to the opposite edge of the cortex creating an X. Additional vectors of measurement were taken from the center of this $\mathrm{X}$ out to the edge of the cortex. For planes 3 through 10 each measurement was made from the cortical surface to the dorsal surface of the corpus callosum at an orientation that was a tangent to the surface of the corpus callosum. Note: measurements were not made in the infarct in the stroke hemisphere. 
hemispheres. However, only measures outside of the forelimb area of motor cortex were compared across all three groups. For tissue sections 1 and 2, measurements were made along a vector from the center of the hemisphere, out to the edge of the cortex. For all other tissue sections, measurements were made along a vector from the tangent of the outer edge of the corpus callosum to the edge of the cortex. Analyses of cortical thickness along the coronal plane were obtained by grouping all rostrocaudal sections together and making statistical comparisons across all mediolateral measures. Likewise, analyses of cortical thickness along the sagittal plane were obtained by grouping all mediolateral measures within each coronal section together and making statistical comparisons across all rostrocaudal sections.

\section{Cortical Volume}

Measures of cortical volume were taken from the same 10 tissue sections that cortical thickness measures were taken from. Residual cortical tissue was visually distinguished from the adjacent corpus callosum and stroke core. In each hemisphere, the area defined as residual cortex was then outlined and the enclosed area $\left(\mathrm{mm}^{2}\right)$ calculated using Image J software (v. 1.38X). The volume of remaining cortical tissue was calculated by the following formula, where "slice thickness" refers to the $1 \mathrm{~mm}$ distance between tissue sections:

Total residual cortex volume $\left(\mathrm{mm}^{3}\right)=$ the sum of the areas $\left(\mathrm{mm}^{2}\right)$ x slice thickness $(\mathrm{mm})$. 


\section{Neural Density}

Neural density was inferred from measures of Grey value index (GVI) (Zilles et al., 1980; Moon et al., 2009). GVI was analyzed in tissue medial and lateral to the midpoint of the infarct $(+1.7 \mathrm{~mm}$ from bregma) from rats that were survived for 31 days post pial strip stroke. Photographs of Nissl-stained tissue were converted to 32-bit grey scale images and GVI was analyzed using Image J software (v. 1.38X). GVI was measured across the spared cortex along 10 vectors tangent from the outer edge of the corpus callosum to the edge of the cortex (the same 10 vectors that were used to measure cortical thickness, Figure 2). Measures of GVI were normalized relative to the GVI of the ipsilateral lateral ventricle.

\section{Statistical Analysis}

Results were subject to analysis of variance (ANOVA) and follow-up LSD tests with the computer program SPSS (v.16.0). A p-value of less than 0.05 was considered significant. TIME (1H, 1D, 3D, 7D, 14D, and 31D) and HEMISPHERE (control vs. intact vs. stroke) served as between-subjects factors. ROSTROCAUDAL SECTION (1, 2, 3, 4, 5, 6, 7, 8, 9, and 10) and MEDIOLATERAL MEASURE (1, 2, 3, 4, 5, 6, 7, 8, 9, and 10) served as within-subject factors.

\section{Results}




\section{Residual Cortical Thickness: Temporal Changes}

Figure 4 summarizes the change in histological cortical thickness over 31 poststroke days after pial strip forelimb motor cortex lesions.

Figure 4A gives line tracings of a representative coronal section through the midpoint of the infarct from a 3D animal (left, gray area) and a 31D animal (right, gray

area). The surrounding outline on both sections is that of a control section from a nonstroke animal. It is noteworthy that that the infarct is well developed in the 3D animal and the residual cortex in the $31 \mathrm{D}$ animal is relatively thinner than that of the $3 \mathrm{D}$ animal.

The detailed measurements from the stroke hemisphere and the intact hemisphere were made from tissue from each of the designated post stroke days (1H to 31D). The results represent a mean of all measures on all of the sections (see methods). In addition the results were subdivided into an acute group composed of the $1 \mathrm{H}$ to $3 \mathrm{D}$ animals and a chronic group composed of the 7D to $31 \mathrm{D}$ animals. Figure 4B shows that tissue in both the intact and the stroke hemisphere became thinner over time, and that thinning was more pronounced in the stroke hemisphere and in the chronic period.

Figure $4 \mathrm{C}$ shows the average thickness in the intact hemisphere vs. the stroke hemisphere in the acute and chronic time periods. While both the intact and lesion hemispheres sustained significant thinning of residual cortical tissue in the chronic time period, the loss in the lesion hemisphere was greater. Ultimately, the intact hemisphere sustained an approximate $7.5 \%$ loss in cortical thickness outside of the homotopic forelimb motor cortex, while the stroke hemisphere sustained an approximate $18.5 \%$ loss in residual cortical tissue outside of the stroke infarct. In summary, despite the stroke 


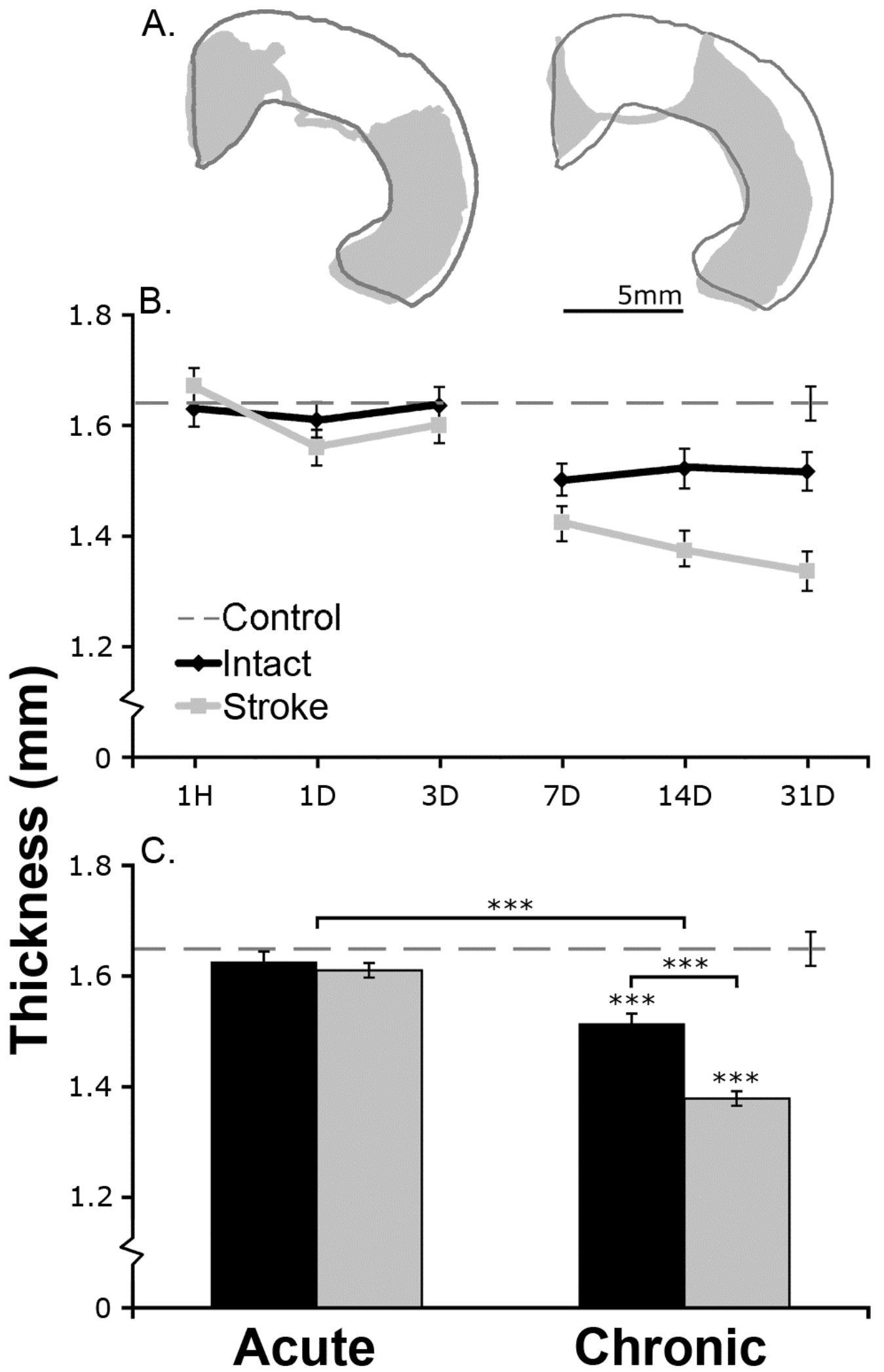


Fig. 4. Cortical thickness (mean and standard error) summed over sagittal and coronal measures at different post-stroke acute $(1 \mathrm{H}-3 \mathrm{D})$ and chronic $(7 \mathrm{D}-31 \mathrm{D})$ times. A. Illustration of the cortex (shaded area) of a pial strip rat at 3D (left) and 31D relative to a control section (outline). B. Cortical thickness in the non-stroke control group (dotted line, $n=4$ ), the intact hemisphere of the stroke groups (black, $n=3$ per time point), and the stroke hemisphere of the stroke groups (gray, $n=3$ per time point) at acute and chronic time points. C. Mean cortical thickness in the acute period vs. chronic period. Note: cortical thinning is progressive in both hemispheres of the stroke groups with the stroke hemisphere most affected in the chronic period, (H - Hour, D - Day, ***p $<$ $0.001)$. 
infarct being largely developed by 3D post-stroke, the majority of cortical thinning occurs in the chronic (7D to 31D) time period and in the stroke hemisphere. This suggests that cortical thinning does not temporally parallel tissue degeneration and clearance in the stroke infarct. Rather, thinning of residual cortical tissue is a time-dependent phenomenon that largely occurs after the formation of the stroke infarct.

These main findings were confirmed by the statistical analysis. There was a significant effect of $\operatorname{TIME}[\mathrm{F}(5,27)=18.111, \mathrm{p}<0.001]$, HEMISPHERE $[\mathrm{F}(1,27)=$ 16.139, $\mathrm{P}<0.001]$, and TIME X HEMISPHERE $[\mathrm{F}(5,27)=3.092, \mathrm{P}<0.05]$. The post-hoc analyses confirmed that the cortical thinning was greatest in the chronic period and more pronounced in the stroke vs. the intact hemisphere $(\mathrm{p}<0.001)$.

\section{Coronal and Sagittal Cortical Thickness}

Figure 5 gives a summary of cortical thickness from all of the groups from which measures were obtained in the chronic period (7D to 31D).

Figure 5A shows measures of cortical thickness from 10 mediolateral coronal locations summarized across 10 rostrocaudal sections. The first measurement location was taken medial to the infarct while the remaining measurement locations were taken lateral to the infarct (gray bar). The cortex was thinner in stroke animals than in the control animals and the stroke hemisphere was thinner than the intact hemisphere in the stroke group. Figure 5B illustrates the change in residual cortical thickness from just posterior of the olfactory bulb back to the most caudal section measured, $4.3 \mathrm{~mm}$ posterior to bregma (sagittal axis). Along this sagittal axis, cortical thinning was again greatest in 


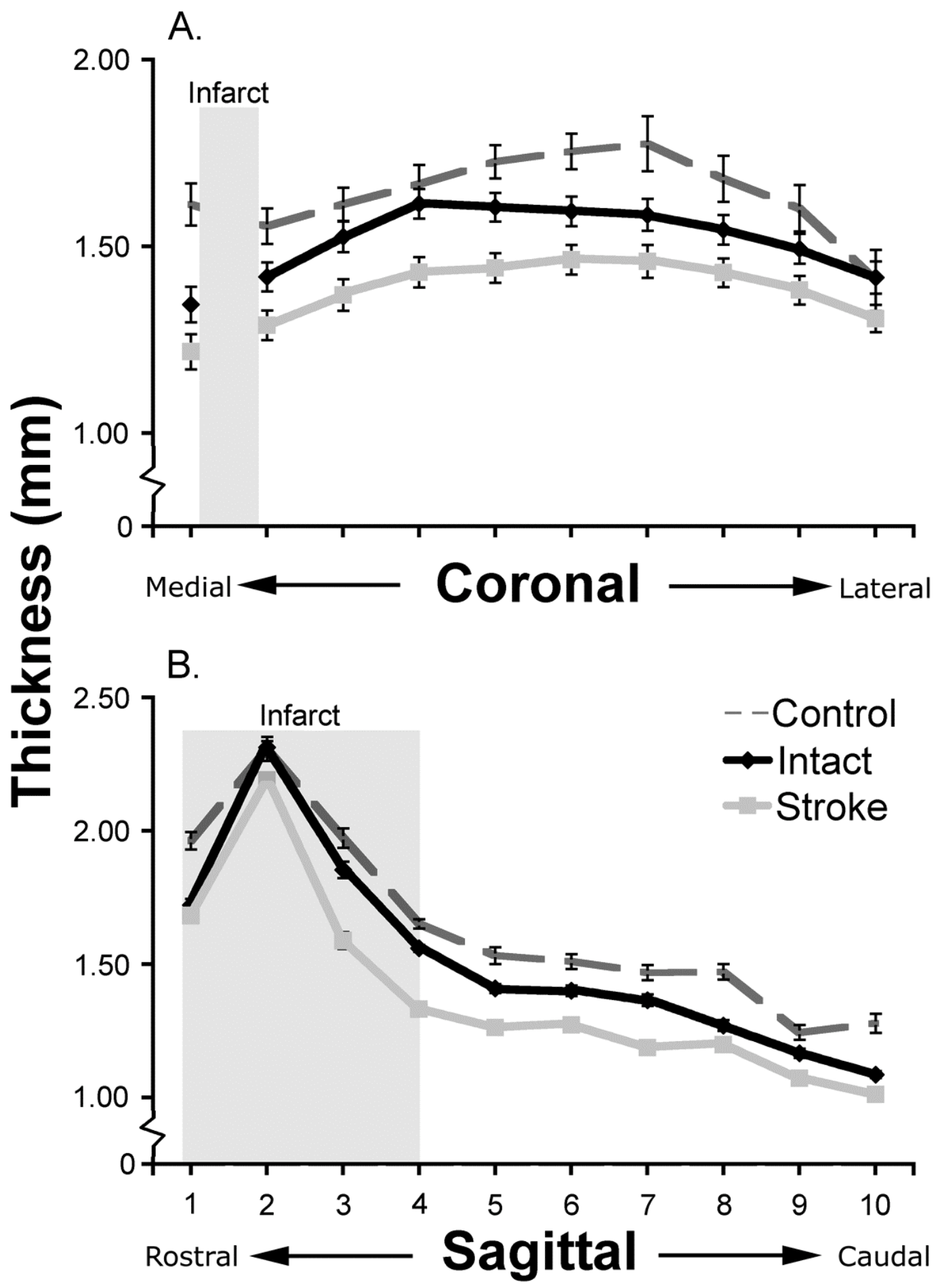


Fig. 5. Cortical thickness (mean and standard error) of coronal (A) and sagittal (B) points of measurement from non-stroke control rats (dotted line, $n=4)$, the intact hemisphere of stroke rats (black, $n=9$ ), and the stroke hemisphere of stroke rats (gray, $n=9$ ) in the chronic time period. Note: Reductions in cortical thickness were greatest proximal to the stroke infarct but also extended to distal cortical tissue. 
the stroke hemisphere, but also occurred in the intact hemisphere. Thinning was greatest proximal and immediately caudal to the infarct, but extended to distal residual tissue as well. In summary, this finding suggests that proximal peri-infarct tissue is more susceptible to morphological change after stroke (ie. cortical thinning), but that these changes can also extend to distal functional areas of remaining cortex.

The statistical analysis confirmed that the cortex was thinner in the stroke groups than in the control non-stroke group and that the stroke hemisphere was thinner than the intact hemisphere within the stroke groups, HEMISPHERE X MEASURE $[\mathrm{F}(18,171)=$ 4.524, $\mathrm{p}<0.001]$ and HEMISPHERE X SECTION $[\mathrm{F}(18,171)=2.164, \mathrm{p}<0.01]$.

\section{Temporal Measures of Cortical Thickness in the Stroke Hemisphere}

Figure 6 shows cortical thickness in the stroke hemisphere at each of the time points from which measurements were taken.

Figure 6A gives measures of cortical thickness from 10 mediolateral coronal locations averaged across 10 rostrocaudal sections at each post-stroke time point. The cortex was systematically thinner at successively later time points. Figure 6B gives measure of cortical thickness from 10 rostocaudal sections averaged across 10 mediolateral coronal measures. The cortex was systematically thinner at successively later time points. Cortical thickness losses between $1 \mathrm{H}$ and $31 \mathrm{D}$ ranged from $13.9 \%$ to $26.9 \%$ along the coronal axis. Cortical thickness losses along the sagittal axis ranged from $13.1 \%$ to $31.7 \%$. Again, it is noteworthy that the greatest reduction in cortical thickness occurs proximal to the stroke infarct. However, it is interesting that thickness 


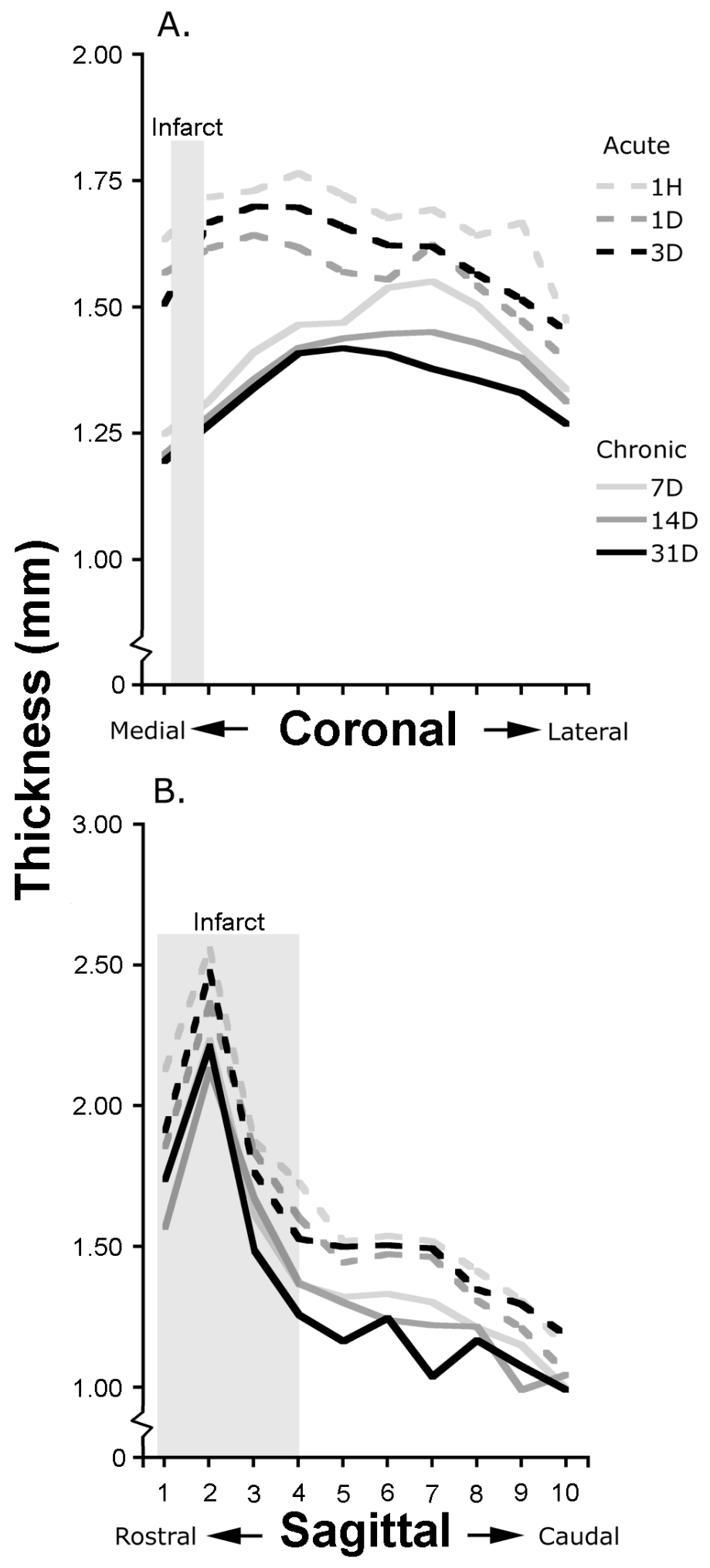


Fig. 6. Cortical thickness (mean) in the stroke hemisphere at coronal (A) and sagittal (B) points of measurement from groups of rats with pial strip strokes ( $n=3$ per group). Note: Reductions in cortical thickness developed gradually over time in most instances. However, residual tissue just medial to the infarct sustained a significant decrease in thickness between 3D and 7D post-stroke (H - Hour, D - Day, Dashed lines - acute period, Solid lines - chronic period, shading represents planes containing the infarct). 
loss along the coronal axis varies with location. Proximal peri-infarct tissue shows a large decrease in cortical thickness between 3D and 7D, whereas thickness losses in more distal peri-infarct tissue are more gradual. It may be possible that different mechanisms of cortical thinning occur proximal vs. distal to the stroke infarct. Losses in cortical thickness along the sagittal axis also appear to develop gradually.

The results were confirmed by ANOVA's that gave significant effects of TIME X $\operatorname{MEASURE}[\mathrm{F}(45,108)=2.311, \mathrm{p}<0.001]$ and TIME X SECTION $[\mathrm{F}(45,108)=1.542$, $\mathrm{p}<0.05]$.

Figure 7 summarizes the reduction in cortical thickness in the stroke and intact hemispheres as a percent difference from the cortical thickness in the control group. In general, the greatest reduction in cortical thickness occurred near the stroke core but thinning did occur at the most distal measured locations and in homotopic points in the intact hemisphere.

\section{Cortical volume}

Figure 8 shows the cortical volume, summarized across 10 rostrocaudal sections, over 31 post-stroke days after pial strip forelimb motor cortex stroke.

Figure $8 \mathrm{~A}$ shows that cortical volume was smaller in the stroke groups than in the control group and cortical volume was smaller in the stroke hemisphere than in the intact hemisphere. In addition cortical volume was smaller in the chronic groups than in the acute groups. Figure $8 \mathrm{~B}$ shows the average cortical volume in the intact hemisphere vs. the stroke hemisphere in the acute and chronic time periods. While both the intact and 


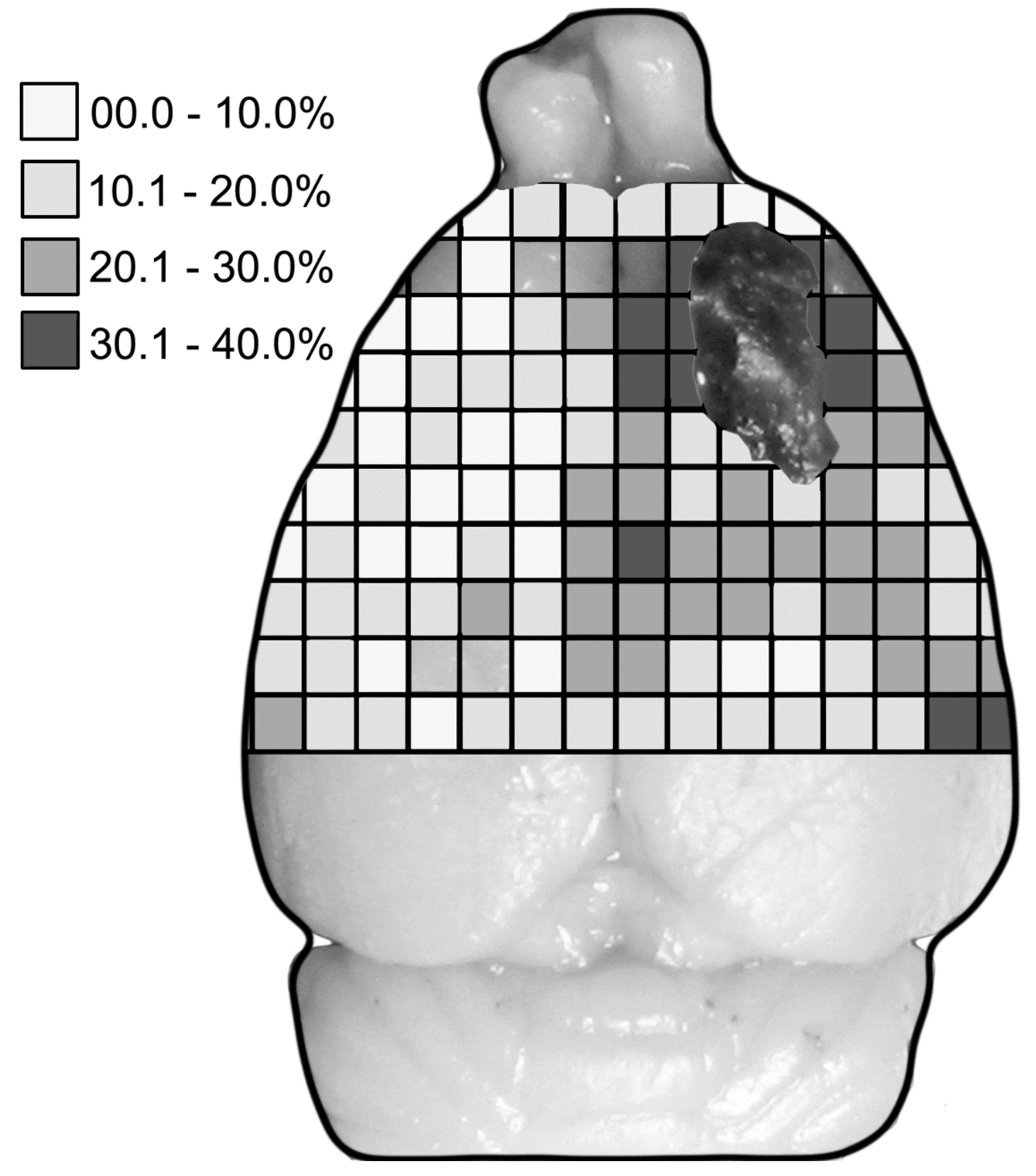


Fig. 7. A summary illustration of cortical thickness change (percent of control) in the chronic period in the stroke hemisphere (right, $\mathrm{n}=9$ ) and the intact hemisphere (left, $\mathrm{n}=$ 9) of pial strip devascularization rats. A representative infarct is shown in the outlined area of the cortex. Note: cortical thinning was generally greater in the stroke hemisphere, proximal to the stroke infarct. However, thinning also occurred in the intact hemisphere. 


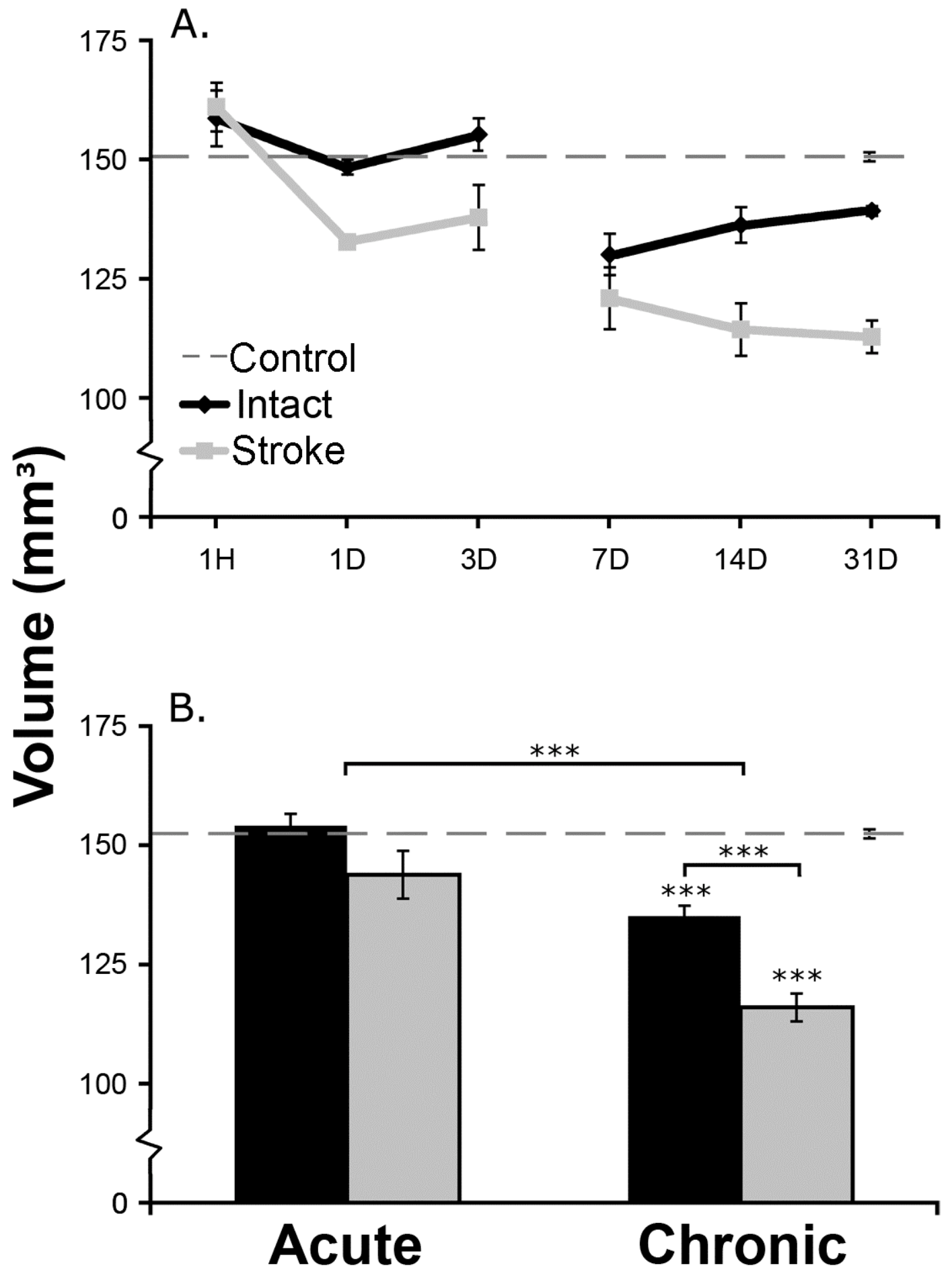


Fig. 8. Cortical volume (mean and standard error) summed over sagittal and coronal measures at different post-stroke acute $(1 \mathrm{H}-3 \mathrm{D})$ and chronic $(7 \mathrm{D}-31 \mathrm{D})$ times. A. Cortical thickness in the non-stroke control group (dotted line, $n=4$ ), the intact hemisphere of the stroke groups (black, $n=3$ per time point), and the stroke hemisphere of the stroke groups (gray, $\mathrm{n}=3$ per time point) at acute and chronic time points. B. Mean cortical thickness in the acute period vs. chronic period. Note: cortical volume loss is progressive in both hemispheres of the stroke groups with the stroke hemisphere most affected in the chronic period, (H - Hour, D - Day, $\left.{ }^{* * *} \mathrm{p}<0.001\right)$. 
stroke hemisphere had reduced cortical volume in the chronic time period, the loss in the stroke hemisphere was greater. Cortical volume decreased significantly in the stroke hemisphere by 1D. It is likely that this initial volume loss is due to tissue loss within the stroke infarct. It is interesting that subsequent volume loss mirrors cortical thickness loss, in that the greatest reductions occur in the chronic time period (7D-31D). It is possible that the two are causally linked.

These main findings were confirmed by ANOVA's that gave significant effects of TIME $[F(1,27)=36.576, p<0.001]$, and TIME X HEMISPHERE $[F(5,27)=2.932$, $\mathrm{p}<0.05]$.

\section{Cortical Tissue Density}

Figure 9 shows measures of cortical tissue density at 10 mediolateral coronal locations summarized across 10 rostrocaudal sections that encompassed the lesion core from 31D pial strip animals. Tissue density was greater in the stroke hemisphere in measures immediately medial and lateral to the infarct (gray bar). Tissue density in the stroke hemisphere was reduced in measures taken from more distal and lateral locations. The fact that tissue density is increased proximal to the infarct but decreases at distal locations is indicative of two things; firstly, tissue density proximal to the stroke core is likely increased due to the infiltration of astrocytes and formation of a glial scar; secondly, despite thinning of distal residual tissue, neuropile density is decreased. This suggests that thinning of residual tissue is not simply due to flattening of residual tissue, 
which would increase tissue density, but rather that neuropile loss is a feature of thinned residual post-stroke tissue.

These main findings were confirmed by an ANOVA that gave no significant effect of HEMISPHERE $[F(1,2)=2.930, p>0.05]$ but a significant effect of HEMISPHERE X MEASURE $[\mathrm{F}(9,18)=5.486, \mathrm{p}<0.001]$.

\section{Discussion}

The effect of forelimb motor cortex pial strip devascularization stroke on the gross morphology of surrounding sensorimotor cortical tissue was measured in the adult rat during the acute (1H to 3D) and chronic (7D to 31D) post-stroke periods. Changes in cortical thickness, volume, and density in the stroke, intact, and non-stroke control hemispheres were dependent variables. Decreases in cortical thickness, volume, and tissue density were found to extend far beyond the infarct core in the chronic period. These results indicate that gross morphological change does occur in residual cortical tissue after forelimb motor cortex stroke in the adult rat. These changes may provide

insight into behavioral changes post-stroke and should prove useful in investigating periinfarct tissue and its function after stroke.

Extensive and progressive gross morphological change, as measured by cortical thickness, volume, and density occurred in peri-infarct tissue after cortical stroke. Although the stroke infarct was well defined by 3D post-stroke, the largest decreases in residual cortical thickness occurred after 3D post-stroke. This result indicates that 


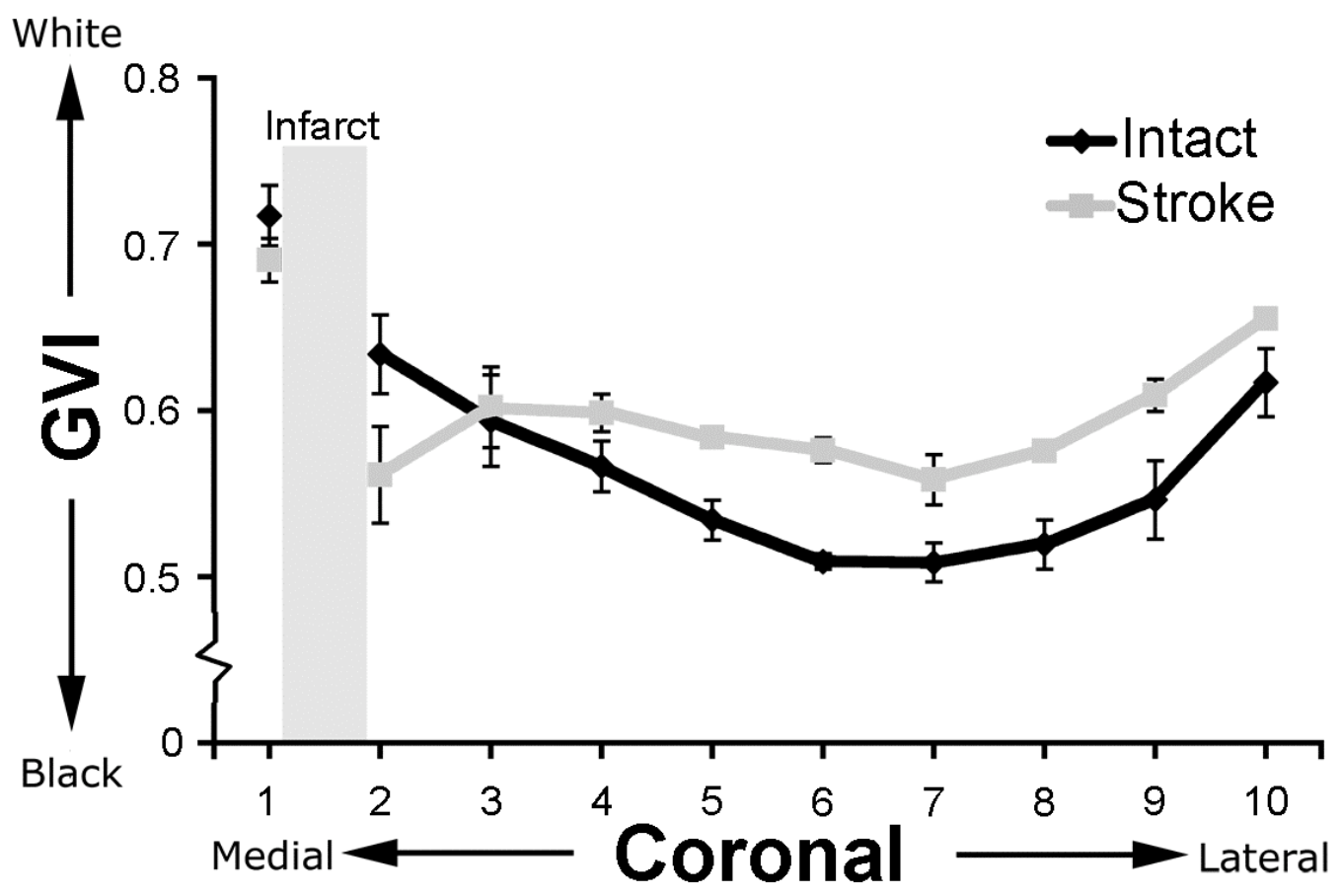


Fig. 9. Cortical tissue density (mean and standard error) summed over 10 cresyl violet coronal sections encompassing the infarct in $31 \mathrm{D}$ pial strip rats. The intact hemisphere of the stroke group (black, $n=3$ ) and the stroke hemisphere of the stroke group (gray, $n=3$ ) are shown. A lower GVI indicates greater tissue density thus indicating a loss of neuropile in the stroke hemisphere. Note: tissue density increased proximal to the stroke infarct, but decreased throughout the remaining residual cortex (GVI - Gray Value Index; D - Day; shading represents planes containing the infarct). 
thinning of residual cortex does not temporally parallel tissue degeneration and clearance in the stroke infarct. Rather, residual tissue thinning occurs subsequent to infarct formation. Whether or not the formation of an infarct is necessary for subsequent cortical thinning is not yet certain. Interestingly, tissue volume loss did mirror changes in tissue thickness, in that the greatest losses in tissue volume occurred, not immediately after stroke induction/formation but also in the chronic post-stroke time period. Because substantial volume loss and tissue thinning both occur after formation of the stroke infarct, and appear to follow a similar temporal development, it is possible that the two are causally linked.

Morphological changes were greatest in sensorimotor cortex adjacent to the forelimb stroke infarct and diminished toward more distal portions of sensorimotor cortex. This spatial pattern of cortical thinning in the stroke hemisphere was mirrored to a lesser degree in the intact hemisphere. It is possible that transhemispheric degeneration could contribute to the thickness loss observed in the intact hemisphere. Despite thinning, tissue density was found to increase proximal to the infarct, likely due to astrocyte infiltration and formation of a glial scar (Frontczak-Baniewicz \& Walski, 2006), but decreased in distal and lateral cortical regions. This decrease in tissue density suggests that post-stroke cortical thinning is not simply due to flattening of intact residual tissue, as this would lead to an increase in tissue density, but rather, supports the notion that tissue loss accompanies thinning of residual cortical tissue after stroke.

The rate of residual post-stroke thinning varied as a function of tissue proximity to the stroke infarct. The thickness of proximal peri-infarct tissue was fairly stable during 
the acute (1H to 3D) period, a large decrease occurred between $3 \mathrm{D}$ and $7 \mathrm{D}$, and then again stabilized between 7D and 31D (Fig. 6.) Cortical thinning in more distal cortical tissue was much more gradual. Thus, different mechanisms of cortical thinning may operate in proximal and distal cortical tissue.

Progressive changes in tissue thickness, volume, and density are likely related to the progressive development of the stroke infarct and subsequent secondary neuronal degeneration. It is known that secondary degeneration of efferent axons and abnormal activity of functionally and anatomically related neuronal tissue can occur long after the completion of infarct formation (Bidmon et al., 1998; Buchkremer-Ratzmann et al., 1996; Buchkremer-Ratzmann \& Witte, 1997; Xu et al., 2006). Because the model used in this study was of forelimb motor cortex stroke, which damages tissue with functional connections to adjacent sensorimotor cortex, it is possible that the large decrease in cortical thickness in peri-infarct sensorimotor cortex could be due to the secondary degeneration of functional and anatomical connections, as well as cell loss, in this adjacent cortical region.

A focal point of the present study was a comparison of gross morphological changes in the acute vs. the chronic period. The acute vs. chronic differences are relevant to the behavioral changes associated with recovery from cortical stroke. Animals display a number of regressive acute motor deficits after stroke, notably, they display "learned non-use" in which they learn not to use an affected limb (Erickson et al., 2007; Taub et al., 2006). The chronic deficits are likely associated with acute pathological effects of the stroke. With constraint-induced rehabilitation, animals then show recovery of limb use over the chronic period, during which they learn to use compensatory motor behaviors to 
regain use of the limb. It is interesting that long-term behavioral treatments that improve functional recovery after stroke, such as environmental enrichment and skilled motor training, are most effective in the chronic period and likely capitalize on the widespread neuronal changes that take place in the chronic period (Bury et al., 2000; Chu and Jones, 2000; Comeau et al., 2008). As gross morphological changes were found to be timedependent, it would be informative to extend investigation of such changes beyond the 30 day time point in future studies.

In summary, because plastic properties of peri-infarct tissue have been identified as a critical mediator of post-stroke recovery, a large amount of research effort has been directed toward describing the molecular, cytoarchitectural, and electrophysiological changes that occur in this tissue that could be supporting recovery. The present study is the first to our knowledge to describe such gross morphological changes as thinning, volume loss, and density loss, in residual cortical tissue after stroke in the adult rat. Because these morphological changes have not been considered in previous studies it is uncertain how they may be affecting other investigations of peri-infarct tissue. Thus, future investigations of peri-infarct tissue function must account for residual tissue thinning, volume, and density loss when comparing tissue between stroke and non-stroke animals. 


\title{
CHAPTER THREE
}

\section{CHRONIC CHANGES IN HISTOLOGICAL CORTICAL THICKNESS IN COMMON RODENT STROKE MODELS OF VARYING INDUCTION METHOD, SIZE, AND LOCATION}

\begin{abstract}
Residual cortical thickness, volume, and density loss, both proximal and distal to the stroke infarct, have been described after focal pial strip devascularization of motor cortex in the adult rat. These morphological changes may compromise the interpretation of periinfarct tissue function, which is thought to mediate post-stroke behavioural recovery and compensation. Various rodent stroke models are used in preclinical studies however; thus, the extent to which these morphological changes are a specific feature of pial strip motor cortex stroke, or a more general feature of brain injury is not known. The purpose of the present study was to characterize the extent of cortical thinning that occurs in common rodent models of stroke. Animals received unilateral stroke via pial strip devascularization (standard vs. small size), photothrombotic occlusion, or middle cerebral artery occlusion damaging only subcortical structures (MCAO-S) or damaging both cortical and subcortical structures (MCAO-C). Measures of cortical thickness were taken from residual cortical tissue on post-stroke day 31. Despite variations in stroke induction method, size, and location, thinning of residual cortical tissue occurred in all stroke models provided that a cortical infarct developed. MCAO stroke localized to the basal ganglia did not lead to thinning of the overlying cortex. The results are discussed in
\end{abstract}


relation to the idea that the space created by a cortical infarct may be an important determinant of the extent of thinning of peri-infact tissue.

\section{Introduction}

Numerous rodent models are successfully used to investigate stroke-induced deficits in skilled limb use. Pial strip devascularization and photothrombotic occlusion of motor cortex are utilized in studies aimed at determining the function of a localized area of cortex, as the infarct size, shape, and location created with these models is highly reproducible (Alaverdashvili et al., 2008; Kleim et al., 2007). A major difference between these models is that the pial strip model is an open-head model, whereas the photothrombotic model is a closed-head model. Thus, the effects of trephination on stroke infarct formation may also be studied by comparing the two. Occlusion of the middle cerebral artery (MCAO) represents a large proportion of clinical stroke cases and thus, is widely used in preclinical research (Durukan and Tatlisumak, 2009). The size and location of the stroke infarct in the experimental MCAO model however, is determined by the amount of time that the MCA is occluded. Shorter occlusion times damage only subcortical structures, whereas longer occlusion times produce both cortical and subcortical damage (Kawamura et al., 1991; Mhairi Macrae, 1992). Although this model mimics many aspects of the human stroke condition, it is not always used in preclinical stroke studies because the size of the infarct can be quite variable between animals; the suture insertion procedure is very invasive and can inadvertently damage the external and internal carotid arteries; and, the survival rates of animals undergoing MCAO is 
comparably low, often with less than half of the subjects recovering from surgery. Nevertheless, all of the aforementioned models are commonly used to model human stroke in the rat.

Gross morphological change has been shown to occur in residual tissue after pial strip devascularization of the forelimb area of motor cortex in the adult rat (Karl et al., 2010). These changes included thinning of residual tissue along a spatiotemporal gradient with the greatest amount of thinning occurring proximal to the stroke infarct and in the chronic (7D to 31D) post-stroke time period. Because numerous rodent models are used in preclinical stroke studies, it is important to determine whether the morphological changes observed after pial strip stroke are unique to that model, or whether they are a more general feature of rodent stroke. The purpose of the present study was to determine the extent to which residual cortical tissue thinning is a feature of motor cortex stroke specifically, cortical stroke more generally, or any type of brain damage.

The effect of stroke size, location, and induction method on residual cortical thickness was examined in four different rodent models of adult stroke. Histological tissue collected at 31D from rats that received standard motor cortex pial strip devascularization stroke used in Chapter two was again examined in the present study (pial, $\mathrm{n}=3$ ); another group of animals received small motor cortex pial strip devascularization stroke (small pial, $\mathrm{n}=12$ ); another group received standard motor cortex photothrombotic occlusion stroke (photo, $\mathrm{n}=6$ ); and a final group received middle cerebral artery occlusion stroke (MCAO). From this group, animals were separated into two groups based on post-experimental examination of histological tissue which indicated that some animals sustained large MCAO stroke, in which both cortical and 
subcortical structures were damaged (MCAO-C, $\mathrm{n}=7$ ); while others sustained only subcortical damage (MCAO-S, $n=5$ ). Changes in residual cortical thickness, along the coronal (mediolateral) and sagittal (rostrocaudal) axes were examined from histological tissue.

\section{Methods and Materials}

\section{Subjects}

Subjects were male Long-Evans hooded rats, 90 days of age, and weighing 350550g. Rats were housed in groups of two in Plexiglas cages with cob bedding. The colony room in which they were housed was temperature and humidity controlled and maintained on a $12 \mathrm{~h} / 12 \mathrm{~h}$ light/dark cycle. Purina rat chow and water were available ad libidum. All experiments complied with the guidelines of the University of Lethbridge Animal Care Committee as set out by the Canadian Council for Animal Care.

\section{Standard Pial Strip Devascularization Stroke (MC)}

The forelimb region of the motor cortex, as previously defined by behavioral (Whishaw 2000), anatomical (Donoghue and Wise, 1982), and electrophysiological (Hall and Lindhom, 1974; Donoghue and Wise 1982) studies was targeted for devascularization. Animals received an injection of atropine methyl nitrate $(0.1 \mathrm{mg} / \mathrm{kg}$ i.p.; Sigma-Aldrich, St. Louis, MO) to facilitate respiration throughout surgery, 
burprenorphine ( $0.05 \mathrm{mg} / \mathrm{kg}$ s.c.; Schering-Plough, Hertfordshire, UK) as an analgesic, and an injection of sodium pentobarbital (65 mg/kg, i.p.; Sigma-Aldrich, St. Louis, MO) for anaesthetization. A small incision was made in the scalp along the midsagittal suture and a fine dental burr was used to drill four holes in the skull above motor cortex. The coordinates of these holes were measured from Bregma; i) Anterior (A) - $-1.0 \mathrm{~mm}$, lateral (L) $+1.0 \mathrm{~mm}$; ii) A -1.0 mm, L +4.0 mm; iii) A +4.0 mm, L +4.0 mm; iv) A +4.0 mm, L

$+1.0 \mathrm{~mm}$. The rectangular area of skull demarked by the four holes was then removed using a dental burr and the dura mater below the trephination was cut and peeled away. The exposed motor cortex was devascularized by gently wiping away the pia and removing blood vessels with a saline-soaked cotton swab. This procedure does not involve physical removal of the affected tissue. Thus, the stroke cavity remained filled with tissue until the degenerating debris was cleared by glia. The trephination was left uncovered, the incision sutured closed, and the animal's condition monitored in a recovery room for 24 hours. Sham-operated animals were treated similarly except that the dental burr did not perforate the skull, and the processes of trephination and devascularization were not performed.

\section{Small Pial Strip Devascularization Stroke (sMC)}

Small pial strip devascularization stroke was performed using the same methods as described above except that only the rostral half of the forelimb area of motor cortex was targeted for ischemia. The coordinates of the four drill holes used to demark the area 
of trephination were: i) A $+0.5 \mathrm{~mm}, \mathrm{~L}+2 \mathrm{~mm}$; ii) $\mathrm{A}+0.5 \mathrm{~mm}, \mathrm{~L}+3.5 \mathrm{~mm}$; iii) $\mathrm{A}+2 \mathrm{~mm}$, $\mathrm{L}+3.5 \mathrm{~mm}$; iv) $\mathrm{A}+2 \mathrm{~mm}, \mathrm{~L}+2 \mathrm{~mm}$.

\section{Photothrombotic Occlusion Stroke (PT)}

The method described by Watson and colleagues (1985) was used to induce photothrombotic stroke in 6 animals. Briefly, a Rose Bengal solution is photoactivated in small cortical vessels (diameter $<50 \mu \mathrm{m}$ ) concentrated mainly at the pial surface. This leads to peroxidation of membrane lipids and vascular endothelial damage, which facilitate platelet adhesion and aggregation to the point of vascular occlusion (ie. photothrombosis).

Animals were anesthetized as described for the pial strip forelimb motor cortex devascularization surgery. An incision along the midline was made, the scalp displaced laterally, and the underlying skull exposed. The center of a fiber-optic bundle $(5 \mathrm{~mm}$ diameter) connected to a light source (150-W light bulb) was positioned on the skull $1.5 \mathrm{~mm}$ anterior to bregma and $2.5 \mathrm{~mm}$ lateral to midline (Paxinos and Watson 1998). A rectangular ( $5 \mathrm{~mm}$ X $3 \mathrm{~mm}$ ) aluminum foil stencil was used to give the light source a rectangular shape and to make the photothrombotic lesions comparable in size and shape to the devascularization lesions (Alaverdashvili et al., 2008). The Rose Bengal solution $(10 \mathrm{mg} / \mathrm{kg}$, Sigma-Aldrich, Oakville, ON) was injected via tail vein catheter (Surflo i.v. catheter, Terumo, Tokyo, Japan) within 2 min. After this, the light was turned on and the skull illuminated at an intensity of 170,000 lux for $20 \mathrm{~min}$. The light intensity was selected according to results from previous studies utilizing triphenyltetrazolium chloride 
(TTC) staining 2 days after stroke induction (Alaverdashvili et al., 2008). Rectal temperature was maintained at $37 \pm 1^{\circ} \mathrm{C}$, using a homeothermic blanket system (www.harvardapparatus.com). Following photostimulation, the tail vein catheter was removed and the incision was closed. Sham-operated animals were treated similarly but the light was not turned on $(n=2)$ or no Rose Bengal solution was injected $(n=2)$.

\section{Intraluminal Suture Middle Cerebral Artery-Occlusion (MCAO) Stroke}

The method described by Longa and colleagues (1989) was used to induce transient MCAO stroke in 12 animals. Six rats were intubated under 3-4\% halothane in a mixture of $30 \% \mathrm{O}_{2}$ and $70 \% \mathrm{~N}_{2} \mathrm{O}$. Those rats were maintained on a ventilator for the duration of the procedure, and halothane levels were adjusted to $1 \%$. The ventral tail artery was cannulated in those 6 rats with polythene tubing connected to a Statham transducer. Blood pressure was automatically recorded by a PC computer running labtech software v. 12. Blood samples were drawn throughout the surgery for $\mathrm{pH}$ and gas analyses (Zhu \& Auer, 1995). The rest of the rats were anesthetized in a chamber using a $4 \%$ isoflurane and $\mathrm{O}_{2}$ mixture. During surgery, inhalation anesthesia was delivered through a mask, and the isoflurane flow was adjusted to $2 \%$. Breathing was unassisted in those rats, and blood pressure and gases were not monitored.

Rats were placed on a heating pad in a supine position and an incision was made in the ventral midline of the neck. The common carotid artery was isolated, and then the occipital, superior, and thyroid branches of the external carotid artery (ECA) were isolated and electrocoagulated. A 6-0 silk suture was tied around the distal ECA to 
manipulate the position of the ECA and prevent bleeding. A microvascular clip was also temporarily placed at the origin of the ECA for additional security against bleeding. Distal to the 6-0 suture, the ECA was cut with bipolar electrocoagulation. Another silk suture was loosely tied around the ECA and positioned between the microvascular clip and the 6-0 silk suture. A hole was cut into the ECA stump between the loose and tight silk sutures. A $26 \mathrm{~mm}$ long, 3-0 monofilament nylon suture, with a tip tapered on finegrade sandpaper, was inserted through the hole and past the loose silk suture around the ECA. The loose silk suture was tied to secure the monofilament suture inside the lumen of the ECA. The vascular microclip was then removed, and the monofilament suture was advanced further through the ECA and then through the internal carotid artery, until a faint resistance was encountered followed by a sharp drop in blood pressure. This indicated that the tip of the monofilament suture was sufficiently occluding the origin of the MCA. During ischemia, halothane was used to regulate blood pressure between 65 and $70 \mathrm{mmHg}$. Cases without blood pressure monitoring were maintained on $2 \%$ isoflurane. In either case, the monofilament suture was withdrawn after 60-70 min. To prevent bleeding from the hole that was cut into the ECA, the silk suture that was used to hold the monofilament suture in place was permanently ligated and the ECA stump was electrocoagulated. All wounds were sutured, and gas anesthesia was discontinued to restore blood pressure. Sham surgery $(n=6)$ involved all of the above procedures except the monofilament suture was not advanced into the internal carotid artery (Gharbawie et al., 2008).

\section{Histology}


Rats were deeply anesthetized with sodium pentobarbital and intracardially perfused with $0.9 \%$ phosphate-buffered saline (PBS) then fixed with $4 \%$ paraformaldehyde (PFA) in PBS. The brains were removed and post-fixed in 4\% PFA for $24 \mathrm{~h}$ at $4{ }^{\circ} \mathrm{C}$. They were then placed in cryoprotectant (30\% glucose in $4 \%$ PFA) and sectioned on a microtome after sinking. Serial $40 \mu \mathrm{m}$ sections were obtained from the olfactory bulb to the cerebellum, mounted on gel-coated slides, stained with cresyl violet, and cover slipped.

\section{Cortical Thickness}

The method for measuring cortical thickness was adapted from previously published methods (Kolb and Whishaw, 1981; Kolb and Cioe, 2001). To determine the spatial location of morphological change along the rostrocaudal axis, tissue sections located at $1 \mathrm{~mm}$ intervals were examined, the most rostral section ever measured being located at $+4.7 \mathrm{~mm}$ anterior to bregma, and the most caudal section ever measured being located at $-6.3 \mathrm{~mm}$ posterior to bregma.

For each tissue section, Image $\mathrm{J}$ software (v. 1.38X) was used to take up to twenty measurements of cortical thickness from each coronal section, ten from each hemisphere. For tissue sections 1 and 2, measurements were made along a vector from the center of the hemisphere, out to the edge of the cortex. For all other tissue sections, measurements were made along a vector from the tangent of the outer edge of the corpus callosum to the edge of the cortex. For animals with stroke to the forelimb region of motor cortex 
measurement 1 was taken medial to the infarct, measurement 2 was taken lateral to the infarct, and all successive measurements were taken lateral to the previous measurement. In this manner, the infarct was always located between measurements 1 and 2, and only intact tissue was ever measured in the stroke hemisphere. Two additional measurements were taken in the forelimb area of motor cortex in the five most rostral sections of the intact and control hemispheres. However, only measures outside of the forelimb area of motor cortex were compared across all three groups. For animals with MCAO stroke, all measures were taken medial to the stroke infarct. Analysis of cortical thickness along the coronal axis was performed by grouping all rostrocaudal sections together and making statistical comparisons across all mediolateral measures; likewise, analysis of cortical thickness along the sagittal axis was performed by grouping all mediolateral measures within each coronal section together and making statistical comparisons across all rostrocaudal sections.

Ten and seven tissue sections were measured in pial strip and photothrombotic animals, respectively. Twelve histological tissue sections were measured for animals in the MCAO-C and MCAO-S groups. This was done to ensure that all residual cortical tissue surrounding the infarct was examined, as MCAO infarcts are located more posterior than pial strip or photothrombotic forelimb motor cortex stroke infarcts. For MCAO-C animals, only the 6 most medial measures were analyzed as the last 4 measures often intruded into the infarct in the stroke hemisphere.

\section{Statistical Analysis}


Results were subject to analysis of variance (ANOVA) and follow-up LSD tests with the computer program SPSS (v.16.0). A p-value of less than 0.05 was considered significant. HEMISPHERE (control vs. intact vs. stroke) served as a between-subjects factor. For all ANOVAs, ROSTROCAUDAL SECTION $(1,2,3,4,5,6,7,8,9,10,11$, and 12, depending on stroke model) and MEDIOLATERAL MEASURE (1, 2, 3, 4, 5, 6, $7,8,9$, and 10 depending on stroke model) served as within-subject factors.

\section{Results}

\section{Comparison of cortical thickness in different stroke models}

Figure 10 (top) illustrates representative cresyl violet sections through the center of the infarct from the pial strip (A), small pial strip (B), and photothrombotic occlusion (C) stroke groups and a representative control section (D) at the same rostrocaudal plane. Representative sections of the cortical (E) and subcortical (F) MCAO strokes are shown in relation to a control section $(\mathrm{G})$ in the bottom portion of Figure 10.

Figure 10A shows the damage produced by a pial strip stroke as contrasted with a control section (Fig. 10D). As has been reported in previous studies (Whishaw, 2000;

Gharbawie et al., 2007), the stroke includes a cavity encompassing most of the rostral and caudal regions of the forelimb regions of motor cortex. Although there is some damage to the underlying corpus callosum, due to degeneration of efferent fibers from the motor cortex, other subcortical regions of the brain are not damaged. Figure 10B shows a small pial strip stroke as contrasted with a control section (Fig. 10D). The small pial strip 
$\begin{array}{llll}\text { A. Pial } & \text { B. small Pial } & \text { C. Photo D. Control }\end{array}$
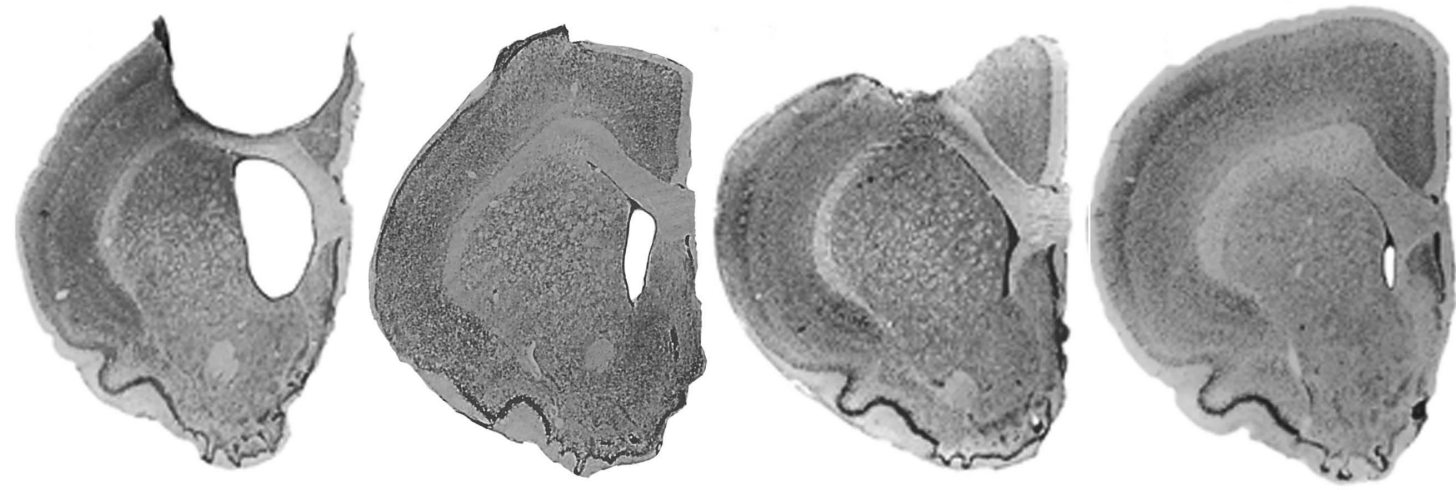

E. MCAO-C

F. MCAO-S

G. Control
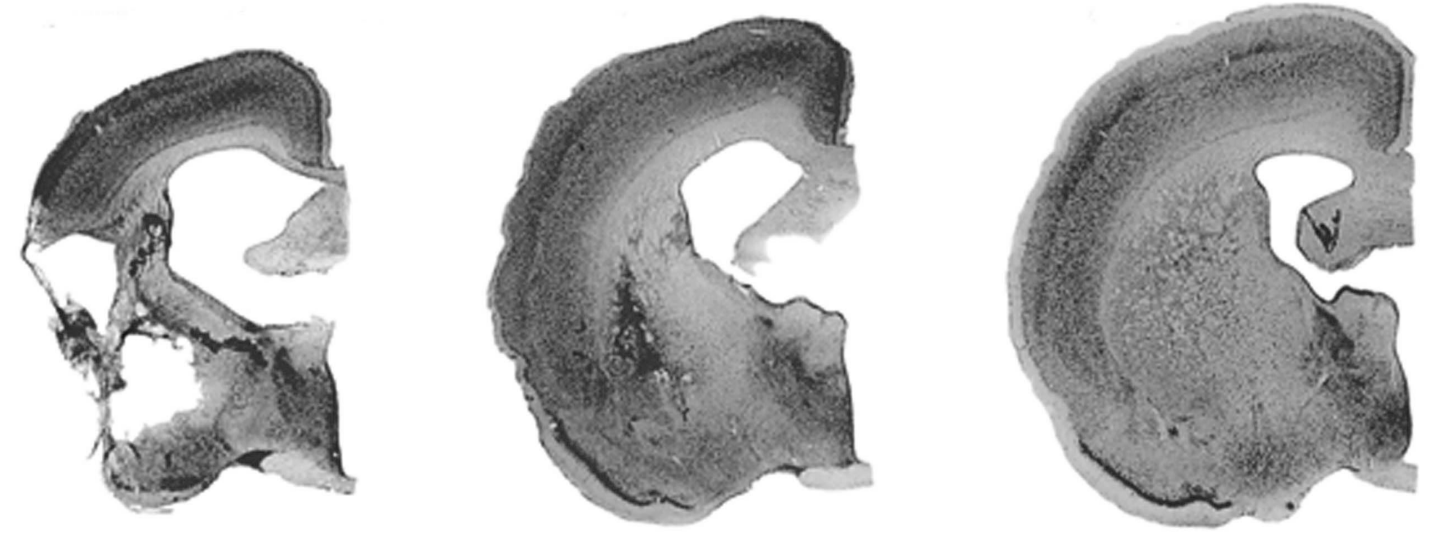

$2 \mathrm{~mm}$ 
Fig. 10. Representative coronal sections (cresyl violet) of the four stroke models and representative control sections collected 31D post-stroke. A. Motor cortex pial strip devascularization. B. Small motor cortex pial strip devascularization. C. Photothrombotic occlusion. D. A control section located at a comparable rostrocaudal location as the center of the pial and photothrombotic infarcts. E. Middle cerebral artery occlusion that includes cortical damage (MCAO-C). F. Middle cerebral artery occlusion with mainly subcortical damage (MCAO-S). G. A control section located at a comparable rostrocaudal location to the center of the middle cerebral artery infarcts. Note: the stroke infarct was localized to the forelimb region of motor cortex in the pial strip devascularization and photothrombotic occlusion models. Cortical damage also occurred in the MCAO-C animals, but not in the MCAO-S animals. (Pial, pial strip stroke; Photo, photothrombotic, MCAO-C, middle cerebral artery, cortical; MCAO-S, middle cerebral artery subcortical). 
infarct is located within motor cortex but does not extend as far medially as the standard pial strip infarct. Also, the underlying corpus callosum and subcortical structures appear are undamaged. Figure 10C shows a photothbrombotic stroke as contrasted with a control section (Fig. 10D). Again, the photothrombotic stroke cavity includes most of the forelimb region of motor cortex. Although there is some damage to the underlying corpus callosum due to degeneration of efferent fibers from the motor cortex, other subcortical regions of the brain are not damaged. The size and location of the pial strip and photothrombotic motor cortex strokes were intentionally equivalent as has been described previously (Shanina et al., 2006; Alaverdashvili et al., 2008). MCAO strokes were variable in size and location with some stokes affecting the lateral neocortex in addition to the caudate-putamen (Fig. 10E) and with other strokes producing damage mainly subcortically to the caudate-putamen (Fig. 10F). Figure 10G illustrates a control tissue section from a rostrocaudal location comparable to the MCAO strokes. The size and location of both MCAO strokes were comparable to previously published reports (Memezawa et al., 1992; Gharbawie et al., 2008). In subsequent descriptions the difference in cortical infarcts were used to divide the MCAO animals into groups that had cortical (MCAO-C) vs. little cortical (MCAO-S) involvement.

\section{General Changes in Residual Cortical Thickness in Multiple Rodent Stroke Models}

Cortical thickness was reduced in both the intact and stroke hemispheres compared to the control group in the small pial strip (Fig. 11A), standard pial strip (Fig. 11B), photothrombotic (Fig. 11C), and MCAO-C (Fig. 11D) stroke models. In all of 

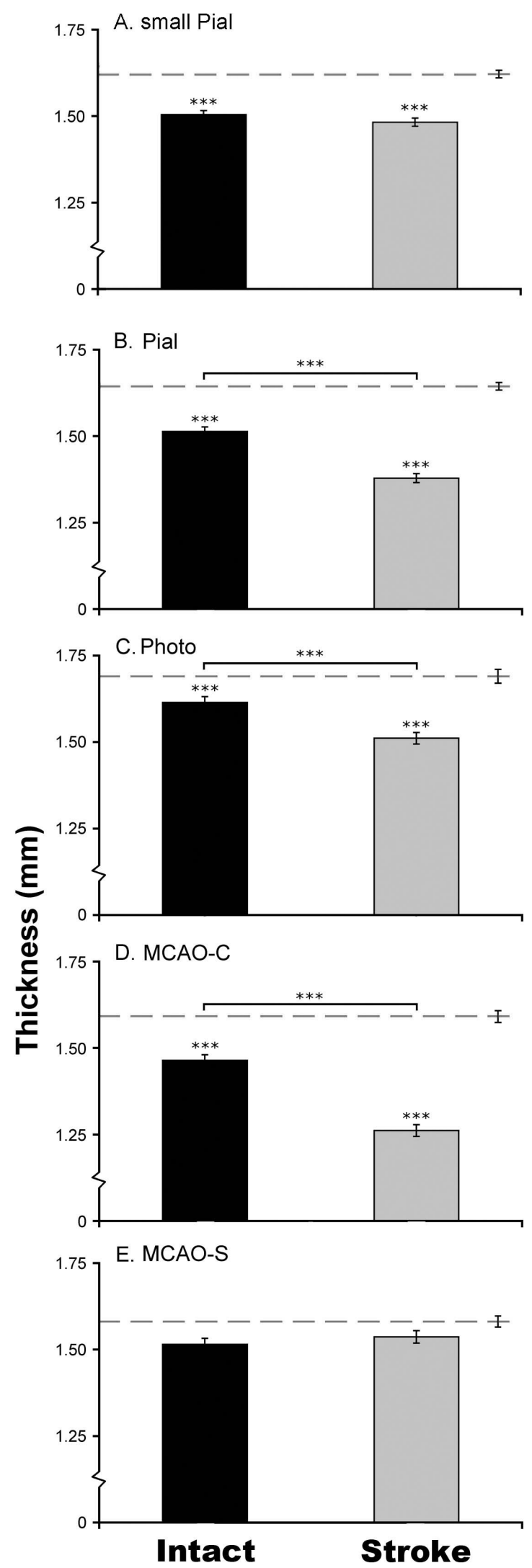
Fig. 11. Cortical thickness in the intact (black) and stroke (gray) hemispheres (mean and standard error) summed over sagittal and coronal measures of the 4 different stroke models at 31D. Note: residual cortical thickness decreased in all animals sustaining focal cortical stroke, irrespective of stroke induction method, size, and location. Damage localized only to subcortical regions did not result in thinning of the overlying cortex. (Dotted line $=$ control groups; refer to Table 1 for subject numbers included in each group; pial - pial strip stroke; photo - photothrombotic stroke; MCAO-C - middle cerebral artery occlusion cortical; $\mathrm{MCAO}-\mathrm{S}$ - middle cerebral artery occlusion subcortical; D - Day; ***p<0.001). 
these models the stroke hemisphere was also significantly thinner than the intact hemisphere, with the exception of the small pial strip stroke group, for which the extent of residual cortical thinning was similar in both the stroke and intact hemispheres.

Figure 11E shows cortical thickness in a MCAO-S stroke model, which was the only stroke model investigated that did not directly damage the cortex. Cortical thickness in the intact and stroke hemispheres of the MCAO-S group did not significantly differ from the control group.

In summary, these results suggest that stroke damage localized to subcortical structures is not sufficient to produce thinning of overlaying cortical tissue. However, cortical thinning appears to be a global consequence of focal cortical stroke, irrespective of the method of stroke induction, size, or location.

These main findings were confirmed by ANOVA's that gave significant effects of HEMISPHERE $[\mathrm{F}(2,25)=11.613, \mathrm{p}<0.001]$ for the small pial strip model, HEMISPHERE $[\mathrm{F}(1,27)=16.139, \mathrm{P}<0.001]$ for the pial strip model, HEMISPHERE $[F(2,13)=93.111, p<0.001]$ for the photothrombotic model and HEMISPHERE $[F(2,17)$ $=27.605, \mathrm{p}<0.001]$ for the MCAO-C model. No significant effect of HEMISPHERE $[F(2,12)=2.768, \mathrm{p}>0.05]$ was found for the MCAO-S model. Follow-up post hoc analyses confirmed that cortex in the stroke hemisphere was significantly thinner compared to the intact hemisphere in the pial strip, photothrombotic, and MCAO-C models $(\mathrm{p}<0.001)$.

\section{Small Pial Stip Devascularization of Forelimb Motor Cortex}


Figure 12 summarizes the extent of cortical thinning that occurs 31 days poststroke in a motor cortex pial strip devascularization model, with a smaller stroke infarct (small Pial). In this model cortical thickness decreased in both the intact and stroke hemispheres 31 days post-stroke. However, unlike the larger motor cortex pial strip devascularization model, the extent of cortical thinning was comparable between the intact and stroke hemispheres. Along the coronal (mediolateral) axis, the greatest reduction in cortical thickness occurred proximal to the infarct but extended to distal lateral tissue in both the intact and stroke hemispheres (Fig. 12A). Along the sagittal (rostrocaudal) axis, cortical thickness decreased in tissue proximal, and immediately caudal, to the infarct (Fig. 12B). This effect however, was not statistically significant. These results were confirmed by ANOVAs that gave significant effects of HEMISPHERE $[\mathrm{F}(2,25)=11.613, \mathrm{p}<0.001]$ and HEMISPHERE X MEASURE $[\mathrm{F}(18,225)=2.094, \mathrm{p}<0.01]$, but not HEMISPHERE X SECTION $[\mathrm{F}(18,225)=1.102$, $\mathrm{p}>0.05]$.

\section{Photothrombotic Occlusion of Forelimb Motor Cortex}

Figure 13 summarizes the extent of cortical thinning that occurs 31 days poststroke in a motor cortex photothrombotic occlusion stroke model. In this model cortical thickness decreased in both the intact and stroke hemispheres by 31 days post-stroke. Significant cortical thinning occurred along the coronal (mediolateral) axis. Along this axis, the greatest reductions in cortical thickness occurred in the stroke hemisphere, immediately lateral to the stroke infarct but also extended to distal cortical tissue as well 


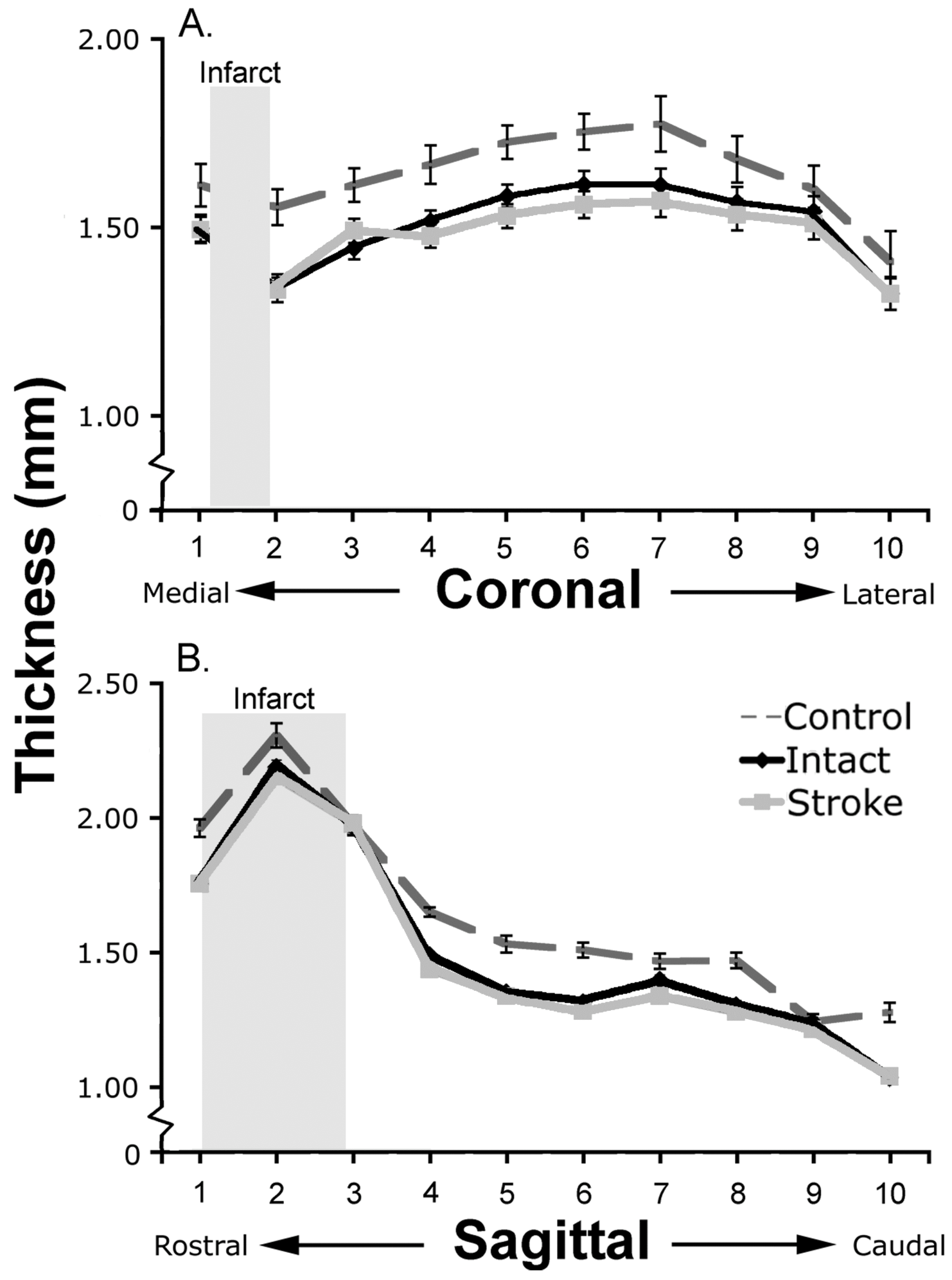


Fig. 12. Cortical thickness (mean and standard error) of coronal (A) and sagittal (B) points of measurement from non-stroke control rats (dotted line, $n=4$ ), the intact hemisphere of sMC stroke rats (black, $n=12$ ), and the stroke hemisphere of sMC stroke rats (gray, $n=12$ ) 31 days post-stroke. Note: Cortical thickness decreased in both the stroke and intact hemispheres in tissue lateral to the stroke infarct. Thinning was greatest proximal to the infarct but extended to distal tissue as well. ( $\mathrm{sMC}=$ small Motor Cortex pial strip). 


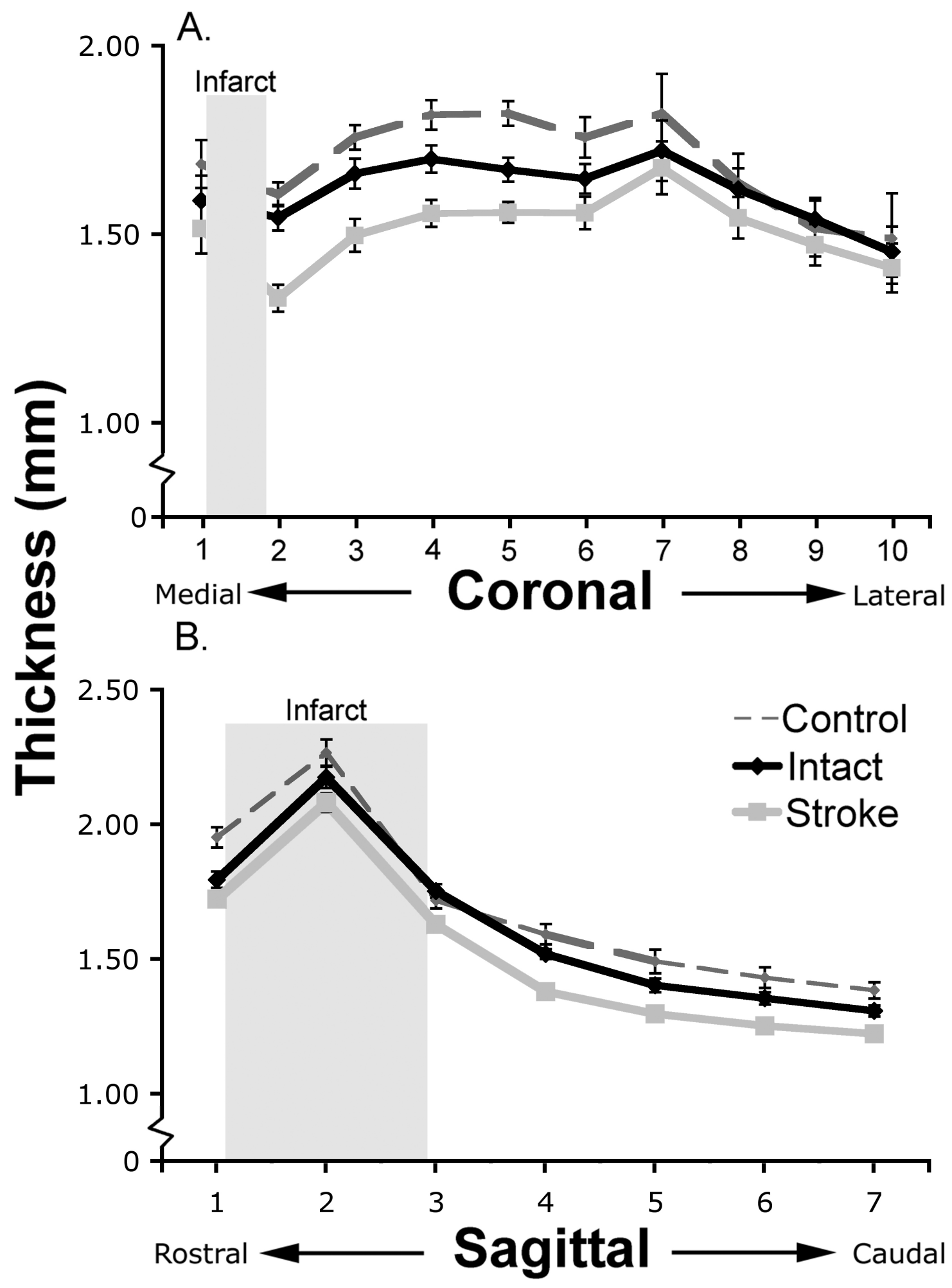


Fig. 13. Cortical thickness (mean and standard error) of coronal (A) and sagittal (B) points of measurement from non-stroke control rats (dotted line, $n=4$ ), the intact hemisphere of photothrombotic stroke rats (black, $n=6$ ), and the stroke hemisphere of photothrombotic stroke rats (gray, $\mathrm{n}=6$ ) 31 days post-stroke. Note: Reductions in cortical thickness were greatest immediately lateral to the stroke infarct but extended laterally into considerably distal cortical tissue as well. (Shading represents histological planes containing the infarct). 
(Fig. 13A). No significant decrease in cortical thickness occurred along the sagittal (rostrocaudal) axis (Fig. 13B).

These results were confirmed by ANOVAs that gave significant effects of HEMISPHERE $[\mathrm{F}(2,13)=93.111, \mathrm{p}<0.001]$ and HEMISPHERE X MEASURE $[\mathrm{F}(18,117)=7.469, \mathrm{p}<0.001]$, but not HEMISPHERE X SECTION $[\mathrm{F}(12,78)=0.839$, $\mathrm{p}>0.05]$.

\section{Middle Cerebral Artery Occlusion of Subcortical and Cortical Areas (MCAO-C)}

Figure 14 summarizes the extent of cortical thinning that occurs 31 days poststroke in a MCAO-C stroke model. Cortical thickness decreased significantly, in both the intact and stroke hemispheres. Cortical thinning extended a large distance from the stroke infarct along the coronal (mediolateral axis). Extensive thinning occurred immediately medial to the infarct and continued to extend into distal medial tissue in both the intact and stroke hemispheres (Fig. 14A). Along the sagittal (rostrocaudal) axis, cortical thinning occurred in tissue surrounding the infarct and continued into distal anterior tissue, largely in the stroke hemisphere, but also in a lesser extent in the intact hemisphere (Fig. 14B).

These results were confirmed by ANOVAs that gave significant effects of HEMISPHERE $[\mathrm{F}(2,17)=27.605, \mathrm{p}<0.001]$, HEMISPHERE X SECTION $[\mathrm{F}(22,187)=$ 2.056, $\mathrm{p}<0.01]$. and HEMISPHERE X MEASURE $[\mathrm{F}(10,85)=6.486, \mathrm{p}<0.001]$. 


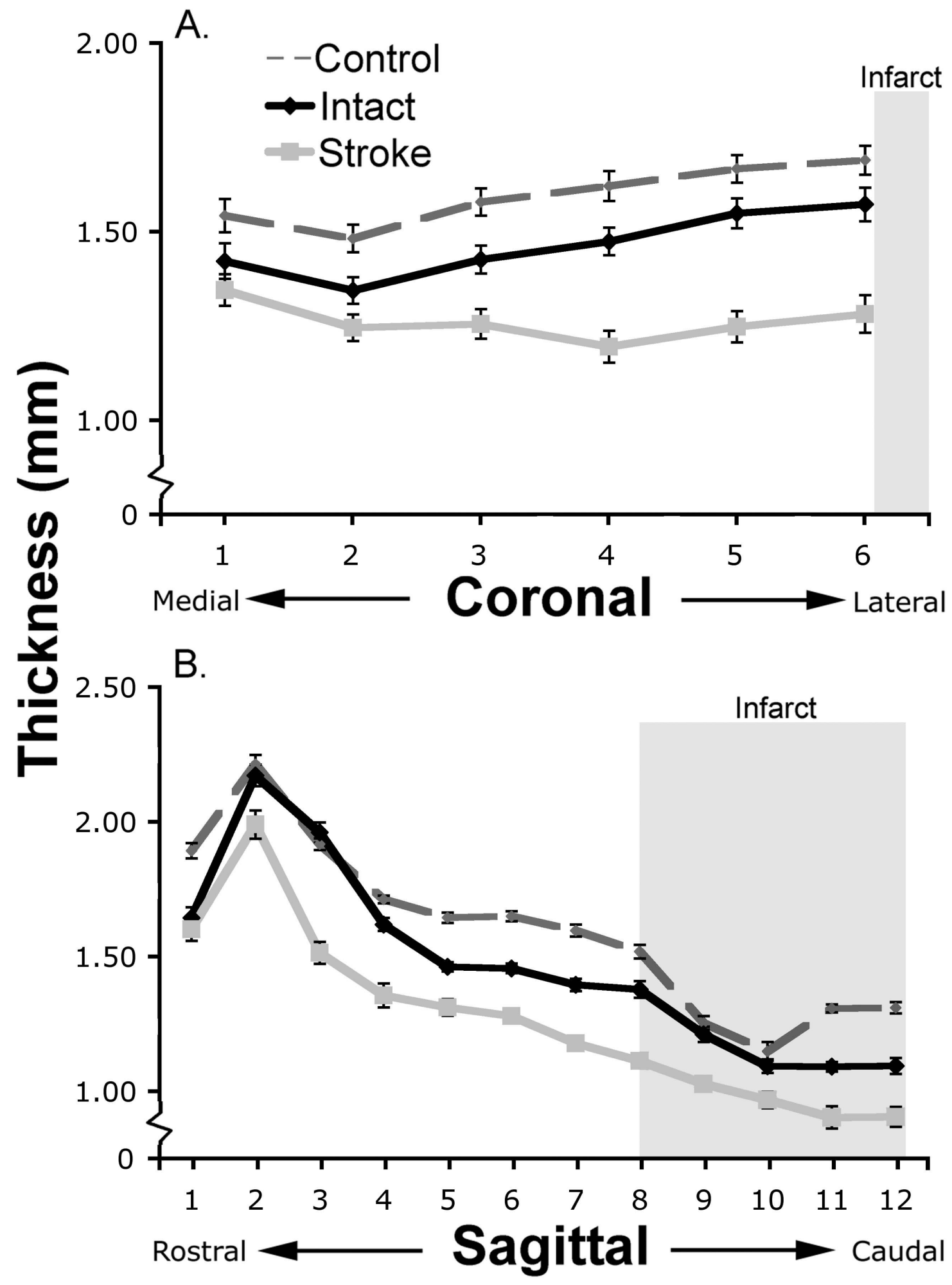


Fig. 14. Cortical thickness (mean and standard error) of coronal (A) and sagittal (B) points of measurement from non-stroke control rats (dotted line, $n=6$ ), the intact hemisphere of MCAO-C stroke rats (black, $\mathrm{n}=7$ ), and the stroke hemisphere of MCAOC stroke rats (gray, $n=7$ ) 31 days post-stroke. Note: Reductions in cortical thickness were greatest in the stroke hemisphere and proximal to the infarct but extended into distal cortical tissue and the intact hemisphere as well. (MCAO-C - Middle cerebral artery occlusion with cortical and subcortical damage; shading represents histological planes containing the infarct). 


\section{Middle Cerebral Artery Occlusion of Subcortical Areas (MCAO-S)}

Figure 15 summarizes the change in cortical thickness 31 days post-stroke in an MCAO-S stroke model. Cortical thickness did not significantly decrease along the coronal (mediolateral) axis (Fig. 15A), or sagittal (rostrocaudal) axis (Fig. 15B) in either the intact or stroke hemispheres of the MCAO-S model, which spares overlaying cortex.

These results were confirmed by ANOVAs that gave significant effects of $\operatorname{HEMISPHERE}[\mathrm{F}(2,13)=2.768, \mathrm{p}>0.05]$.

\section{Discussion}

No single rodent stroke model fully mimics the human stroke condition, thus the present study included an investigation of a number of stroke models, including two models of forelimb motor cortex stroke, pial stripping and photothrombotic stroke, that have been widely used to investigate functional recovery of forelimb movement following stroke (Alaverdashvili et al., 2008; Kleim et al., 2007; Whishaw et al., 1991; Shanina 2006). Because occlusion of the MCA composes a large proportion of clinical stroke cases, the study also included a model of MCAO stroke that produces varying degrees of damage. One group of MCAO rats sustained mainly basal ganglia damage and another group had additional sensorimotor cortex damage. It was expected that this comparison would indicate whether the anatomical changes in cortical thickness were linked to forelimb motor cortex damage specifically, to cortical damage more generally, or to any type of brain damage. The results indicate that thinning of residual cortical 


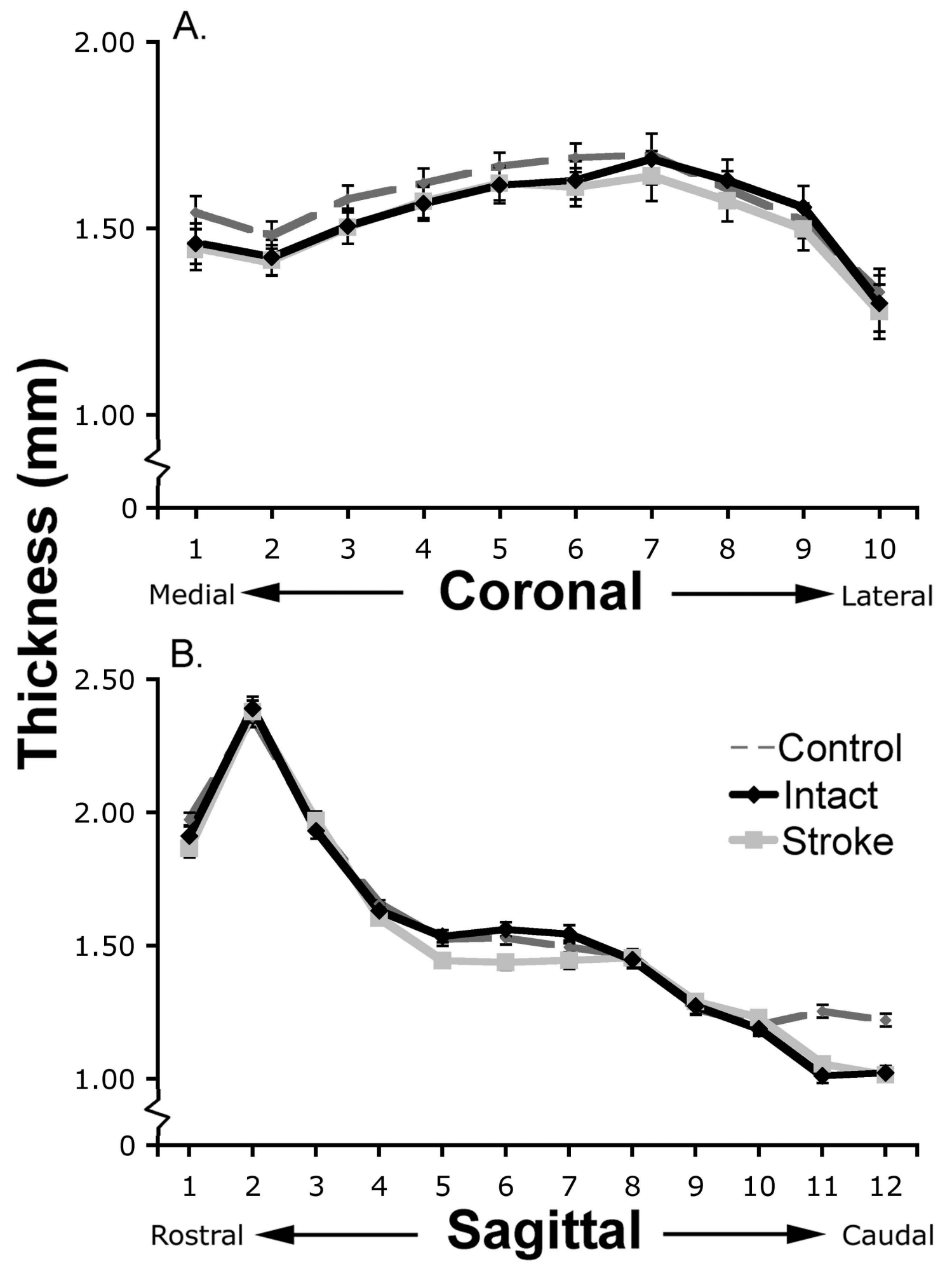


Fig. 15. Cortical thickness (mean and standard error) of coronal (A) and sagittal (B) points of measurement from non-stroke control rats (dotted line, $n=6$ ), the intact hemisphere of MCAO-S stroke rats (black, $n=5$ ), and the stroke hemisphere of MCAO$\mathrm{S}$ stroke rats (gray, $\mathrm{n}=5$ ) 31 days post-stroke. Note: Cortical thickness did not decrease in a stroke model that did not induce a cortical infarct. (MCAO-S - Middle cerebral artery occlusion with only subcortical damage; shading represents histological planes containing the infarct). 
tissue is a general consequence of focal cortical stroke, irrespective of stroke induction method, size, or location. Stroke localized to subcortical structures however, did not induce thinning of the overlying cortex.

The mechanism of stroke induction varied between the different stroke models used in the present study, however, the extent of subsequent cortical thinning was similar provided that the cortex was damaged. A number of mechanisms likely contributed to the similarity. First, it has been suggested that cortex can thin by moving into the infarct cavity. This would be especially notable in the pial strip model in which tissue can move into not only the infarct, but also into the cavity produced by the trephination itself (Adams et al., 1994; Kolb \& Holmes, 1983; Navari et al., 1978; Whishaw, 2000; Xu et al., 2007). Second, all of the stroke models likely involve neuroinflammatory responses, secondary neurodegeneration, and apoptosis, which take place over time following the stroke (Chen et al., 2003; Bidmon et al., 1998; Pulsinelli, 1982; Witte et al., 2000). Third, there are likely widespread changes involving the loss of neuropile and adjustments in surviving neurons that contribute to time-dependent cortical alterations (Butefisch, 2006; Jones, 1999; Jones et al., 1996, 1999). These processes might also contribute to changes in the contralateral cortex although this hemisphere underwent no direct damage from the stroke. It is interesting that cortical thinning did not follow damage that was restricted to the basal ganglia. Other work however, suggests that such damage, even though it includes damage to corticofugal fibers, does not result in cell death in the neocortex (Tetzlaff et al., 1994; Whishaw et al., 1993). Nonetheless, infiltration of astrocytes and 
microglia is known to occur after stroke. Thus, it is possible that a certain amount of cortical thickness may be maintained by the presence of these cells, and the extent of cell death in the cortex may be underestimated by cortical thickness measures alone.

For the focal cortical stroke models, the general spatial pattern of thinning was similar, with the greatest losses in cortical thickness occurring proximal to the stroke infarct, but extending to distal cortical tissue and also the intact hemisphere. This is noteworthy because the pial strip and photothrombotic models do not undergo significant reperfusion and thus produce only a very small penumbra zone compared to the MCAO model. Thus, it appears that early degeneration within proximal penumbral tissue is not necessarily an important contributor to thinning of residual cortical tissue.

The formation of a cortical infarct develops gradually over 4 to 5 days post-stroke (Garcia et al., 1995). Thus, it is interesting that previous work has shown that the majority of cortical thinning occurs in the chronic post-stroke time period, after formation of the infarct (Karl et al., 2010). Because the results of the present study suggest that a physical cortical infarct is necessary for subsequent thinning of residual tissue, it is plausible that if formation of the infarct were to be instant, thinning of residual cortical tissue could begin in the acute post-stroke period. The present study is limited in that it did not include an aspiration stroke model, which could have addressed this question. In an aspiration model, the stroke is induced by suctioning out the target tissue, resulting in the instant formation of a cortical infarct (Gonzalez and Kolb, 2003). Interestingly, Kolb (1987) has observed thinning and collapse of residual cortical tissue into the infarct immediately after aspiration stroke in neonatal rats. These findings suggest that the space 
created by a cortical infarct may be more important than post-stroke time when considering the extent of residual tissue thinning.

One mechanism by which "space" created by the infarct may lead to thinning of proximal and distal peri-infarct tissue is by diminishing gross structural support for surrounding tissue, which may then stretch or fall into the open space. The idea that the size of a cortical infarct may influence the extent of peri-infarct tissue thinning is supported by the present results, which show that small strokes in the motor cortex produce the least amount of subsequent thinning in the stroke hemisphere, compared to MCAO-C strokes, which produced the largest cortical infarct and the largest amount of post-stroke thinning.

In conclusion, thinning of peri-infarct tissue appears to be a general consequence of focal cortical stroke, irrespective of stroke method or location. The amount of cortical thinning, however, may be related to the size of the infarct. Thinning is greatest in proximal peri-infarct tissue in the stroke hemisphere, but extends to distal and contralateral tissue as well. Thus, preclinical investigations of peri-infarct tissue in focal cortical stroke models must consider gross morphological changes such as cortical thinning when describing the function of peri-infarct tissue. 


\title{
CHAPTER FOUR
}

\section{SPATIOTEMPORAL CHANGES IN MRI CORTICAL THICKNESS AFTER PIAL STRIP DEVASCULARIZATION SROKE OF THE FORELIMB AREA OF MOTOR CORTEX IN THE ADULT RAT: THE CONTRIBUTION OF TISSUE MOVEMENT}

\begin{abstract}
Morphological changes in peri-infarct tissue, such as tissue thickness, volume, and density loss have been observed after stroke in the adult rat. These changes are dependent on the formation of a cortical infarct as cortical thinning is not observed when the stroke infarct is localized to subcortical structures. It is possible that movement of peri-infarct tissue into the stroke infarct contributes to morphological change in both proximal and distal residual cortical tissue. The purpose of the present study was to document the extent of peri-infarct tissue movement and thinning using magnetic resonance imaging (MRI) after focal stroke of motor cortex in the adult rat. MRI provides in vivo observation of morphological change and avoids the morphological anomalies known to occur in histological tissue, such as artificial tissue shrinkage and deformation. LongEvans hooded rats received unilateral pial strip devascularization stroke to the forelimb area of motor cortex as well as insertion of markers of cortical tissue location. Animals then underwent serial MR imaging at 1 hour, 1, 3, 7, 14, and 31 days post-stroke. Decreases in cortical thickness were similar to previous findings in post-stroke histological tissue and were found to extend far beyond the stroke infarct and included
\end{abstract}


most of the sensorimotor regions of the stroke and intact hemispheres. Peri-infarct tissue, even distal to the stroke infarct, was found to move towards the stroke infarct. The results are discussed in relation to the idea that movement of "intact" residual tissue into a cortical infarct could be related to the molecular, electrophysiological, and morphological changes previously observed in distal post-stroke cortex.

\section{Introduction}

Dynamic changes observed in peri-infarct tissue have been suggested to mediate post-stroke functional recovery (Fridman et al., 2004; Frost et al., 2003; Gharbawie et al. 2007; Kolb et al., 2001; Liu and Rouiller, 1999; Nudo et al., 1996; Schallert et al., 2000; Whishaw, 2000). Peri-infarct changes include thinning, volume loss, and density loss in histological tissue (Karl et al., 2010), as well as time-dependent normalization of T2 and fractional diffusion anisotropy (van der Zijden et al., 2008b) as identified by magnetic resonance imaging (MRI). It has been proposed that these changes may be indicative of edema resolution (Lin et al., 2002a,b), glial proliferation (Ishii et al., 1998; Wegener et al., 1996), and possibly white matter reorganization or restoration of neuronal connectivity (Jiang et al., 2006; van der Zijden et al., 2008a). In addition, the formation of a cortical infarct has been shown to be necessary for the development of subsequent cortical thinning, as stroke localized to the basal ganglia does not lead to thinning of the overlying cortex (Karl et al., 2010). It is possible that the formation of a cortical infarct facilitates additional morphological changes in peri-infarct tissue by destabilizing proximal peri-infarct tissue, which may then "fall" into the stroke infarct. "Filling in" of 
the stroke core by peri-infarct tissue has been observed after neonatal stroke (Kolb and Whishaw, 1985, Whishaw, 2000) and it is likely that any proximal movement of tissue will have morphological consequences for distal tissue as well. The nature and extent of post-stroke tissue movement however, has not been fully documented in the adult rat.

The purpose of the present study was to use MRI to document the extent of periinfarct tissue movement after focal cortical stroke in the adult rat and to determine if such stretching is related to the thinning of both proximal and distal cortical tissue that occurs after stroke in the adult rat. Post-stroke morphological change in the rat has been almost exclusively studied in post mortem, histological tissue. This presents a problem however, as the histological preparation of neural tissue is known to introduce a number of morphological anomalies. Tissue perfusion, removal, staining, and dehydration can lead to artificial tissue shrinkage, nicks in surface brain areas, and changes in tissue shape and location (Garman, 1990; Overgaard and Meden, 2000; Sadowski et al., 1995; Simmons and Swanson, 2009; Skoglund et al., 1996). It is possible that any cortical thinning, movement, volume, or density loss observed after stroke in the adult rat could be exaggerated by histological preparation. MRI however, allows for longitudinal investigation of morphological change in the same animals over long periods of time and avoids the morphological confounds of histological tissue preparation.

The effect of forelimb motor cortex stroke on residual cortical thickness was examined in vivo in the adult rat. Animals received standard motor cortex pial strip devascularization stroke $(n=4)$ as well as six temporary cannula insertions into surrounding cortical tissue, 3 per hemisphere. Upon removal of the cannula, a residual vertical tract is created in the cortical lamina. The location and orientation of these tracts 
can be seen in MRI and histological tissue and can act as markers of tissue location. Rats underwent serial MR imaging at the following time points: Pre-surgery, 1 hour $(\mathrm{H}), 1$ day (D), 3D, 7D, 14D, and 31D. Animals were sacrificed immediately following the final imaging session and all brains were collected and processed for cresyl violet staining. Measures of cortical thickness were obtained from MR images and the location of the cortical cannula tracts was evaluated in histological and MR images to determine the extent of post-stroke tissue movement.

\section{Methods and Materials}

\section{Subjects}

Subjects were male Long-Evans hooded rats, 90 days of age, and weighing 350550g. Rats were housed in groups of two in Plexiglas cages with cob bedding. The colony room in which they were housed was temperature and humidity controlled and maintained on a $12 \mathrm{~h} / 12 \mathrm{~h}$ light/dark cycle. Purina rat chow and water were available ad libidum. All experiments complied with the guidelines of the University of Lethbridge Animal Care Committee as set out by the Canadian Council for Animal Care.

\section{Standard Pial Strip Devascularization Stroke (MC) with Anatomical Markers of Tissue Location}


The forelimb region of the motor cortex, as previously defined by behavioral (Whishaw 2000), anatomical (Donoghue and Wise, 1982), and electrophysiological (Hall and Lindhom, 1974; Donoghue and Wise 1982) studies was targeted for devascularization. Animals received an injection of atropine methyl nitrate $(0.1 \mathrm{mg} / \mathrm{kg}$ i.p.; Sigma-Aldrich, St. Louis, MO) to facilitate respiration throughout surgery, burprenorphine ( $0.05 \mathrm{mg} / \mathrm{kg}$ s.c.; Schering-Plough, Hertfordshire, UK) as an analgesic, and an injection of sodium pentobarbital (65 mg/kg, i.p.; Sigma-Aldrich, St. Louis, MO) for anaesthetization. A small incision was made in the scalp along the midsagittal suture and a fine dental burr was used to drill four holes in the skull above motor cortex. The coordinates of these holes were measured from Bregma; i) Anterior (A) -1.0 mm, lateral (L) $+1.0 \mathrm{~mm}$; ii) A -1.0 mm, L +4.0 mm; iii) A +4.0 mm, L +4.0 mm; iv) A +4.0 mm, L $+1.0 \mathrm{~mm}$. The rectangular area of skull demarked by the four holes was then removed using a dental burr and the dura mater below the trephination was cut and peeled away. The exposed motor cortex was devascularized by gently wiping away the pia and removing blood vessels with a saline-soaked cotton swab. This procedure does not involve physical removal of the affected tissue. Thus, the stroke cavity remained filled with tissue until the degenerating debris was cleared by glia.

Rats also received six insertions ( 3 per hemisphere) of a small 28 gauge cannula to a depth of $5 \mathrm{~mm}$ below the surface of the cortex. The cannula was immediately removed. This procedure produces a vertical lesion in the cortex, which is visible in histological tissue as well as in MR images due to a change in tissue perfusion density and other tissue changes including infiltration of astrocytes to the site of the lesion tract. The coordinates of the cannula insertions were: i) A $+3.7 \mathrm{~mm}, \mathrm{~L} \pm 4.2 \mathrm{~mm}$, 
dorsal/ventral (DV) $5.0 \mathrm{~mm}$; ii) A $+1.7 \mathrm{~mm}, \mathrm{~L} \pm 4.2 \mathrm{~mm}$, DV $5 \mathrm{~mm}$; iii) A $-1.6 \mathrm{~mm}, \mathrm{~L} \pm$ $4.2 \mathrm{~mm}$, DV $5 \mathrm{~mm}$.

The motor cortex trephination was left uncovered, the incision sutured closed, and the animal's condition monitored in a recovery room for 24 hours. Sham-operated animals were treated similarly except that the dental burr did not perforate the skull, and the processes of trephination and devascularization were not performed.

\section{Magnetic Resonance Imaging (MRI)}

Rats were anaesthetized by inducing them with $4 \%$, and maintaining them at 1.5 $2.0 \%$, isoflurane gas while in the MRI magnet. The animals were placed in a MRcompatible stereotaxic device (Kopf, CA), and immobilized with earbars and a toothbar. Heart rate and blood oxygen saturation were monitored during MRI using MouseOx software (Starr Lifesciences Corp., Allison Park, Pennsylvania), and body temperature was maintained at $37.0 \pm 1.0^{\circ} \mathrm{C}$. MRI was performed using an Oxford (Oxford, UK) 4.7T horizontal $300 \mathrm{~mm}$ bore MR magnet with a SMIS console (Surry, UK) and a home-built quadrature bird cage volume coil (50 $\mathrm{mm}$ diameter, $54 \mathrm{~mm}$ length) for signal excitation and detection. T2-weighted Fast Spin Echo (FSE) (TR/TE = 3000/90ms; echo train length $=8$; acquisition matrix $=256 \times 256$; voxel resolution $=0.31 \times 0.31 \times 1.5 \mathrm{~mm}^{3}$; slice thickness $=1.5 \mathrm{~mm}$, slice separation $=2 \mathrm{~mm}$; of coronal images acquired $=8$; total imaging time $=3.2 \mathrm{~min})$ and $3 \mathrm{D}$ MRI $\left(\mathrm{TR} / \mathrm{TE}=100 / 6 \mathrm{~ms}\right.$; flip angle $=70^{\circ}$; acquisition matrix $=128 \times 128 \times 32$; voxel resolution $=0.39 \times 0.39 \times 0.47 \mathrm{~mm}^{3}$; slice thickness $=$ 
$470 \mu \mathrm{m}$; $\#$ of coronal images acquired $=32$; total imaging time $=6.8 \mathrm{~min}$ ) data sets were acquired.

\section{Histology}

Rats were deeply anesthetized with sodium pentobarbital and intracardially perfused with $0.9 \%$ phosphate-buffered saline (PBS) then fixed with $4 \%$

paraformaldehyde (PFA) in PBS. The brains were removed and post-fixed in 4\% PFA for $24 \mathrm{~h}$ at $4{ }^{\circ} \mathrm{C}$. They were then placed in cryoprotectant (30\% glucose in $4 \%$ PFA) and sectioned on a microtome after sinking. Serial $40 \mu \mathrm{m}$ sections were obtained from the olfactory bulb to the cerebellum, mounted on gel-coated slides, stained with cresyl violet, and cover slipped.

\section{Cytoarchitectural Tissue Movement Analysis}

Cytoarchitectural analysis was performed on chronic pial strip devascularization histological tissue (7D to 31D) to determine the extent of post-stroke tissue movement. Three coronal sections, encompassing the stroke infarct were analyzed from each rat. Photographs of Nissl-stained tissue were converted to 32-bit grey scale images and were analyzed using Image J software (v. 1.38X). In the stroke and intact hemisphere a black line was drawn from the dorsal edge of the corpus callosum to the outer edge of the cortex such that it intersected the boundary between the darkly stained pyramidal cells in the somatosensory cortex (lateral to the black line) and the lighter stained pyramidal cells 
of the motor cortex (medial to the black line, see figure 11C). The distance between the outer tip of the black line (boundary of the somatosensory and motor cortices), and the midline of the brain was then measured.

\section{Cortical Thickness}

Because the voxel depth of FSE images was $1.50 \mathrm{~mm}$ and gapped at $2 \mathrm{~mm}$, only six FSE images per rat were analyzed at each time point. The center of these six FSE images approximately corresponded (in location) to every other histological section that was analyzed from histological tissue of the pial strip stroke group. Cortical thickness in FSE images was evaluated using the same measuring method as described earlier for the cresyl violet sections. For each tissue section, Image J software (v. 1.38X) was used to take up to twenty measurements of cortical thickness from each coronal section, ten from each hemisphere. For tissue sections 1 and 2, measurements were made along a vector from the center of the hemisphere, out to the edge of the cortex. For all other tissue sections, measurements were made along a vector from the tangent of the outer edge of the corpus callosum to the edge of the cortex. For animals with stroke to the forelimb region of motor cortex measurement 1 was taken medial to the infarct, measurement 2 was taken lateral to the infarct, and all successive measurements were taken lateral to the previous measurement. In this manner, the infarct was always located between measurements 1 and 2, and only intact tissue was ever measured in the stroke hemisphere. Two additional measurements were taken in the forelimb area of motor cortex in the five most rostral sections of the intact and control hemispheres. However, 
only measures outside of the forelimb area of motor cortex were compared across all three groups.

\section{Tissue Marker Visualization}

Reconstruction of cannula tracts in the cortical layers of histological tissue from rats that received cannula insertions was accomplished by projecting cresyl violet stained sections on a Zeiss 2 POL projector set at a magnification of 13X. Major anatomical landmarks from each section were manually traced in black ink, while the location of the cannula tract was traced in red ink. In vivo identification of cannula tracts was possible in 3D MRI slices due to the small slice thickness $(470 \mu \mathrm{m})$ obtained with this data acquisition sequence. This allowed for visualization of changes in tissue perfusion density and made it possible to identify the location at which cannulas had been inserted into intact tissue at the time of stroke induction.

\section{Statistical Analysis}

Results were subject to analysis of variance (ANOVA) and follow-up LSD tests with the computer program SPSS (v.16.0). A p-value of less than 0.05 was considered significant. TIME (1H, 1D, 3D, 7D, 14D, and 31D), HEMISPHERE (control vs. intact vs. stroke), ROSTROCAUDAL SECTION (1, 2, 3, 4, 5, and 6), and MEDIOLATERAL MEASURE $(1,2,3,4,5,6,7,8,9,10)$ served as within-subject factors. 


\section{Results}

\section{Cortical thickness in MRI sections}

Figure 16 shows cortical thickness obtained from repeated imaging sessions $(1 \mathrm{H}$ to $31 \mathrm{D}$ ) on 4 animals, summarized over all mediolateral coronal and all rostrocaudal sagittal MRI measures, after pial strip motor cortex stroke.

Figure 16A shows that the cortex was thinner in the stroke and intact hemispheres compared to pre-surgery cortical thickness, thinner in the chronic period than the acute period, and thinner in the stroke compared to the intact hemisphere. Figure 16B summarizes cortical thickness in the intact vs. stroke hemisphere in the acute and chronic periods. The greatest reduction in cortical thickness occurred in the stroke hemisphere in the chronic time period.

These findings confirm that thinning of residual cortical tissue is not unique to histological tissue, but also develops in living post-stroke tissue. Furthermore, the pattern of cortical thinning development over time is similar in both instances, with the greatest thinning occurring in the stroke hemisphere in the chronic time period. One difference is the immediate $(1 \mathrm{H})$ drop in cortical thickness in vivo. It is possible that this thinning is due to dehydration of the overall brain following pial-strip devascularization stroke surgery compared to the intact, pre-surgery brain. In histological tissue, artificial dehydration occurs in both control and stroke tissue and this difference would not be evident.

These main findings were confirmed by ANOVAs that gave significant effects of 


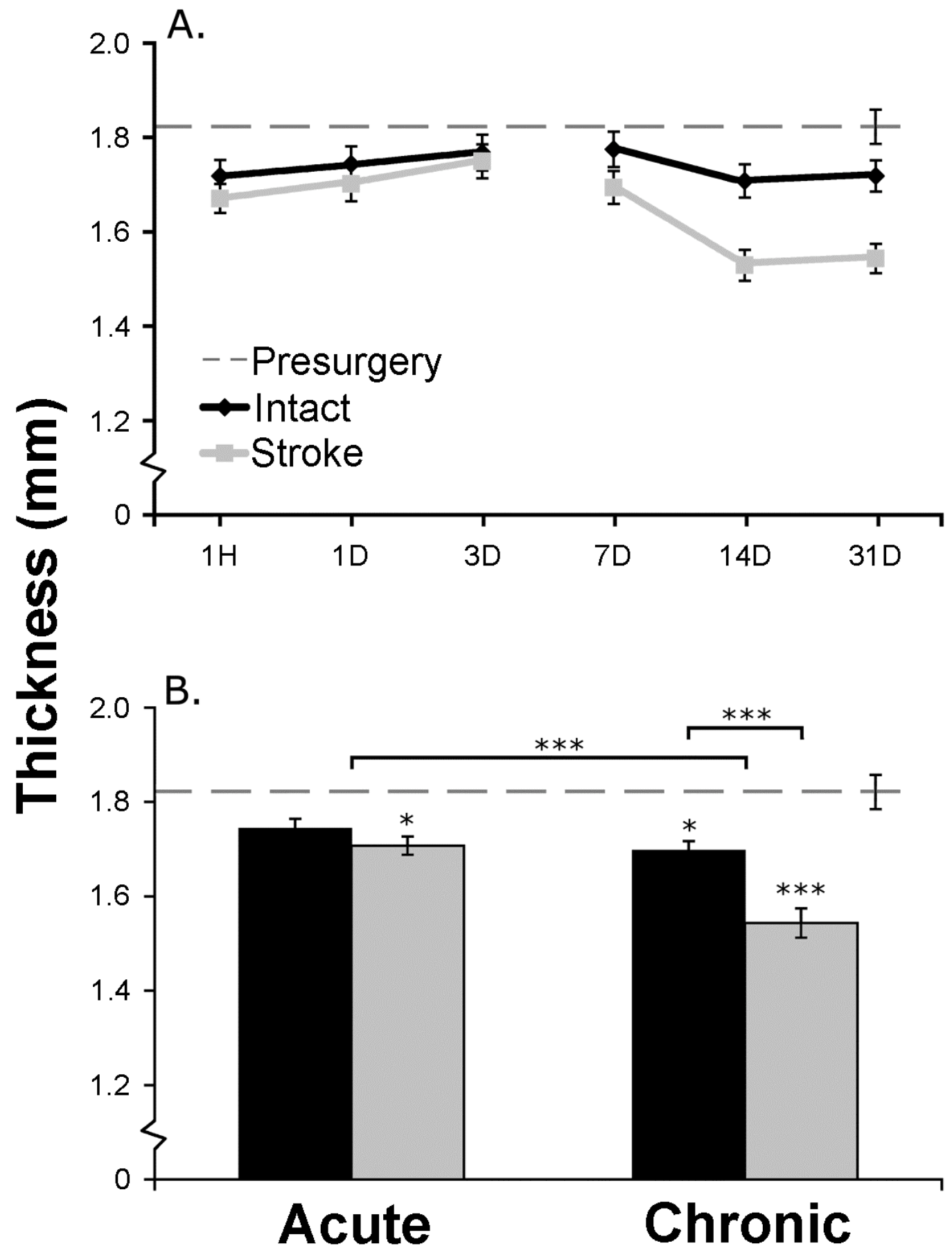


Fig. 16. Cortical thickness (mean and standard error) summed over sagittal and coronal MRI measures at different post-stroke acute $(1 \mathrm{H}-3 \mathrm{D})$ and chronic $(7 \mathrm{D}-31 \mathrm{D})$ times. A. Cortical thickness of MRI animals before stroke surgery (dotted line, $n=4$ ), the intact hemisphere after stroke (black, $n=4$ ), and the stroke hemisphere after stroke (gray, $\mathrm{n}=$ 4) at acute and chronic time points. B. Mean cortical thickness in the acute period vs. chronic period. Note: cortical thinning is progressive in vivo in both hemispheres after stroke with the stroke hemisphere most affected in the chronic period, (MRI, Magnetic Resonance Imaging; H - Hour; D - Day; $\left.{ }^{*} \mathrm{p}<0.05, * * * \mathrm{p}<0.001\right)$. 
HEMISPHERE $[\mathrm{F}(1,3)=147.97, \mathrm{p}<0.001]$, and TIME X HEMISPHERE $[\mathrm{F}(6,18)=$ 5.377, $\mathrm{p}<0.001]$. No significant effect of $\operatorname{TIME}[\mathrm{F}(6,18)=1.575, \mathrm{p}>0.05]$ was found. Follow-up post hoc analyses confirmed that cortical thickness was significantly reduced in the stroke hemisphere compared to the intact hemisphere and to the pre-surgery control measures in the chronic period.

\section{Coronal and Sagittal Cortical Thickness in MR Sections}

Figure 17 gives a summary of cortical thickness in MR images acquired in the chronic period (7D to 31D).

Figure 17A shows measures of cortical thickness from 10 mediolateral coronal locations summarized across 10 rostrocaudal sections. The first measurement location was taken medial to the infarct while the remaining measurement locations were taken lateral to the infarct (gray bar). Decreases in cortical thickness were greatest in the stroke hemisphere but extended to the intact hemisphere as well. Thinning was greatest immediately lateral to the infarct but extended to distal tissue as well.

Figure 17B illustrates the change in residual cortical thickness from just posterior of the olfactory bulb back to the most caudal section measured, $4.3 \mathrm{~mm}$ posterior to bregma (sagittal axis). Along this sagittal axis, cortical thinning was again greatest in the stroke hemisphere, but also occurred in the intact hemisphere. Thinning was greatest proximal and immediately caudal to the infarct, but extended to distal residual tissue as well.

The statistical analysis confirmed that the cortex was thinner in the stroke 


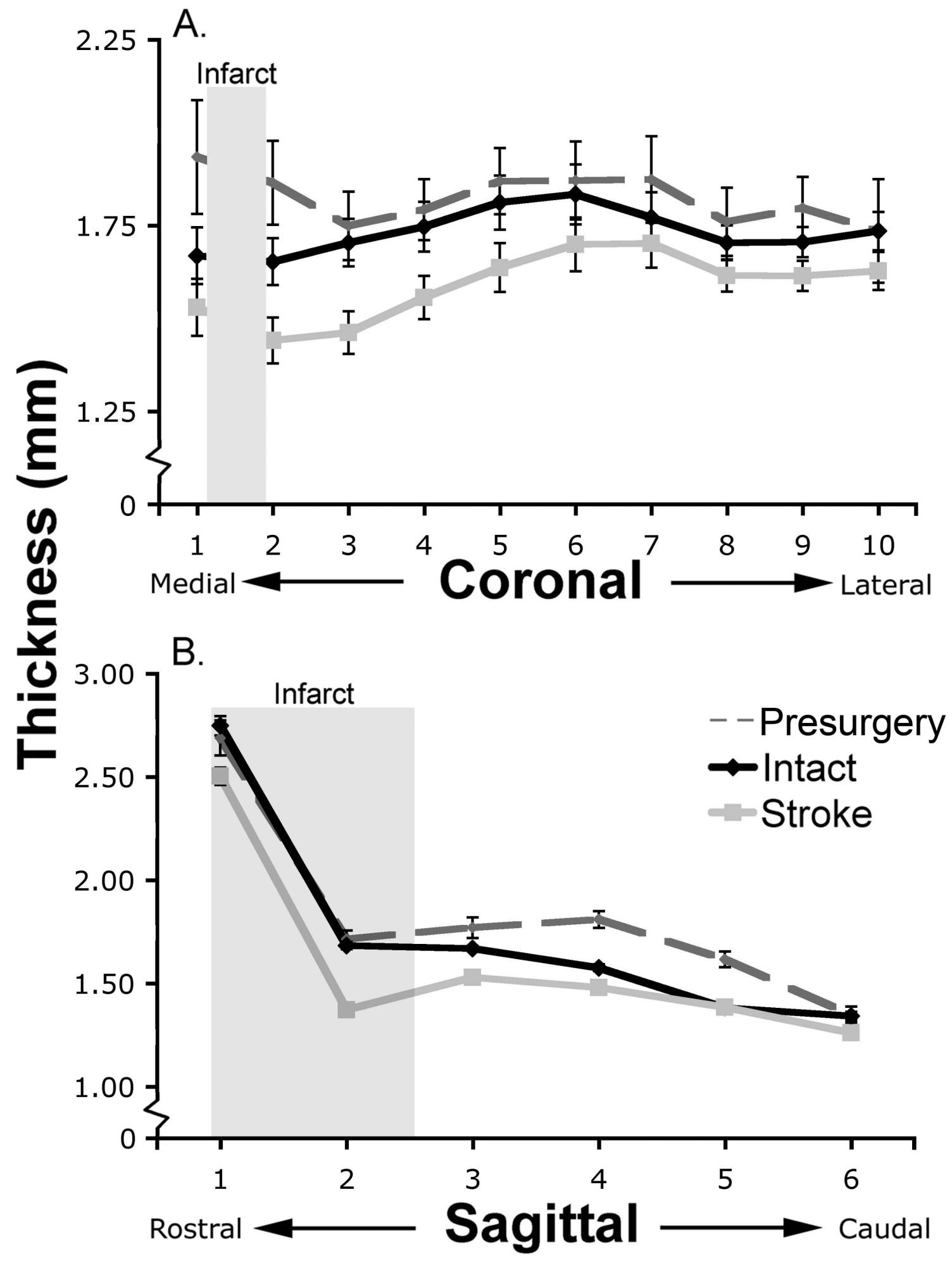


Fig. 17. Cortical thickness (mean and standard error) of coronal (A) and sagittal (B) points of measurement from rats pre-surgery (dotted line, $n=4$ ), the intact hemisphere of stroke rats (black, $n=4)$, and the stroke hemisphere of stroke rats (gray, $n=4)$ in the chronic time period. Note: Reductions in cortical thickness were greatest proximal to the stroke infarct but also extended to distal cortical tissue. (Shading represents histological planes containing the infarct). 
condition than in the pre-surgery condition and that the stroke hemisphere was thinner than the intact hemisphere within the stroke condition, HEMISPHERE X MEASURE $[\mathrm{F}(18,198)=5.583, \mathrm{p}<0.001]$ and HEMISPHERE X SECTION $[\mathrm{F}(10,110)=4.969$, $\mathrm{p}<0.001]$

\section{Residual Tissue Movement in Histological and MRI Sections}

Figure 18 (top) shows the location of lesion tracts, which served as anatomical markers of cortical tissue location, 31D post-stroke. Figure 18A shows the location of the lesion tracts (indicated by black arrows) in histological tissue sections. In the stroke

hemisphere (right) the lesion tracts were found closer to the midline, or infarct, compared to the lesion tracts in the intact hemisphere (left).

Figure 18B shows the location of the lesion tracts (indicated by white arrows) in MRI sections. In the stroke hemisphere (right) the lesion tracts were found closer to the midline, or infarct, compared to the lesion tracts in the intact hemisphere (left).

Figure 18C (bottom) shows a representative cresyl violet section illustrating the distance between the boundary of the somatosensory and motor cortices (diagonal black lines) to the center of the brain (dashed black line) in the intact and stroke hemispheres in the chronic post-stroke period. Figure 18D shows that this distance is decreased in the stroke hemisphere by $\sim 25 \%$. This main finding was confirmed by an ANOVA that gave a significant effect of HEMISPHERE $[\mathrm{F}(1,8)=33.43, \mathrm{p}<0.001]$. 
A.
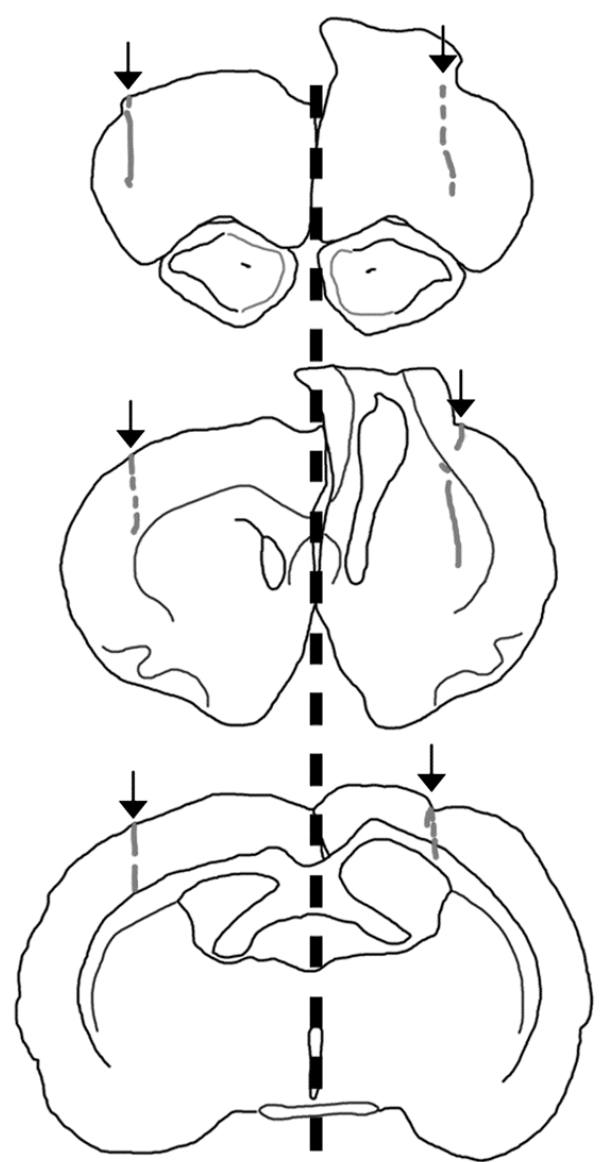

Intact Stroke

B.

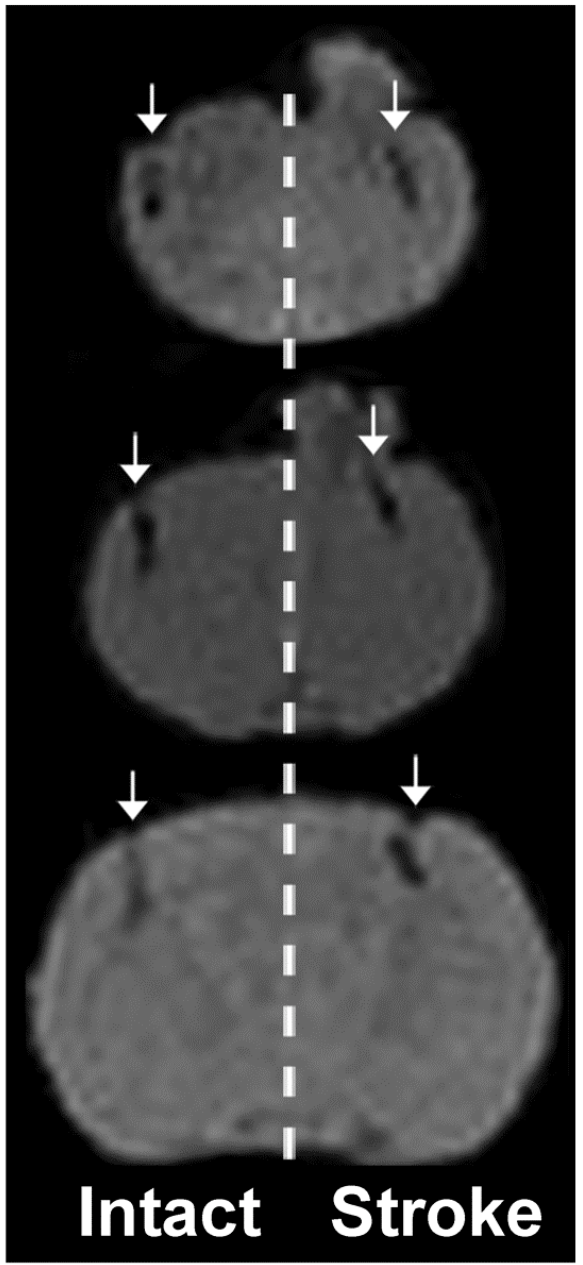

C.

D.

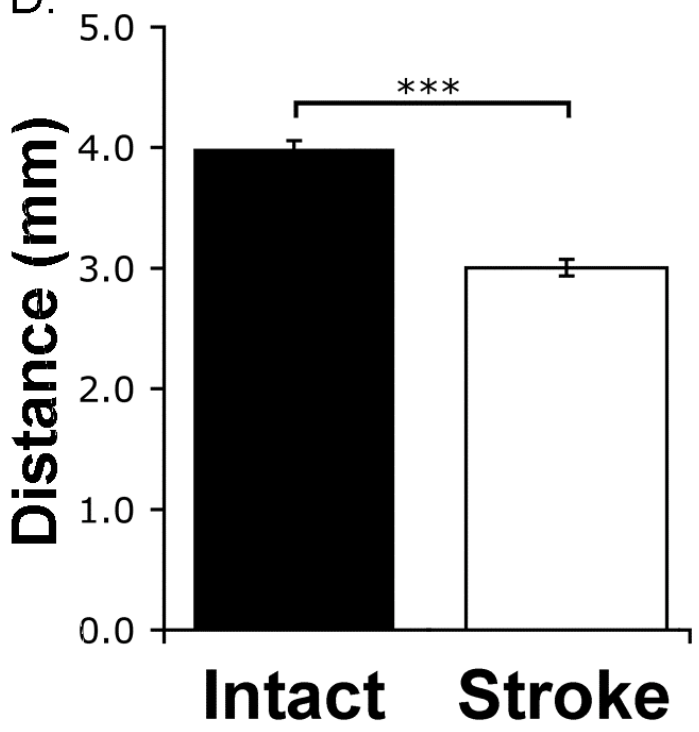


Fig. 18. Top. The location of lesion tracts (lines and arrows) in the stroke hemisphere (right) and intact hemisphere (left) at 31D after pial strip stroke are shown. A. Line tracings of coronal sections. B. MR images of coronal sections. Bottom. C. Representative cresyl violet section showing the distance between the darkly stained pyramidal cells in the somatosensory cortex (lateral to the diagonal black lines) and the midline of brain. Dashed lines indicate the brain midline. D. The distance (mean and standard error) between pyramidal cells in the somatosensory cortex and the brain midline in the intact $(n=9)$ and stroke $(n=9)$ hemispheres in the chronic post-stroke period. Note: the lesion tracts in the stroke hemisphere shifted toward the midline relative to the lesion tracts in the intact hemisphere, indicating that residual cortical tissue can move into the stroke infarct $31 \mathrm{D}$ after stroke in the adult rat. In addition, cytoarchitectural analyses show that pyramidal cells in the somatosensory cortex of the stroke hemisphere shifted $\sim 25 \%$ closer to the midline (or stroke) infarct relative to the intact hemisphere in the chronic post-stroke time period. (D - Day; MR - Magnetic Resonance: The dark region of the lesion tracts is not a cavity but is due to change in tissue perfusion density produced by the cannula insertions). 
In summary these results suggest that intact tissue in the stroke hemisphere shifted medially towards the center of the brain and into the stroke infarct. These results again indicate that cortical areas beyond the stroke infarct are susceptible to anatomical changes at chronic post-stroke time periods. These changes include extension of intact tissue into the stroke core, which could contribute to thinning of distal residual tissue by stretching it, and/or by aggravating secondary neuronal degeneration processes known to occur after focal cortical brain injury (Chen et al., 2003).

\section{Discussion}

In order to investigate the extent of peri-infarct tissue movement in a rodent model of focal cortical stroke, the movement of cortical tissue markers across a 1 month, post-stroke time period was studied using MRI. It was expected that if the formation of a cortical infarct is necessary for residual cortical thinning to occur (Karl et al., 2010), cortical thickness losses in proximal and distal residual tissue could be related to movement and stretching of peri-infarct tissue into the developing stroke infarct. Adult rats received unilateral, pial strip stroke of the motor cortex as well as temporary cannula insertions into intact surrounding cortical tissue (to act as markers of tissue location) and underwent serial MRI from 1 hour to 31 days post-stroke. Despite artificial tissue dehydration and the morphological anomalies that occur during histological processing, in vivo MRI changes in residual rat brain morphology were comparable to histological findings reported in previous studies (Karl et al., 2010). Decreases in cortical thickness were greatest in proximal peri-infarct tissue, the stroke hemisphere, and the chronic time 
period. In addition, residual tissue distal to the stroke core moved towards the infarct. These results suggest that tissue movement may be causally linked to post-stroke cortical thinning as well as the normalization of other aberrant peri-infarct MRI signals in the chronic post-stroke time period.

Although the extent of residual tissue thinning was comparable in the chronic time period between MRI and previously reported histological tissue sections, there was a difference in the acute time period. Cortical thickness in the stroke and intact hemispheres actually increased in vivo over the acute time period. It is likely that immediately after pial strip devascularization surgery the brain may become dehydrated due to exposure; this likely accounts for the immediate drop in cortical thickness observed at the $1 \mathrm{H}$ time point. Subsequent increases in cortical thickness are likely due to in vivo tissue edema. It has been found that the expression of aquaporin-4, a water channel protein that facilitates water diffusion into cells, is upregulated in the stroke infarct and penumbra in the acute post-stroke time period (Ribeiro et al., 2006). Thus, in vivo tissue swelling may have been apparent on MRI images, whereas artificial tissue dehydration, which occurs during histological processing, may have eliminated these effects in histological tissue. Nevertheless, changes in cortical thickness beyond the 1 hour time point were comparable in both histological and MRI sections.

One challenge in comparing pre-stroke neuronal tissue and post-stroke changes is in identifying homologous tissue. It is difficult to correctly identify peri-infarct tissue if it changes shape, size, and location. Furthermore, in studies of cortical changes in response to neonatal stroke, it has proven difficult to differentiate between intact tissue that has shifted to "fill in" the stroke infarct vs. new tissue that has arisen from post-injury 
neurogenesis (Kolb et al., 2007; Kuge et al., 2009; Shin et al., 2008). In the present study tissue was marked by a lesion, produced by temporary cannula insertions, and tissue changes were followed relative to the lesion tract using MRI. The results highlight the extent of cortical stretching, movement, and filling in of the stroke infarct. These findings are supported by subsequent analyses that confirmed that discrete cytoarchitectural elements in residual cortical tissue (ie. the darkly stained pyramidal cells of the somatosensory cortex) shifted towards the stroke infarct in the chronic time period. Other work using electrophysiological techniques also finds that peri-infarct tissue moves relative to the infarct location. (Eysel and Schweigart, 1999; Frost et al., 2003; Jenkins and Merzenich, 1987; Reinecke et al., 2003; Schiene et al., 1999; Zepeda et al., 2004).

Future studies of cytoarchitectural changes after focal cortical stroke could be used to quantify the full extent of tissue movement. Nevertheless, changes in peri-infarct tissue shape, size, and location do occur in the chronic post-stroke period. This could lead to erroneous comparison of control and peri-infarct tissue, and thus, morphological, electrophysiological, and molecular descriptions of peri-infarct cortex and its function must account for these gross anatomical changes in residual tissue after stroke.

A number of factors may have contributed to movement of residual tissue into the stroke infarct. For the present study, the infarct was located within the thickest cortical region (Fig. 19). Thus, removal of this tissue may lead to a reduction in gross structural support for surrounding tissue, which may then "fall" into the infarct. Secondly, processes of brain edema and inflammation may promote "swelling" of surrounding tissue such that it overflows into the infarct. Finally, edema and inflammation may 


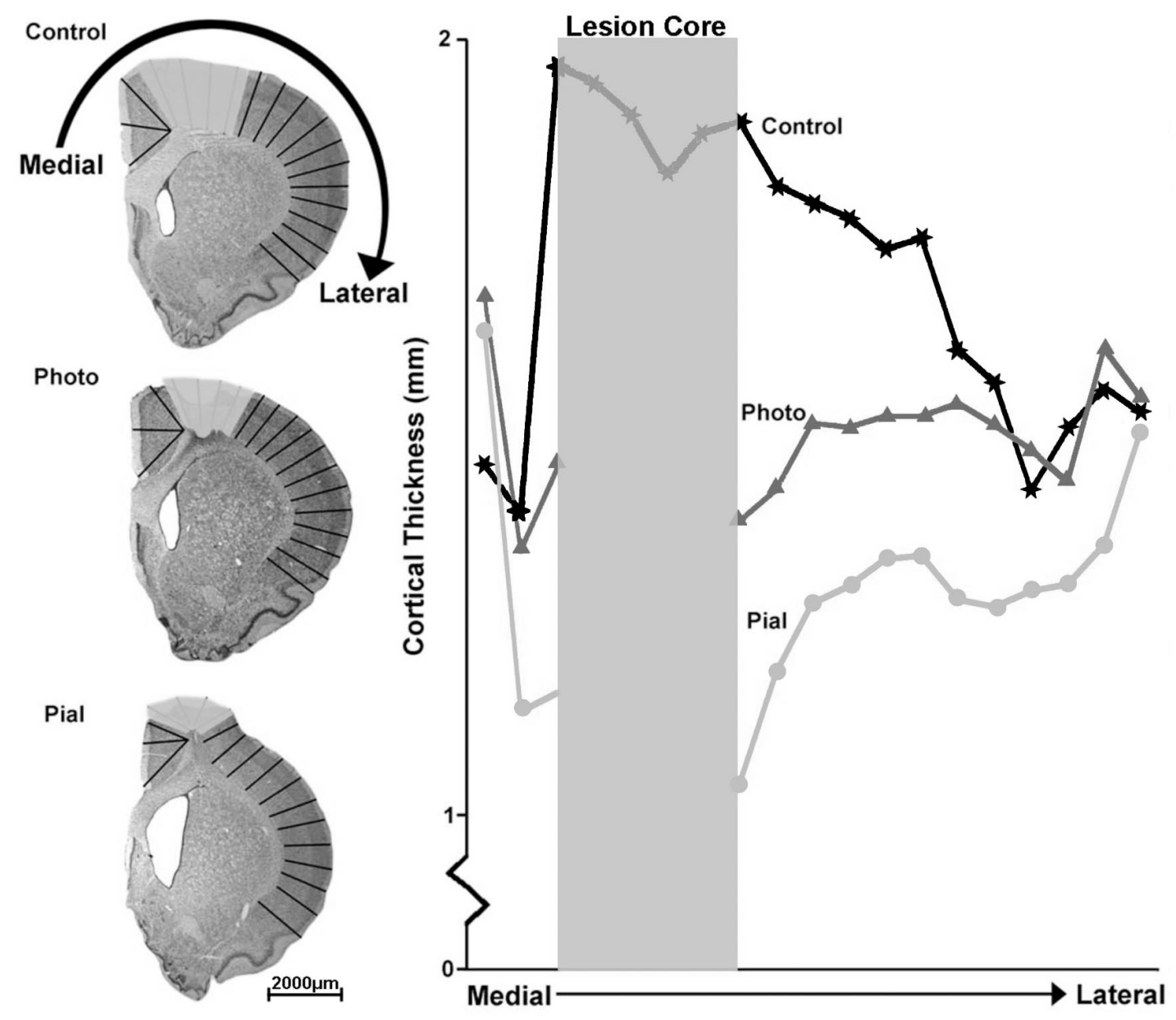


Fig. 19. Left: Measures of cortical thickness from one section of a control, photothrombotic, and pial strip animal (cresyl violet, 31 days post-stroke). Yellow areas indicate the location of the lesion core. Right: cortical thickness in the control (blue), photothrombotic (green), and pial strip (red) models. (Photo $=$ Photothrombotic, Pial $=$ Pial Strip). Note: When the motor cortex is targeted for focal cortical stroke the stroke infarct is located within the thickest region of cortex. 
amplify tissue movement processes in the pial strip devascularization model as residual tissue may swell into not only the infarct, but the overlying trephination as well. Preliminary pilot studies from our lab suggest that tissue movement also occurs after photothrombotic stroke of the motor cortex, a closed-head model, but to a lesser degree compared to pial strip devascularization (Fig. 20).

Previous magnetic resonance imaging (MRI) studies have shown time-dependent modification in stroke border zone tissue including T2 and fractional diffusion anisotropy (FA) normalization (van der Zijden et al., 2008a). It has been proposed that these alterations may be indicative of edema resolution, glial proliferation, and possibly white matter reorganization or restoration of neuronal connectivity which could lead to resolution of stroke-induced behavioural deficits (van der Zijden et al., 2008b). It is also possible however, that the restoration of MRI signals of peri-infarct tissue may be due to surrounding "intact" tissue extending into the stroke infarct and behavioural recovery may actually develop over time as the animal acquires compensatory behavioural strategies utilizing intact neural tissue.

The present results indicate that post-stroke tissue movement is not isolated to tissue immediately proximal to the infarct. Rather, substantial movement occurred in "intact" tissue significantly caudal to the stroke infarct. Thus, tissue movement may contribute to post-stroke thinning of distal cortical tissue by stretching distal corticocortical axons beyond a biologically viable threshold. It is interesting to consider that pathological processes in post-stroke residual tissue, such as vascular, glial, transcriptional, translational, electrophysiological, and apoptotic changes; as well as 

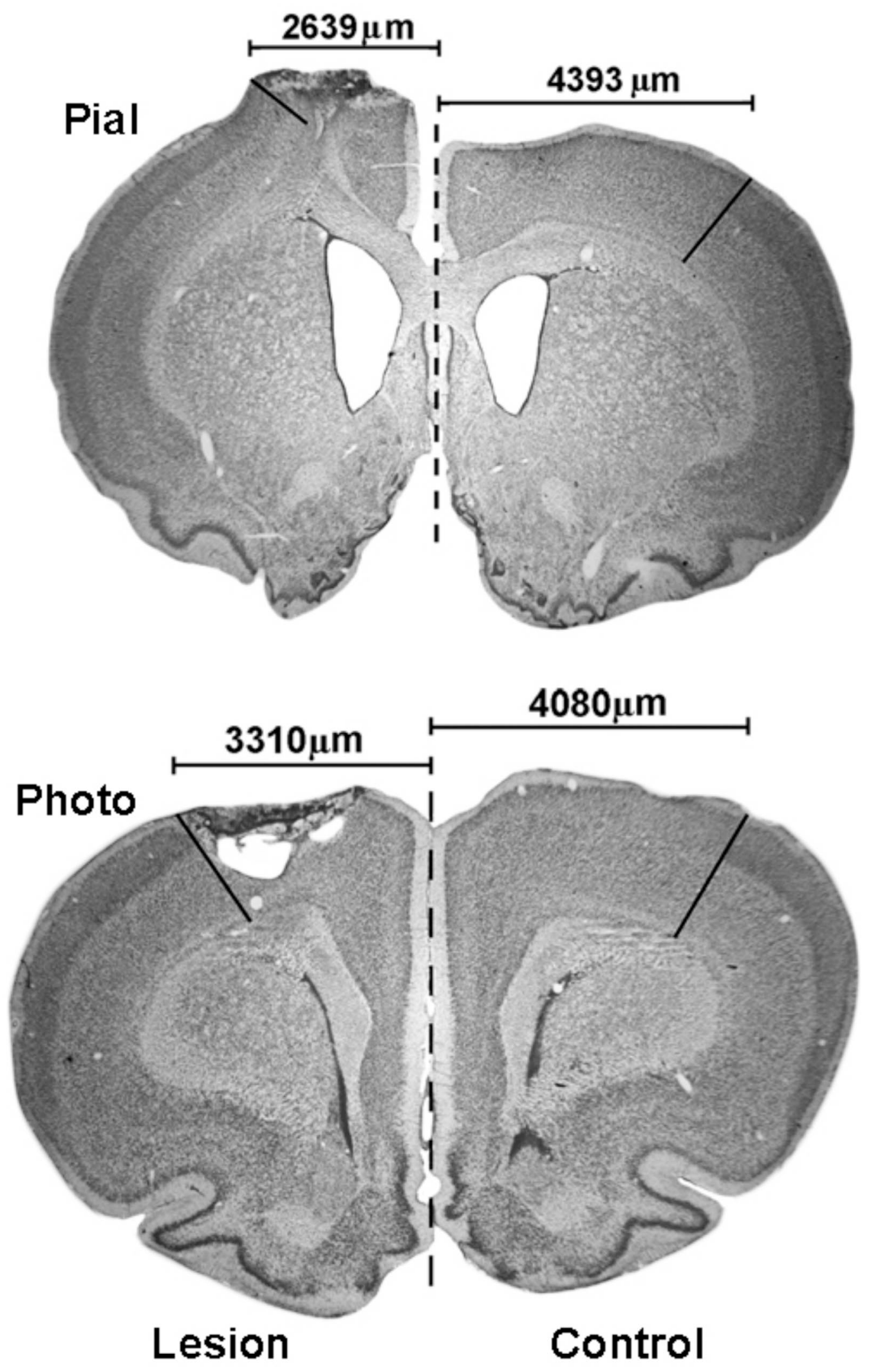
Fig. 20. Coronal sections from a representative pial strip and photothrombotic rat (cresyl violet, 31 days post-stroke). In each hemisphere a black line was drawn from the dorsal edge of the corpus callosum to the outer edge of the cortex such that it intersected the boundary between the darkly stained pyramidal cells in the somatosensory cortex (lateral to the black line) and the lighter stained pyramidal cells of the motor cortex (medial to the black line). The distance from this location to the midline of the brain is indicated above. Note: Pyramidal cells in the somatosensory cortex are located closer to the midline in the lesion hemisphere, compared to the intact hemisphere, in both stroke models. 
processes thought to mediate compensation and recovery in residual tissue such as axonal and dendritic sprouting may actually develop in response to thinning/stretching of the residual tissue in which they occur, rather than in response to tissue loss within the stroke infarct. Nevertheless, despite the gross morphological changes that take place distal to the stroke core this tissue apparently not only continues to subserve its normal behavioral functions, but can also mediate post-stroke behavioral compensation. 


\title{
CHAPTER FIVE \\ GENERAL DISCUSSION
}

\begin{abstract}
The analysis of gross residual cortical morphology after stroke in the adult rat has revealed that several important morphological changes occur in both proximal (penumbral) and distal peri-infarct tissue. Residual cortical thickness, volume, and density all decrease in the chronic post-stroke period. These effects are greatest in proximal peri-infarct tissue, but extend to distal cortical tissue and the intact hemisphere. The formation of a focal cortical infarct is necessary to induce thinning of residual cortical tissue, independent of the method of stroke induction or size. A stroke infarct localized to subcortical structures does not lead to thinning of the overlaying cortex. Movement of residual cortical tissue into the stroke infarct is an important feature of post-stroke morphological change in the adult rat and could be causally linked to poststroke decreases in cortical thickness, volume, and density. In the following discussion I first consider the contribution of these findings to the present literature. Secondly, the limitations of the present thesis will be described. In the third section the establishment of a new "plastic" model of residual cortical morphology in the adult rat, based on the evidence provided by the present thesis, will be discussed. The implications of this new "plastic" model for future stroke research will be considered in the fourth section. I conclude by considering the contribution of this new "plastic" model to current neuroscientific theory and understanding.
\end{abstract}




\section{Relevance to Present Literature}

The results of the present study are similar to those found after neonatal cortical stroke (Kolb and Holmes, 1983) and adds to current literature by describing the temporal and spatial progression of post-stroke cortical thinning in adult rat models. Additionally, by utilizing MRI, the present study is the first to confirm this morphological progression in vivo in an animal model and, to our knowledge, is the first to quantify the extent of residual tissue movement into the stroke infarct.

The present study differs from previous work in that the majority of previous studies found extensive cortical thinning after neonatal, but not adult stroke. There are a number of factors that may have contributed to this difference. One major difference is the methodology used to measure cortical thickness in past and present work. In the studies of Kolb and colleagues $(1983 a, b, 1985,1987)$ an average of 15 measures of cortical thickness were taken from each hemisphere ( 3 measures per section, 5 sections per rat). In the present study, an average of 100 cortical thickness measures were taken from each hemisphere (10 measures per section, 10 sections per rat). It is possible that a less rigorous measuring method may have missed changes in cortical thickness in the adult rat, especially if changes in the adult rat are less robust than in the neonatal rat.

Factors that could lead to more robust thinning after neonatal stroke include: 1) The use of cryoanesthesia when operating on neonatal rats. Cryoanesthesia has been shown to reduce cortical thickness in otherwise normal P1 rats (Kolb and Cioe, 2001) and could lead to increased cortical thinning in rats sustaining neonatal stroke vs. rats that 
receive stroke in adulthood, 2) Both degenerative and disrupted developmental mechanisms are likely to contribute to cortical thinning after neonatal stroke. The additional disruption of neurogenesis and neuronal migration/maturation after neonatal stroke may lead to greater reductions in cortical thickness, whereas cortical thinning in adult rats is more likely related to secondary neuronal degeneration processes, and 3) The length of time to sacrifice after stroke may lead to differences in residual cortical morphology. For instance, in many of the studies by Kolb and colleagues the neonatal operates were often not sacrificed until adulthood, 4 or more months after stroke induction. The adult operated rats were sacrificed at the same age despite the time from stroke induction being much shorter (1-2 months). As we have found in the present study that residual cortical thinning after stroke is a time-dependent phenomenon with the majority of thinning occurring in the chronic time period, it is possible that because the neonates survived for a longer post-stroke time they may have had additional thinning compared to the adult operates. Furthermore, because movement of tissue into the stroke infarct possibly contributes to residual tissue thinning, it is likely that extension of the chronic post-stroke time period for neonatal rats allowed more time for tissue to move into the stroke infarct, resulting in greater cortical thinning. Indeed, many studies of residual cortical morphology after neonatal stroke indicate greater "filling in of the stroke core" compared to adult operated rats, even when the stroke is induced at time periods when post-injury neurogenesis is not thought to contribute to the filling of the stroke infarct (Kolb et al., 1998).

In summary, the use of cryoanesthesia, the double hit of secondary neuronal degeneration and disrupted developmental processes, and the extension of the chronic 
time period after neonatal stroke may contribute to increased cortical thinning after neonatal stroke compared to adult stroke. Fewer measures of cortical thickness used in earlier studies may have been able to identify thinning after neonatal stroke, while missing thinning after adult stroke.

\section{Limitations of the Present Thesis}

This thesis presents with at least three limitations, which include the lack of a comparison of homologous tissue between the control, intact, and stroke hemispheres, gaps in the experimental timeline, and a potentially premature experimental duration. These limitations are discussed in detail below.

The major limitation of this thesis is that, due to the nature of the measuring method, it cannot be certain that comparisons between the control, intact, and stroke hemispheres were of homologous tissue. This is because the locations at which cortical thickness was measured were evenly distributed throughout residual tissue and did not take tissue movement into consideration. From the present results, it is now known that residual tissue can move towards the stroke infarct and could result in the comparison of non-homologous tissue between stroke and non-stroke brains, despite the sampled tissue being located at identical stereotaxic coordinates. One way to overcome this limitation is to compare cortical thickness, not equal distances from the stroke infarct, but in tissue with similar cytoarchitecture. This approach would reveal whether specific functional regions of the cortex are more susceptible than others to post-stroke cortical thinning. 
A second limitation of the present thesis is related to the time points at which cortical thickness was examined. The greatest change in cortical thickness was observed between 3 and 7 days post-stroke. This may represent a critical time period during which the extent of post-stroke morphological change may be influenced by internal and external factors. This time period was not studied in detail in the present thesis. Future investigations aimed at narrowing the size of this critical time period would be informative for developing strategies to alter the eventual extent of post-stroke morphological change.

Related to the time points of investigation is the overall length of the study. Animals were culled at a maximum of 31 days after stroke. However, results from the present thesis suggest that morphological change may continue beyond that point. Molecular changes in distal residual tissue are known to persist for months after the formation of an infarct (Nakano et al., 1990) and it is possible that tissue thinning and movement may as well. The present thesis is unable to address the nature of morphological change that occurs beyond 31 days. Extension of post-stroke survival time would also allow us to investigate the interaction of pathological cortical thinning and cortical thinning associated with normal aging (Hughes and Lantos, 1987). It has been suggested that brain insults, such as stroke, may speed and/or alter the normal process of brain aging.

A final limitation of the present thesis is the use of Grey Value Indices (GVI) as a measure of tissue density. A nissl stain, which localizes in ribosomes, was used for GVI analyses. Ribosomes mediate translation of RNA to protein. After stroke, large changes in protein production are known to occur in ischemic, proximal, and distal residual tissue. 
Some proteins are upregulated, while others are downregulated. Whether there is a net change in overall protein production is not known. Nevertheless, as there may be changes in overall protein production after stroke, this could either increase or decrease the number of ribosomes present in the cell. This change may or may not alter the concentration of nissl stain in cell bodies. If a signifcant change in ribosome number and nissl stain concentration was found to occur after stroke this could influence the GVI measures, which may then not provide a consistent measure of cell density before and after stroke. At present, I am not aware of any study that has shown that the number of ribosomes is significantly different between ischemic and non-ischemic tissue.

Despite the aforementioned limitations, the present thesis successfully characterizes the general nature and extent of morphological change up to 1 month after cortical stroke in the adult rat. A large number of preclinical studies investigate the function of residual tissue within this time period; thus, consideration of these morphological changes will be an essential factor for improving our understanding of the contribution of residual tissue to functional recovery after stroke.

\section{A "Plastic" Model of Adult Rat Cortical Morphology after Stroke}

The results of the present thesis support the modification of the "topographical" model (Fig. 21A) of rat post-stroke cortical morphology in favor of a "plastic" model (Fig. 21B). The traditional "topographical" model is based on molecular, cellular, and electrophysiological properties of residual cortical tissue but lacks reference to the gross morphological context within which these properties exist. The "plastic" model 
acknowledges the dynamic morphological nature of residual cortical tissue. According to this model, penumbral and distal cortical tissue can stretch towards, and partially "fillin", the stroke infarct. This, in turn, modifies the location and organization of residual functional areas, re-defining their original borders. The interpretation of experimental findings based on both the microscopic and gross morphological properties of residual cortical tissue will provide the most comprehensive model for residual cortical tissue function.

\section{Theoretical Implications of the "Plastic" Model for Investigations of Peri-Infarct Tissue Function}

The plastic model of residual cortical morphology has a number of implications for preclinical stroke research. Below I will consider how stretching and thinning of residual cortex may influence the study and interpretation of molecular, cellular, and electrophysiological properties of residual cortex after stroke.

\section{Molecular Implications of the Plastic Model}

As mentioned in Chapter 1, numerous molecular changes occur in residual cortex after stroke. These changes are often attributed either to pathological processes extending from the infarct core, or to plastic properties of intact "functional areas" that mediate compensation and recovery after stroke. According to the plastic model, it is also possible that these "changes" aren't really changes at all. It could be the case that a more distal 


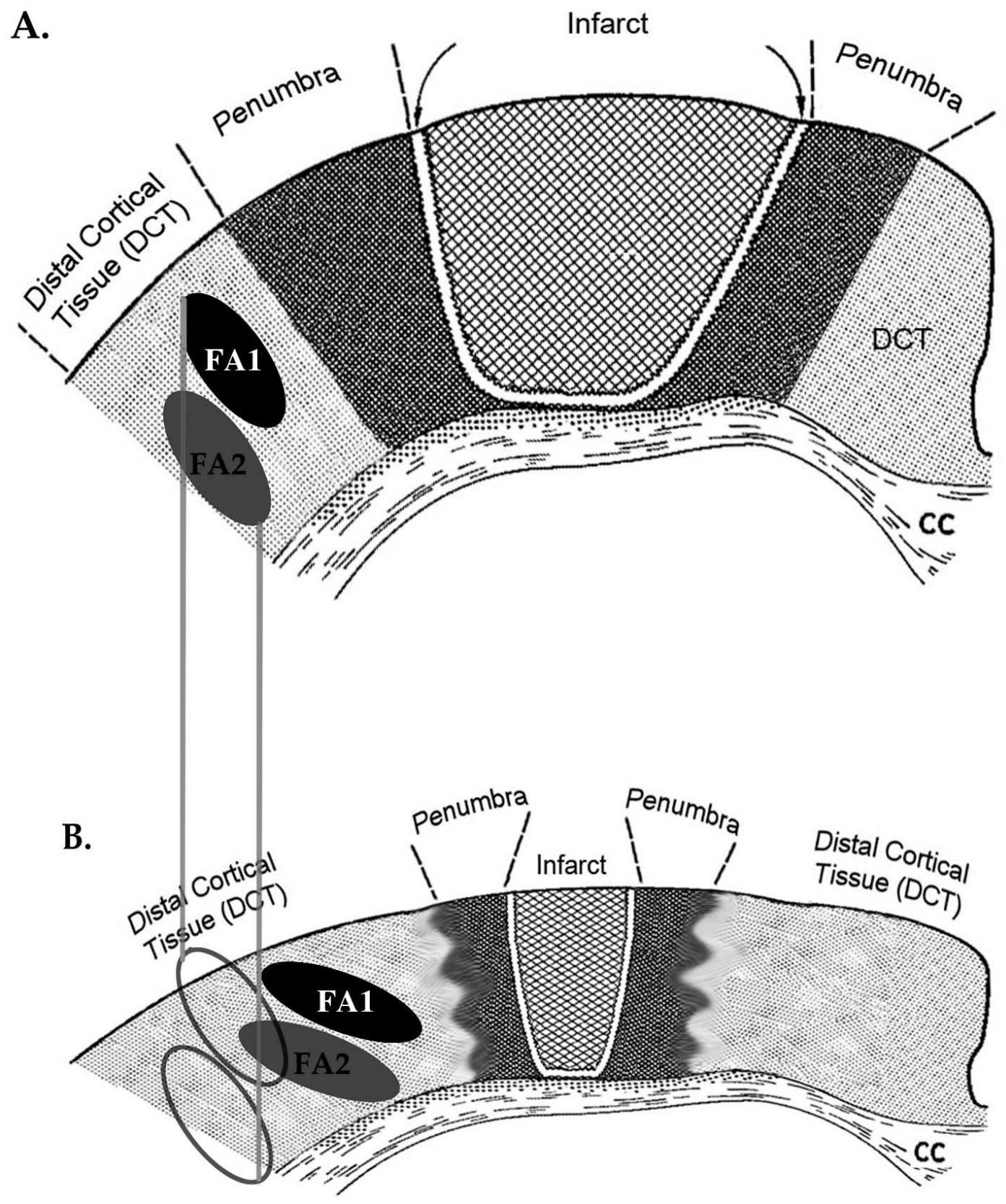


Figure 21. The traditional "topographical" model of residual cortical morphology in the adult rat (A, adapted from Bidmon et al., 1997). The new "plastic" model of residual cortical morphology in the adult rat (B). Note: The red lines and black circles indicate the expected location of functional areas 1 and 2 after stroke. Thinning and stretching of residual cortex in the plastic model leads to a shift in the shape and location of residual functional areas such that they are not found at their original stereotaxic coordinates. FA 1 - Function Area 1, FA 2 - Functional Area 2, DCT - Distal Cortical Tissue, CC Corpus Callosum 
functional area, let's say functional area 2, which has it's own distinct molecular nature, has simply shifted closer to the infarct, and into the space normally occupied by functional area 1. Without consideration of tissue thinning and movement, investigation at the original location of functional area 1, would lead to the erroneous attribution of novel molecular characteristics (characteristics normally attributed to functional area 2) to this area. This same error would also apply when investigating cellular, electrophysiological, and morphological characteristics of peri-infarct tissue.

One way of overcoming this challenge is to demark functional cortical areas of interest prior to stroke induction. This may be achieved using a number of anterograde or retrograde tract tracing dyes, direct injection of cellular/nuclear dyes, or physical demarcation, as was used in Chapter 4 of this thesis. Using these techniques, post-mortem histological analysis would confirm the location of the tissue of interest and whether or not it was correctly identified during post-stroke investigations of residual tissue.

As indicated above, molecular changes in residual tissue could be attributed to a pathological response to cell death in the stroke infarct, plastic processes in intact tissue that mediate compensation and recovery, or movement of distal cortical tissue closer to the stroke infarct. Another possibility exists; molecular changes in distal cortical tissue could indicate a response to pathological processes, not originating in the infarct, but within the distal residual tissue itself. As discussed in Chapter 4, stretching of axons in residual cortical tissue could lead to cellular stress, inflammation, and death in distal residual tissue. Thus, changes in protein synthesis and neurotrophic factor expression in distal cortical areas could be the cell's strategy for managing these noxious events and limiting damage to distal residual tissue from these secondary insults. 
Future experimentation could determine the extent of axonal damage caused by tissue stretching by testing $68 \mathrm{kDa}$ neurofilament immunoreactivity, a marker of axonal injury (Yam et al., 1998), after stroke. A marker of neuronal death, such as fluoro jade-B (Schmued and Hopkins, 2000), could also be used to determine the temporal and spatial pattern of secondary neuronal degeneration in residual tissue and whether or not it parallels axonal damage.

\section{Cellular Implications of the Plastic Model}

According to the plastic model, residual tissue thinning and movement could directly alter the cytoarchitecture of residual tissue. In the following section the implications for neuronal processes, the cell body, and distinct cellular groupings within thinned/stretched residual tissue will be considered.

Firstly, one can ask, what happens to the morphology of neuronal processes when the gross morphology of the cortex changes? If the cells stretch (along with the cortex), this could lead to the elongation of lateral dendrites. This could inadvertently decrease spine and/or synapse density along the mediolateral and rostrocaudal planes. What happens if the cells are compressed along the dorsoventral plane (along with thinning of the cortex)? This could theoretically lead to the compression of apical (dorsal/ventral) dendrites. This could inadvertently increase spine and/or synapse density along the dorsoventral plane. Both histological examinations, using golgi-cox staining, and in vivo cellular imaging methods (Brown and Murphy, 2008) could be used to correlate the extent of tissue thinning/stretching with neurite changes in residual cortex. 
In the present thesis, cellular density decreased in residual tissue. Two possibilities exist, additional cells in peri-infarct tissue may have died, or they may have shrunk. Future studies using stereological methods to count individual cells in peri-infarct tissue could answer this question.

One way that cells are grouped in the cortex is in a laminar formation. It is possible that some cortical lamina are more susceptible to thinning than others, this could be tested by measuring the thickness of individual cortical layers in the chronic poststroke time period. If some layers are proportionally thinner than others this may have functional implications. Cortical cells are also grouped into columns and different functional areas (as mentioned above); residual tissue thinning and movement could also differentially affect these cellular groupings.

Consequently, application of the plastic model suggests that cytoarchitectural changes in residual tissue may indicate not only a pathological or compensatory/plastic response to the stroke, but also an indirect response to thinning and movement of the tissue within which they are located.

\section{Electrophysiological Implications of the Plastic Model}

The plastic model of rat cortex after stroke also implies that electrophysiological properties of residual cortex may be subjective to residual tissue thinning and movement. Cortical forelimb representations, as determined by intracortical microstimulation (ICMS), are sensitive to motor cortex function and motor ability. The size and organization of the "motor map" is altered in response to, and is thus indicative of, 
cortical injury (Gharbawie et al., 2005; Nudo, 1997; Williams et al., 2006), corticofugal fiber function (Kartje-Tillotson et al., 1987), and motor experience/learning (Adkins et al., 2006; Jacobs and Donoghue, 1991; Kleim et al., 1998, 2002). Changes in ICMS motor map organization are also often used as a measure of peri-infarct tissue function after focal cortical stroke (Gharbawie et al., 2005).

ICMS requires that a stimulating electrode be lowered to a depth of $1550 \mu \mathrm{m}$ to stimulate layer V pyramidal cells (Gharbawie et al., 2005; Karl et al., 2008; Kleim et al., 2004; Remple et al., 2001; Williams et al., 2006). Cortical thinning could cause layer V pyramidal cells to shift along the dorsoventral axis after stroke. Consequently, the dorsoventral location of the stimulating electrode may need to be adjusted when examining peri-infarct tissue in a stroke animal compared to controls. Failure to do so could result in an erroneous interpretation of the size of residual cortical maps after stroke.

Movement of cortical tissue along the mediolateral or rostrocaudal planes will likely alter the location of homologous tissue in the stroke, intact, and control hemispheres, such that they are not found at identical stereotaxic coordinates. Previous studies have found that cortical motor maps can expand after focal cortical stroke (Williams et al., 2006). According to the plastic model, it is possible that map expansion could be at least partially due to stretching of residual tissue, rather then compensatory/plastic expansion of intact neural circuits.

\section{Morphological Implications of the Plastic Model}


The plastic model also indicates that smaller morphological changes in residual cortical tissue are likely to be compromised by larger morphological changes within the same tissue. Many investigations of peri-infarct tissue utilize the intact, contralateral hemisphere as a control. The present thesis indicates that post-stroke morphological change is not isolated to the stroke hemisphere but extends to the intact hemisphere as well. Previous work also suggests that the morphology of the intact contralateral sensorimotor cortex is especially susceptible to change after unilateral damage to the opposite sensorimotor cortex (Allred and Jones, 2004; Hsu and Jones, 2006). Thus, to determine absolute changes in ipsilateral, peri-infarct tissue morphology, the proper control tissue must come from a healthy control animal, not the intact, contralateral hemisphere, as this could lead to an underestimation of the actual degree of change in the stroke hemisphere.

Other investigations have suggested that proximal peri-infarct tissue can actually thicken after stroke (Bidmon et al., 1997). In this study, peri-infarct tissue thickness was investigated in isolation, without consideration for morphological change occurring in more distal residual tissue. According to the plastic model, it is possible that the "thickening" observed in this study could be due to the movement of naturally thicker cortex from surrounding cortical areas closer to the stroke infarct. Interestingly, the authors of this study also indicate that, in the chronic time period (after 30 days), “...the [cortical thickness] values then became negative for the frontal and parietal perilesional cortices, due to the shrinkage of the core and a partial closure of the scar by the perilesional cortex." 


\section{Contribution of the "Plastic" Model to Current Neuroscientific Theory}

An understanding of the gross morphological changes that occur in the residual cortex presents a number of insights about general brain function. In the following sections I will discuss how the plastic model of residual cortical morphology can improve our understanding of localization of function, diaschisis (temporary disconnection syndromes), and behavioural compensation after stroke.

\section{Localization of Function}

The results of the present study indicate that morphological changes in residual cortex are more widespread than originally thought. One major theory that considers the possibility that a focal cortical infarct could induce widespread dysfunction is Karl Lashley's "Principle of Mass Action".

Lashley proposed that functions within the cortex are not localized, but rather the cerebral cortex acts as a whole to perform numerous and varied functions (Lashely, 1931). He suggested that the specific location of a cortical injury does not influence the nature of the animal's functional outcome, but rather the larger the injury the larger the post-stroke functional deficit. In addition, the more complex the behaviour, the more sensitive it is to cortical injury. Lashley came to his conclusions after decades of systematically varying the size and location of cerebral infarcts in rats followed by thorough behavioural testing. Although the present thesis did not directly correlate 
behaviour with post-stroke morphological change, the morphological results may have significant implications for this theory.

The results of this thesis indicate that the physical consequences of a localized injury are not localized in and of themselves. Thinning and movement of residual tissue can occur at great distances from the stroke core, affecting widespread areas of "normal" cortical tissue. Furthermore, the present results suggest that the larger the size of injury, the larger the extent of cortical thinning in residual cortical tissues. Thus, it is possible that widespread changes in gross morphology could disrupt the functioning of distal but functionally localized tissue to produce generalized deficits after a "localized" lesion. Thus, Lashley may have been correct in that "localized lesions" can produce generalized behavioural impairments. His conclusion that the cortex must then participate in "mass action" however, may be compromised, as it is possible that widespread morphological changes could also have widespread effects on tissue function. Stretching of residual cortical tissue into the infarct could be one mechanism by which functioning of distal cortical areas could be compromised.

\section{Disconnection}

Wallerian degeneration of axons distal to cell bodies located within the stroke infarct, and the subsequent degeneration of cells that synapse with atrophied neurons is a known mechanism of secondary tissue degeneration after brain injury (Coleman and Perry, 2002; Enright et al., 2007; Iizuka et al., 1989; Kuhn et al., 1989; Pujol et al., 1990; Sawlani et al., 1997; Sigler et al., 2009; Waller, 1850). 
It is possible that the effects of Wallerian degeneration may be enhanced in cortical tissue that lacks gross structural support and shifts into a developing cortical infarct. This shift could force the elongation of already affected cortico-cortical and cortico-subcortical axons beyond the biological threshold for Wallerian degeneration, subsequently inducing axonal and neuronal atrophy at increasingly distal and diffuse cortical and subcortical sites. This, in turn, could amplify edema, gliosis, molecular, physiological, and apoptotic responses in remote peri-infarct tissue.

Von Monakow (1914) proposed that cell death within the stroke infarct and subsequent Wallerian degeneration can lead to the disconnection and dysfunction of remote "intact" cortical areas efferent to the stroke infarct. This post-stroke disconnection, termed "diaschisis", could lead to generalized behavioural deficits after a localized injury. Diaschisis effects may be amplified in residual cortical tissue that stretches into a stroke infarct. The challenge of future investigations will be in determining which post-stroke functional deficits can be attributed to isolated cell death within the stroke infarct, versus general dysfunction of surrounding residual tissue undergoing various morphological, electrophysiological, and molecular changes.

\section{Behavioural Recovery/Compensation}

Because plastic processes in peri-infarct tissue mediate recovery after stroke, it is possible that stretching/thinning of remaining tissue will influence functional and behavioural recovery. Previous MRI studies in human stroke patients have found that the extent of Wallerian degeneration predicts behavioural recovery outcomes (Kuhn et al., 
1989; Sawlani et al., 1997). Additionally, protrusion of cortical tissue into the stroke infarct has been associated with impaired skilled motor recovery in rats. Whishaw (2000) found that following motor cortex stroke, rats that experience protrusion of surrounding cortical and subcortical tissue into the infarct perform worse and recover to a lesser extent on a skilled reaching for food task compared to rats with no, or only subcortical tissue protrusion into the infarct.

In humans, reduced cortical thickness has also been associated with OCD (Shin et al., 2007), schizophrenia (Kuperberg et al., 2003; Lawyer et al., 2008; Nesvag et al., 2008; Zipursky et al., 1992), amnesia (Seo et al., 2007: Salat et al., 2006), Huntington's disease (Rosas et al., 2002, 2005, 2008), Alzheimer's disease (Dickerson et al., 2008; Richards et al., 2008), epilepsy (Lee et al., 1995; McDonald et al., 2008), traumatic brain injury (Merkley et al., 2008) and normal ageing (Preul et al., 2006). In many of these cases the severity of disease symptoms and/or cognitive decline was related to the degree of cortical thinning.

It is noteworthy that most of the anatomical changes described in the present thesis took place in the chronic post-stroke period, a time at which behavioral work demonstrates that behavioral recovery is occurring and can be influenced by rehabilitative procedures. Possibly, rehabilitative success importantly depends upon a permissive environment created by the underlying post-stroke neuronal changes. Longterm behavioural treatments that improve functional recovery after stroke, such as environmental enrichment, have also been found to increase cortical thickness in normal and stroke animals (Comeau et al., 2008). Thus, although, the cause and effect 
relationship is still not fully understood, future studies aimed at maintaining residual cortical thickness may also contribute to behavioural and functional recovery after stroke.

\section{Conclusions}

Morphological change has been thoroughly described after neonatal stroke. The extent of morphological change following stroke in the adult rat, however, has been underestimated in the literature. This is evidenced by the lack of consideration given to cortical tissue thinning and movement, despite the potential impact on molecular, cellular, and electrophysiological properties of residual tissue.

The present thesis systematically describes tissue thinning, volume loss, density loss, and movement of residual tissue after stroke in the adult rat. In summary, cortical thinning in rodent models of stroke is a temporally and spatially dependent phenomenon. The greatest reductions in cortical thickness occur at a considerable time after stroke in not only peri-infarct tissue but also extend to distal cortical tissue including the intact hemisphere. Movement of residual tissue into the stroke infarct, degeneration of neuropile, and adaptive anatomical changes likely contribute to post-stroke tissue thinning.

These findings make three important contributions to current preclinical stroke research. Firstly, they provide a benchmark as to the extent of morphological change that can be expected after focal cortical stroke in the adult rat. Secondly, these findings indicate that microscopic changes in residual cortical tissue may occur in response to not only pathological or compensatory/plasticity processes, but also in response to 
thinning/stretching of the cortical tissue within which they occur. It is interesting that, despite the gross morphological changes that take place distal to the stroke infarct, this tissue apparently not only continues to subserve its normal behavioural functions, but can also adapt to mediate behavioural compensation. Finally, these findings support the transition from a "topographical" model of post-stroke cortical morphology, to a new, "plastic" model. It is our hope that researchers will use the plastic model to improve the validity of preclinical stroke research by accounting for residual tissue thinning and movement after stroke in the adult rat; and, by ensuring that homologous cortical tissue is compared between stroke and non-stroke animals. 


\section{REFERENCES}

Abrahams JM, Lenart CJ, Tobias ME (2009) Temporal variation of induction neurogenesis in a rat model of transient middle cerebral artery occlusion. Neurological Research, 31:528-533.

Adams FS, Schwaring RK, Huston JP (1994) Behavioral and neurochemical asymmetries following unilateral trephination of the rat skull: is this control operation always appropriate? Physiology \& Behavior, 55:947-952.

Adkins DL, Boychuk J, Remple MS, Kleim JA (2006) Motor training induces experience-specific patterns of plasticity across motor cortex and spinal cord. Journal of Applied Physiology, 101:1776-1782.

Alaverdashvili M, Moon SK, Beckman CD, Virag A, Whishaw IQ (2008) Acute but not chronic differences in skilled reaching for food following motor cortex devascularization vs. photothrombotic stroke in the rat. Neuroscience, 157:297-308.

Alaverdashvili M \& Whishaw IQ (2010) Compensation aids skilled reaching in aging and in recovery from forelimb motor cortex stroke in the rat. Neuroscience, 167:21-30.

Allred RP \& Jones TA (2004) Unilateral ischemic sensorimotor cortical damage in female rats: forelimb behavioral effects and dendritic structural plasticity in the contralateral homotopic cortex. Experimental Neurology, 190:433-445.

Back T, Ginsberg MD, Dietrich WD, Watson BD (1996) Induction of spreading depression in the ischemic hemisphere following experimental middle cerebral artery occlusion: Effect on infarct morphology. Journal of Cerebral Blood Flow and Metabolism, 16:202-213.

Bayer SA \& Altman J (1991) Neocortical development. New York: Raven Press.

Bidmon HJ, Jancisk V, Schleicher A, Hagemann G, Witte OW, Woodhams P, Zilles K (1998) Structural alterations and changes in cytoskeletal proteins and proteoglycans after focal cortical ischemia. Neuroscience, 397-420.

Biernaskie J \& Corbett D (2001) Enriched rehabilitative training promotes improved forelimb motor function and enhanced dendritic growth after focal ischemic injury. Journal of Neuroscience, 21:5272-5280.

Branston NM, Strong AJ, Symon L (1977) Extracellular potassium activity, evoked potential and tissue blood flow: relationships during progressive ischaemia in baboon cerebral cortex. Journal of the Neurological Sciences, 32:305-321. 
Brown CE, Boyd JD, Delaney KR, Murphy TH (2007) Extensive turnover of dendritic spines and vascular remodeling in cortical tissues recovering from stroke. Journal of Neuroscience, 27:4101-4109.

Brown CE \& Murphy TH (2008) Livin' on the edge: Imaging dendritic spine turnover in the peri-infarct zone during ischemic stroke and recovery. The Neuroscientist, 14:139146.

Brown CE, Wong C, Murphy TH (2008) Rapid morphological plasticity of peri-infarct dendritic spines after focal ischemic stroke. Stroke, 39:1286-1291.

Buchkremer-Ratzmann I, August M, Hagemann G, Witte OW (1996) Electrophysiological transcortical diaschisis after cortical photothrombosis in rat brain. Stroke, 27:1105-1109.

Buchkremer-Ratzmann I \& Witte OW (1996) Extended brain disinhibition following small photothrombotic lesions in rat frontal cortex. Neuroreport, 8:519-522.

Bury SD, Adkins DL, Ishida JT, Kotzer CM, Eichhorn AC, Jones TA (2000) Denervation facilitates neuronal growth in the motor cortex of rats in the presence of behavioral demands. Neuroscience Letters, 287:85-88.

Butefisch CM (2006) Neurological bases of rehabilitation. Neurological Sciences, 27:173-185.

Carmichael ST, Archibeque I, Luke L, Nolan T, Momiy J, Li S (2005) Growth-associated gene expression after stroke: evidence for a growth-promoting region in peri-infarct cortex. Experimental Neurology, 193:291-311.

Carmichael ST, Wei L, Rovainen CM, Woolsey TA (2001) New patterns of intracortical projections after focal cortical stroke. Neurobiology of Disease, 8:910-922.

Castro-Alamancos MA, Borrel J (1995) Functional recovery of forelimb response capacity after forelimb primary motor cortex damage in the rat is due to the reorganization of adjacent areas of cortex. Neuroscience 68:793-805.

Chen J, Graham SH, Chan PH, Lan J, Zhou RL, Simon RP (1995) Bcl-2 is expressed in neurons that survive focal ischemia in the rat. Neuroreport, 6:394-398.

Chen S, Pickard JD, Harris NG (2003) Time course of cellular pathology after controlled cortical impact injury. Experimental Neurology, 182:87-102.

Chiganos TC, Jensen W, Rousche PJ (2006) Electrophysiological response dynamics during focal cortical ischemia. Journal of Neural Engineering, 3:L15-L22. 
Chu CJ \& Jones TA (2000) Experience-dependent structural plasticity in cortex heterotopic to focal sensorimotor cortical damage. Experimental Neurology, 166:403414.

Coleman MP \& Perry VH (2002) Axon pathology in neurological disease: a neglected therapeutic target. Trends in Neuroscience, 25:532-537.

Comeau W, Gibb R, Hastings E, Cioe J, Kolb B (2008) Therapeutic effects of complex rearing of bFGF after perinatal frontal lesions. Developmental Psychobiology, 50:134146.

Dickerson BC, Bakkour A, Salat DH, Feczko E, Pacheco J, Greve DN, Grodstein F, Wright CI, Blacker D, Rosas HD, Sperling RA, Atri A, Growdon JH, Hyman BT, Morris JC, Fischl B, Buckner RL (2008) Cerebral Cortex, doi:10.1093/cercor/bhn113.

Dijkhuizen RM, Beekwilder JP, van der Worp HB, van der Sprenkel JWB, Tuleken KAF, Nicolay K (1999) Correlation between tissue depolarizations and damage in focal ischemic rat brain. Brain Research, 840:194-205.

Dirnagl U, Iadecola C, Moskowitz MA (1999) Pathobiology of ischaemic stroke: An integrated view. Trends in Neuroscienc, 22:391-397.

Donoghue JP \& Wise SP (1982) The motor cortex of the rat, cytoarchitecture and microstimulation mapping. The Journal of Comparative Neurology, 212:76-88.

Durukan A \& Tatlisumak T (2009) Ischemic stroke in mice and rats. Methods in Molecular Biology, 573:95-114.

Endres M, Fink K, Zhu J, Staliano NE, Bondada V, Geddes JW, Azuma T, Mattson MP, Kwiatkowski DJ, Moskowitz MA (1999) Neuroprotective effects of gelsolin during murine stroke. The Journal of Clinical Investigation, 103:347-354.

Enright LA, Zhang S, Murphy TH (2007) Fine mapping of the spatial relationship between acute ischemia and dendritic structure indicates selective vulnerability of layer $\mathrm{V}$ neuron dendritic tufts within single neurons in vivo. Journal of Cerebral Blood Flow \& Metabolism, 27:1185-1200.

Erickson CA, Gharbawie OA, Whishaw IQ (2007) Attempt-dependent decrease in skilled reaching characterizes the acute postsurgical period following a forelimb motor cortex lesion: an experimental demonstration of learned nonuse in the rat. Behavioural Brain Research, 179:208-218.

Eysel UT \& Schweigart G (1999) Increased receptive field size in the surround of chronic lesions in the adult cat visual cortex. Cerebral Cortex, 9:101-109. 
Ferrer I \& Planas AM (2003) Signaling of cell death and cell survival following focal cerebral ischemia: Life and death struggle in the penumbra. Journal of neuropathology and experimental neurology, 62:329-339.

Fridman EA, Hanakawa T, Chung M, Hummel F, Leiguarda RC, Cohen LG (2004) Reorganization of the human ipsilesional premotor cortex after stroke. Brain, 127:747758.

Friel KM, Heddings AA, Nudo RJ (2000) Effects of postlesion experience on behavioral recovery and neurophysiologic reorganization after cortical injury in primates. Neurorehabilitation and Neural Repair, 14:187-198.

Frontczak-Baniewicz M, Walski M (2006) Glial scar instability after brain injury. Journal of Physiology and Pharmacology, 57:97-102.

Frost SB, Barbay S, Friel KM, Plautz EJ, Nudo RJ (2003) Reorganization of remote cortical regions after ischemic brain injury: a potential substrate for stroke recovery. Journal of Neurophysiology, 89:3205-3214.

Garman RH (1990) Artifacts in routinely immersion fixed nervous tissue. Toxicological Pathology, 18:149-153.

Garcia JH, Liu KF, Ho KL (1995) Neuronal necrosis after middle cerebral artery occlusion in Wistar rats progresses at different time intervals in the caudoputamen and the cortex. Stroke, 26:636-643.

Gharbawie OA, Gonzalez CL, Williams PT, Kleim JA, Whishaw IQ (2005) Middle cerebral artery (MCA) stroke produces dysfunction in adjacent motor cortex as detected by intracortical microstimulation in rats. Neuroscience, 130:601-610.

Gharbawie OA, Karl JM, Whishaw IQ (2007) Recovery of skilled reaching following motor cortex stroke: do residual corticofugal fibers mediate compensatory recovery? European Journal of Neuroscience, 26:3309-3327.

Gharbawie OA, Williams PT, Kolb B, Whishaw IQ (2008) Transient middle cerebral artery occlusion disrupts the forelimb movement representations of rat motor cortex. European Journal of Neuroscience, 28:951-963.

Gibb RL, Gonzalez CL, Wegenast W, Kolb BE (2010) Tactile stimulation promotes motor recovery following cortical injury in adult rats. Behavioural Brain Research, [Epub ahead of print].

Gonzalez CL \& Kolb B (2003) A comparison of different models of stroke behaviour and brain morphology. European Journal Neuroscience, 18:1950-1962. 
Gregersen R, Lambertsen K, Finsen B (2000) Microglia and macrophages are the major source of tumor necrosis factor in permanent middle cerebral artery occlusion in mice. Journal of Cerebral Blood Flow and Metabolism, 20:53-65.

Hartings JA, Rolli ML, Lu XCM, Tortella FC (2003) Delayed secondary phase of periinfarct depolarizations after focal cerebral ischemia: relation to infarct growth and neuroprotection. The Journal of Neuroscience, 23:11602-11610.

Hall RD \& Lindhom EP (1974) Organization of motor and somatosensory neocortex in albino rat. Brain Research, 66:23-28.

Hasbani MJ, Schlief ML, Fisher DA, Goldberg MP (2001) Dendritic spines lost during glutamate receptor activation reemerge at original sites of synaptic contact. Journal of Neuroscience, 21:2392-2403.

Hicks SP \& D'Amato CJ (1968) Cell migrations to the isocortex of the rat. Anatomical Record, 160:619-634.

Hsu JE \& Jones TA (2004) Contralesional neural plasticity and functional changes in the less-affected forelimb after large and small cortical infarcts in rats. Experimental Neurology, 201:479-94.

Hughes CC \& Lantos PL (1987) A morphometric study of blood vessel, neuron and glial cell distribution in young and old rat brain. Journal of the Neurological Sciences

Iizuka H, Sakatani K, Young W (1989) Corticofugal axonal degeneration in rats after middle cerebral artery occlusion. Stroke, 20:1396-1402.

Ishii H, Arai T, Morikawa S, Inubushi T, Tooyama I, Kimura H, Mori K (1998) Evaluation of focal cerebral ischemia in rats by magnetic resonance imaging and immunohistochemical analyses. Journal of Cerebral Blood Flow and Metabolism, 18:931-934.

Jacewicz M, Kiessling M, and Pusinelli WA (1986) Selective gene expression in focal cerebral ischemia. Journal of Cerebral Blood Flow and Metabolism, 6:263-272.

Jacobs KM, Donoghue JP (1991) Reshaping the cortical motor map by unmasking latent intracortical connections. Science, 944-947.

Jenkins WM \& Merzenich MM (1987) Reorganization of neocortical representations after brain injury: a neurophysiological model of the bases of recovery from stroke. Progress in Brain Research, 71:249-266.

Jiang Q, Zhang ZG, Ding GL, Silver B, Zhang L, Meng H, Lu M, Pourabdillah-Nejed DS, Wang L, Savant-Bhonsale S, Li L, Bagher-Ebadian H, Hu J, Arbab AS, Vanguri 
P, Ewing JR, Ledbetter KA, Chopp M (2006) MRI detects white matter reorganization after neural progenitor cell treatment of stroke. NeuroImage, 32:1080-1089.

Johansson BB \& Belichenko PV (2002) Neuronal plasticity and dendritic spines: effect of environmental enrichment on intact and postischemic rat brain. Journal of Cerebral Blood Flow and Metabolism, 22:89-96.

Johansson IM, Wester P, Hakova M, Gu W, Secki JR, Olsson T (2000) Early and delayed induction of immediate early gene expression in a novel focal cerebral ischemia model in the rat. European Journal of Neuroscience, 12:3615-3625.

Jones TA (1999) Multiple synapse formation in the motor cortex opposite unilateral sensorimotor cortex lesions in adult rats. Journal of Comparative Neurology, 414:5766.

Jones TA, Chu CJ, Grande LA, Gregory AD (1999) Motor skills training enhances lesion-induced structural plasticity in the motor cortex of adult rats. Journal of Neuroscience, 19:10153-10163.

Jones TA, Kleim JA, Greenough WT (1996) Synaptogenesis and dendritic growth in the cortex opposite unilateral sensorimotor cortex damage in adult rats: a quantitative electron microscopic examination. Brain Research, 733:142-148.

Jones TA \& Schallert T (1994) Use-dependent growth of pyramidal neurons after neocortical damage. Journal of Neuroscience, 14:2140-2152.

Karl JM, Sacrey LR, McDonald RJ, Whishaw IQ (2008) Intact intracortical microstimulation (ICMS) representations of rostral and caudal forelimb areas in rats with quinolinic acid lesions of the medial or lateral caudate-putamen in an animal model of Huntington's disease. Brain Research Bulletin, 77:42-48.

Karl JM, Alaverdashvili M, Cross AR, Whishaw IQ (2010) Thinning, movement, and volume loss of residual cortical tissue occurs after stroke in the adult rat as identified by histological and MRI analysis. Neuroscience, [Epub ahead of print].

Kartje-Tillotson G, O’Donoghue DL, Dauzvardis MF, Castro AJ (1987) Pyramidotomy abolishes the abnormal movements evoked by intracortical microstimulation in adult rats that sustained neonatal cortical lesions. Brain Research, 415:172-177.

Kasamatsu T \& Pettigrew JD (1976) Depletion of brain catecholamines: failure of ocular dominance shift after monocular occlusion in kittens. Science, 194:206-209.

Kasamatsu T \& Pettigrew JD (1979) Preservation of binocularity after monocular deprivation in the striate cortex of kittens treated with 6-hydroxydopamine. The Journal of Comparative Neurology, 185:139-162. 
Kawamura S, Yasui N, Shirasawa M, Fukasawa H (1991) Rat middle cerebral artery occlusion using an intraluminal thread technique. Acta Neurochirurgica, 109:126-132.

Keiner S, Witte OW, Redecker C (2009) Immunocytochemical detection of newly generated neurons in the perilesional area of cortical infarcts after intraventricular application of brain-derived neurotrophic factor. Journal of Neuropathology and Experimental Neurology, 68:83-93.

Kennard MA (1936) Age and other factors in motor recovery from precentral lesions in monkeys. American Journal of Physiology, 115:138-146.

Kinouchi H, Sharp FR, Chan PH, Koistinaho J, Sagar SM, and Yoshimoto T (1994) Induction of c-fos, junB, c-jun, and hsp70 mRNA in cortex, thalamus, basal ganglia, and hippocampus following middle cerebral artery occlusion. Journal of Cerebral Blood Flow and Metabolism, 14:808-817.

Klatzo I (1985) Brain oedema following brain ischaemia and the influence of therapy. British Journal of Anaesthesia, 57:18-22.

Kleim JA, Barbay S, Cooper NR, Hogg TM, Reidel CN, Remple MS, Nudo RJ (2002) Motor learning-dependent synaptogenesis is localized to functionally reorganized motor cortex. Neurobiology of Learning and Memory, 77:63-77.

Kleim JA, Barbay S, Nudo RJ (1998) Functional reorganization of the rat motor cortex following motor skill learning. Journal of Neurophysiology, 80:3321-3325.

Kleim JA, Bruneau R, VandenBerg P, MacDonald E, Mulrooney R, Pocock D (2003) Motor cortex stimulation enhances motor recovery and reduces peri-infarct dysfunction following ischemic insult. Neurological Research, 25:789-793.

Kleim JA, Boychuck JA, Adkins DL (2007) Rat models of upper extremity impairment in stroke. ILAR Journal, 48:374-384.

Kleim JA, Hogg TM, VandenBerg PM, Cooper NR, Bruneau R, Remple M (2004) Cortical synaptogenesis and motor map reorganization occur during late but not early, phase of motor skill learning. Journal of Neuroscience, 21:628-633.

Kobayashi S, Harris VA, Welsh FA (1995) Spreading depression induces tolerance of cortical neurons to ischemia in rat brain. Journal of Cerebral Blood Flow and Metabolism, 15:721-727.

Kokubo Y, Liu J, Rajdev S, Kayama T, Sharp FR, Weinsten PR (2003) Differential cerebral protein synthesis and heat shock protein 70 expression in the core and penumbra of rat brain after transient focal ischemia. Neurosurgery, 53:186-190. 
Kolb B (1987) Recovery from early cortical damage in rats. I. Differential behavioral and anatomical effects of frontal lesions at different ages of neural maturation.

Behavioural Brain Research, 25:205-220.

Kolb B, Brown R, Wit-Lajeunesse A, Gibb R (2001) Neural compensations after lesion of the cerebral cortex. Neural Plasticity, 8:1-16.

Kolb B \& Cioe J (2000) Recovery from early cortical damage in rats, VIII. Earlier may be worse: behavioural dysfunction and abnormal cerebral morphogenesis following perinatal frontal cortical lesions in the rat. Neuropharmacology, 39:756-764.

Kolb B \& Cioe J (2001) Cryoanesthesia on postnatal day 1, but not day 10, affects adult behavior and cortical morphology in rats. Developmental Brain Research, 130:9-14.

Kolb B \& Cioe J (2003) Recovery from early cortical damage in rats. IX. Differential behavioural and anatomical effects of temporal cortex lesions at different ages of neural maturation. Behavioural Brain Research, 144:67-76.

Kolb B, Cioe J, Muirhead D (1998) Cerebral morphology and functional sparing after prenatal frontal cortex lesions in rats. Behavioural Brain Research, 91:143-155.

Kolb B, Cioe J, Whishaw IQ (2000) Is there an optimal age for recovery from motor cortex lesions? I. Behavioral and anatomical sequelae of bilateral motor cortex lesions in rats on postnatal days 1, 10, and in adulthood. Brain Research, 882:62-74.

Kolb B \& Gibb R (1990) Anatomical correlates of behavioural change after neonatal prefrontal lesions in rats. Progress in Brain Research, 85:241-255.

Kolb B \& Gibb R (1991a) Environmental enrichment and cortical injury: behavioral and anatomical consequences of frontal cortex lesions. Cerebral Cortex, 1:189-198.

Kolb B \& Gibb R (1991b) Sparing of function after neonatal frontal lesions correlates with increased cortical dendritic branching: a possible mechanism for the Kennard effect. Behavioural Brain Research, 43:51-56.

Kolb B \& Gibb R (1993) Possible anatomical basis of recovery of function after neonatal frontal lesions in the rat. Behavioral Neuroscience, 107:799-811.

Kolb B, Gibb R, Gorny G, Whishaw IQ (1998) Possible regeneration of rat medial frontal cortex following neonatal lesions. Behavioural Brain Research, 91:127-141.

Kolb B, Gibb R, van der Kooy D (1994) Neonatal frontal cortical lesions in rats alter cortical structure and connectivity. Brain Research, 645:85-97. 
Kolb B \& Holmes C (1983) Neonatal motor cortex lesions in the rat: Absence of sparing of motor behaviors and impaired spatial learning concurrent with abnormal cerebral morphogenesis. Behavioral Neuroscience, 97:697-709.

Kolb B, Holmes C, Whishaw IQ (1987) Recovery from early cortical lesions in rats. III. Neonatal removal of posterior parietal cortex has greater behavioral and anatomical effects than similar removals in adulthood. Behavioural Brain Research, 26:119-137.

Kolb B, Ladowsky R, Gibb R, Gorny G (1995) Does dendritic growth underlie recovery from neonatal occipital lesions in rats? Behavioural Brain Research, 77:125-133.

Kolb B, Morshead C, Gonzalez C, Kim M, Gregg C, Shingo T, Weiss S (2007) Growth factor-stimulated generation of new cortical tissue and functional recovery after stroke damage to the motor cortex of rats. Journal of Cerebral Blood Flow and Metabolism, 27:983-997.

Kolb B, Sutherland RJ, Whishaw IQ (1983a) Abnormalities in cortical and subcortical morphology after neonatal neocortical lesions in rats. Experimental Neurology, 79:223-244.

Kolb B, Sutherland RJ, Whishaw IQ (1983b) Neonatal hemidecortication or frontal cortex ablation produces similar behavioral sparing but opposite effects on morphogenesis of remaining cortex. Behavioral Neuroscience, 97:13-27.

Kolb B \& Tomie J (1988) Recovery from early cortical damage in rats. IV. Effects of hemidecortication at 1, 5, or 10 days of age on cerebral anatomy and behavior. Behavioural Brain Research, 28:259-274.

Kolb B \& Whishaw IQ (1981) Neonatal frontal lesions in the rat: sparing of learned but not species-typical behavior in the presence of reduced brain weight and cortical thickness. Journal of Comparative Physiological Psychology, 32:235-279.

Kolb B \& Whishaw (1985) Earlier is not always better: Behavioural dysfunction and abnormal cerebral morphogenesis following neonatal cortical lesions in the rat. Behavioural Brain Research, 17:25-43.

Kuge A, Takemura S, Kokubo Y, Sato S, Goto K, Kayama T (2009) Temporal profile of neurogenesis in the subventricular zone, dentate gyrus, and cerebral cortex following transient focal cerebral ischemia. Neurological Research, 31:969-976.

Kuhn MJ, Milkulis DJ, Ayoub DM, Kosofsky BE, Davis KR, Taveras JM (1989) Wallerian degeneration after cerebral infarction evaluation with sequential MR imaging. Radiology, 172:179-182.

Kuperberg GR, Broome MR, McGuire PK, David AS, Eddy M, Ozawa F, Goff D, West WC, Williams SC, van der Kouwe AJ, Salat DH, Dale AM, Fischl B (2003) 
Regionally localized thinning of the cerebral cortex in schizophrenia. Archives of General Psychiatry, 60:878-888.

Kury P, Schroeter M, Jander S (2004) Transcriptional response to circumscribed cortical brain ischemia: spatiotemporal patterns in ischemic vs. remote non-ischemic cortex. European Journal of Neuroscience, 19:1708-1720.

Landgren H \& Curtis MA (2010) Locating and labeling neural stem cells in the brain. Journal of Cellular Physiology, 226:1-7.

Landis DM (1994) The early reactions of non-neuronal cells to brain injury. Annual Review of Neuroscience, 17:133-51.

Lashley KS (1931) Mass action in cerebral function, Science, 73:245-254.

Lawyer G, Nesvag R, Varnas K, Frigessi A, Agartz I (2008) Investigating possible subtypes of schizophrenia patients and controls based on brain cortical thickness. Psychiatry Research, 164:254-264.

Le DA, Wu Y, Huang Z, Matsushita K, Plesnila N, Augustinack JC, Hyman BT, Yuan J, Kuida K, Flavell RA, and Moskowitz MA (2002) Caspase activation and neuroprotection in caspase-3-deficient mice after in vivo cerebral ischemia and in vitro oxygen glucose deprivation. Proceedings of the National Academy of Sciences U.S.A, 99:15188-15193.

Lee GA, Lin CH, Jiang HH, Chao HJ, Wu CL, Hsueh CM (2004) Microglia-derived glial cell line-derived neurotrophic factor could protect Sprague-Dawley rat astrocyte from in vitro ischemia-induced damage. Neuroscience Letters, 356:111-114.

Lee JW, Reutens DC, Dubeau F, Evans A, Andermann F (1995) Morphometry in temporal lobe epilepsy. Magnetic Resonance Imaging, 13:1073-1080.

Li Y, Chopp M, Jiang N, Yao F, Zaloga C (1995) Temporal profile of in situ DNA fragmentation after transient middle cerebral artery occlusion in the rat. Journal of Cerebral Blood Flow and Metabolism, 15:389-397.

Lin SP, Schmidt RE, McKinstry RC, Ackerman JJ, Neil JJ (2002a) Investigation of mechanisms underlying transient T2 normalizationin longitudinal studies of ischemic stroke. Journal of Magnetic Resonance Imaging, 15:130-136.

Lin TN, Sun SW, Cheung WM, Li F, Chang C (2002b) Dynamic changes in cerebral blood flow and angiogenesis after transient focal cerebral ischemia in rats: Evaluation with serial magnetic resonance imaging. Stroke, 38:138:145.

Lindquist S \& Craig EA (1988) The heat-shock proteins. Annual Reviews of Genetics, 22:631-677. 
Lindvall O, Ernfors P, Bengzon J, Kokaia Z, Smith ML, Siesjo BK, Persson H (1992) Differential regulation of mRNAs for nerve growth factor, brain-derived neurotrophic factor, and neurotrophin 3 in the adult rat brain following cerebral ischemia and hypoglycemic coma. Proceedings of the National Academy of Sciences U.S.A., 89:648-652.

Lippoldt A, Andbjer B, Rosen L, Richter E, Ganten D, Cao Y, Pettersson RF, Ruxe K (1993) Photochemically induced focal cerebral ischemia in rat: time dependent and global increase in expression of basic fibroblast growth factor mRNA. Brain Researc, 15:45-56.

Liu Y \& Rouiller EM (1999) Mechanisms of recovery of dexterity following unilateral lesion of the sensorimotor cortex in adult monkeys. Experimental Brain Research, 128:149-159.

Longa EZ, Weinstein PR, Carlson S, Cummins R (1989) Reversible middle cerebral artery occlusion without craniectomy in rats. Stroke 20:84-91.

Luke LM, Allred RP, Jones TA (2004) Unilateral ischemic sensorimotor cortical damage induces contralesional synaptogenesis and enhances skilled reaching with the ipsilateral forelimb in adult male rats. Synapse, 54:187-199.

MacDonald E, Van der Lee H, Pocock D, Cole C, Thomas N, VandenBerg PM, Bourtchouladze R, Kleim JA (2007) A novel phosphodiesterase type 4 inhibitor, HT0712, enhances rehabilitation-dependent motor recovery and cortical reorganization after focal cortical ischemia. Neurorehabilitation and Neural Repair, 21:486-496.

Madinier A, Bertrand N, Mossiat C, Prigent-Tessier A, Beley A, Marie C, Garnier P (2009) Microglial involvement in neuroplastic changes following focal brain ischemia in rats. Public Library of Science One, 4:e8101.

McDonald CR, Hagler DJ Jr, Ahamadi ME, Tecoma E, IRagui V, Gharapetian L, Dale AM, Halgren E (2008) Regional neocortical thinning in mesial temporal lobe epilepsy. Epilepsia, 49:794-803.

Memezawa H, Smith ML, Siesjo BK (1992) Penumbral tissues salvaged by reperfusion following middle cerebral artery occlusion in rats. Stroke, 23:552-559.

Mergenthaler P, Dirnagl U, Meisel A (2004) Pathophysiology of stroke: lessons from animal models. Metabolic Brain Disease, 19:151-167.

Merkley TL, Bigler ED, Wilde EA, McCauley SR, Hunter JV, Levin HS (2008) Diffuse changes in cortical thickness in pediatric moderate-to-severe traumatic brain injury. J Neurotrauma, 25:1343-1345. 
Metz GA, Antonow-Schlorke I, Witte OW (2005) Motor improvements after focal cortical ischemia in adult rats are mediated by compensatory mechanisms.

Behavioural Brain Research, 162:71-82.

Mhairi Macrae I (1992) New models of focal cerebral ischemia. British Journal of Clinical Pharmacology, 34:302-308.

Monfils MH, Driscoll I, Vandenberg PM, Thomas NJ, Danka D, Kleim JA, Kolb B (2005) Basic fibroblast growth factor stimulates functional recovery after neonatal lesions of motor cortex in rats. Neuroscience, 134:1-8.

Moon SK, Alaverdashvili M, Cross AR, Whishaw IQ (2009) Both compensation and recovery of skilled reaching following small photothrombotic stroke to motor cortex in the rat. Experimental Neurology, 218:145-153.

Morrison JH, Molliver ME, Grzanna R (1979) Noradrenergic innervation of cerebral cortex: widespread effects of local cortical lesions. Science, 205:313-316.

Nakano S, Kogure K, Fujikura H (1990) Ischemia-induced slowly progressive neuronal damage in the rat brain. Neuroscience, 38:115-124.

Narumiya S, Ohno M, Tanaka N, Yamano T, Shimada M (1998) Enhanced expression of full-length TrkB receptors in young rat brain with hypoxic/ischemic injury. Brain Research, 797:278-286.

Navari RM, Wei EP, Kontos HA, Patterson JL Jr. (1978) Comparison of the open skull and cranial window preparations in the study of the cerebral microcirculation. Microvascular Research, 16:304-315.

Nedergaard M \& Hansen AJ (1993) Characterization of cortical depolarizations evoked in focal cerebral ischemia. Journal of Cerebral Blood Flow and Metabolism, 13:568574.

Nesvag R, Lawyer G, Varnas K, Fjell AM, Walhovd KB, Frigess A, Jonsson EG, Agartz I (2008) Regional thinning of the cerebral cortex in schizophrenia: effects of diagnosis, age and antipsychotic medication. Schizophrenia Research, 98:16-28.

Nowak TS Jr (1985) Synthesis of a stress protein following transient ischemia in the gerbil. Journal of Neurochemistry, 45:1635-1641.

Nowicka D, Rogozinska K, Aleksy M, Witte OW, Skangiel-Kramska J (2008) Spatiotemporal dynamics of astroglial and microglial responses after photothrombotic stroke in the adult rat. Acta Neurobiologiae Experimentalis, 68:155-168.

Nudo RJ (1997) Remodeling of cortical motor representations after stroke: implications for recovery from brain damage. Molecular Psychiatry, 2:188-191. 
Nudo RJ \& Milliken GW (1996) Reorganization of movement representations in primary motor cortex following focal ischemic infarcts in adult squirrel monkeys. Journal of Neurophysiology, 75:2144-2149.

Nudo RJ, Wise BM, SiFuentes F, Milliken GW (1996) Neural substrates for the effects of rehabilitative training on motor recovery after ischemic infarct. Science, 272:17911794.

Nunez JL, Kim BY, Juraska JM (1998) Neonatal cryanesthesia affects the morphology of the visual cortex in the adult rat. Developmental Brain Research, 111:89-98.

Nunez JL, Koss WA, Juraska JM (2000) Hippocampal anatomy and water maze performance are affected by neonatal cryoanesthesia in rats of both sexes. Hormones and Behavior, 37:169-178.

Overgaard K, Meden P (2000) Influence of different fixation procedures on the quantification of infarction and oedema in a rat model of stroke. Neuropathology and Applied Neurobiology, 26:243-250.

Park JS, Bateman MC, Goldberg MP (1996) Rapid alterations in dendrite morphology during sublethal hypoxia or glutamate receptor activation. Neurobiology of Disease, $3: 215-227$.

Paxinos G \& Watson C (1998) The rat brain in stereotaxic coordinates, Academic Press, San Diego.

Preul C, Hund-Georgiadis M, Forstmann BU, Lohmann G (2006) Characterization of cortical thickness and ventricular width in normal aging: a morphometric study at 3 Tesla. J Magn Reson Imaging 24:513-519.

Priller JH, Flugel A, Wehner T, Boentert M, Haas CA, Prinz M, Fernandez-Klett F, Prass K, Bechmann I, de Boer BA, Frotscher M, Kreutzberg GW, Persons DA, and Dirnagle U (2001) Targeting gene-modified hematopoietic cells to the central nervous system: Use of green fluorescent protein uncovers microglial engraftment. Nature Medicine, 7:1356-1361.

Pujol J, Marti-Vilalta JL, Junque C, Vendrell P, Fernandez J, Capdevila A (1990) Wallerian degeneration of the pyramidal tract in capsular infarction studied by magnetic resonance imaging. Stroke, 21:404-409.

Pulsinelli WA, Brierley JB, Plum F (1982) Temporal profile of neuronal damage in a model of transient forebrain ischemia. Annals of Neurology, 11:491-498. 
Ramic M, Emerick AJ, Bollnow MR, O’Brien TE, Tsai SY, Kartje GL (2006) Axonal plasticity is associated with motor recovery following amphetamine combined with rehabilitation after brain injury in the adult rat. Brain Research, 1111:176-186.

Reinecke S, Dinse HR, Reinke H, Witte OW (2003) Induction of bilateral plasticity in sensory cortical maps by small unilateral cortical infarcts in rats. European Journal of Neuroscience, 17:623-627.

Reinecke S, Lutzenburg M, Hagemann G, Bruehl C, Neumann-Haefelin T, Witte OW (1999) Electrophysiological transcortical diaschisis after middle cerebral artery occlusion (MCAO) in rats. Neuroscience Letters, 12:85-88.

Remple MS, Bruneau RM, VandenBerg PM, Goertzen C, Kleim JA (2001) Sensitivity of cortical movement representations to motor experience: evidence that skill learning but not strength training induces cortical reorganization. Behavioural Brain Research, 123:133-141.

Ribeiro Mde C, Hirt L, Bogousslavsky J, Regli L, Baudat J (2006) Time course of aquaporin expression after transient focal cerebral ischemia in mice. Journal of Neuroscience Research, 83:1231-1240.

Richards BA, Chertkow H, Singh V, Robillard A, Massoud F, Evans AC, Kabani NJ (2008) Patterns of cortical thinning in Alzheimer's disease and frontotemporal dementia. Neurobiology of Aging, doi:10.1016/j.neurobiolaging.2007.12.019.

Rosas HD, Hevelone ND, Zaleta AK, Greve DN, Salat DH, Fischl B (2005) Regional cortical thinning in preclinical Huntington's disease and its relationship to cognition. Neurology, 65:745-747.

Rosas HD, Liu AK, Hersch S, Glessner M, Ferrante RJ, Salat DH, van der Kouwe A, Jenkins BG, Dale AM, Fischl B (2002) Regional and progressive thinning of the cortical ribbon in Huntington's disease. Neurology, 58:695-701.

Rosas HD, Salat DH, Lee SY, Zaleta AK, Pappu V, Fischl B, Greve D, Hevelone N, Hersch SM (2008) Cerebral cortex and the clinical expressionof Huntington's disease: complexity and heterogeneity. Brain, 131:1057-1068.

Sadowski M, Morys J, Berdel B, Maciejewska B (1995) Influence of fixation and histological procedure on the morphometric parameters of neuronal cells. Folia Morphologica, 54:219-226.

Salat DH, van der Kouwe AJ, Tuch DS, Quinn BT, Fischl B, Dale AM, Corkin S (2006) Neuroimaging H.M.: a 10-year follow-up examination. Hippocampus, 16:936-945. 
Sawlani V, Gupta RK, Singh MK, Kohli A (1997) MRI demonstration of Wallerian degeneration in various intracranial lesions and its clinical implications, Journal of Neurological Sciences, 146:103-108.

Schallert T, Leasure JL, Kolb B (2000) Experience-associated structural events, subependymal cellular proliferative activity, and functional recovery after injury to the central nervous system. Journal of Cerebral Blood Flow and Metabolism, 20:15121528.

Schiene K, Staiger JF, Bruehl C, Witte OW (1999) Enlargement of cortical vibrissa representation in the surround of an ischemic cortical lesion. Journal of the Neurological Sciences, 162:6-13.

Schlumpf M, Shoemaker WJ, Bloom FE (1980) Innervation of embryonic rat cerebral cortex by catecholamine-containing fibers. Journal of Comparative Neurology, 192:361-376.

Schmued LC \& Hopkins KJ (2000) Fluoro-Jade B: a high affinity fluorescent marker for the localization of neuronal degeneration. Brain Research, 874:123-130.

Schroeter M, Schiene K, Kraemer M, Hagemann G, Weigel H, Eysel UT, Witte OW, Stoll G (1995) Astroglial responses in photochemically induced focal ischemia of the rat cortex. Experimental Brain Research, 106:1-6.

Seo SW, Im K, Lee JM, Kim YH, Kim ST, Kim SY, Yang DW, Kim SI, Cho YS, Na DL (2007) Cortical thickness in single-versus multiple-domain amnesic mild cognitive impairment. Neuroimage, 36:289-297.

Shin HY, Kim JH, Phi JH, Park CK, Kim JE, Kim JH, Paek SH, Wang KC, Kim DG (2008) Endogenous neurogenesis and neovascularization in the neocortex of the rat after focal cerebral ischemia. Journal of Neuroscience Research, 86:356-367.

Shin YW, Yoo SY, Lee JK, Ha TH, Lee KJ, Lee JM, Kim IY, Kim SI, Kwon JS (2007) Cortical thinning in obsessive compulsive disorder. Human Brain Mapping, 28:11281135 .

Shanina EV, Schallert T, Witte OW, Redecker C (2006) Behavioral recovery from unilateral photothrombotic infarcts of the forelimb sensorimotor cortex in rats: role of the contralateral cortex. Neuroscience, 139:1495-1506.

Sigler A, Mohajerani MH, Murphy TH (2009) Imaging rapid redistribution of sensoryevoked depolarization throught existing cortical pathways after targeted stroke in mice. Proceedings of the National Academy of Sciences, USA, 106:11759-11764.

Simmons DM, Swanson LW (2009) Comparing histological data from different brains: sources of error and strategies for minimizing them. Brain Research Reviews, 60:349367. 
Sims NR \& Anderson MF (2002) Mitochondrial contributions to tissue damage in stroke. Neurochemistry, 40:511-526.

Skoglund TS, Pascher R, Berthold CH (1996) Aspects of the quantitative analysis of neurons in the cerebral cortex. Journal of Neuroscience Methods, 70:201-210.

Spanswick SC, Bray D, Zelinski EL, Sutherland RJ (2009) A novel method for reliable nuclear antibody detection in tissue with high levels of pathology-induced autofluorescence. Journal of Neuroscience Methods, 185:45-49.

Speliotes EK, Caday CG, Do T, Weise J, Kovall NW, Finklestein SP (1996) Increased expression of basic fibroblast growth factor (bFGF) following focal cerebral infarction in the rat. Brain Research. Molecular Brain Research, 39:31-42.

Stoll G, Jander S, Schroeter M (1998) Inflammation and glial responses in ischemic brain lesions. Progress in Neurobiology, 56:149-171.

Stroemer RP, Kent TA, Hulsebosch CE (1995) Neocortical neural sprouting, synaptogenesis, and behavioral recovery after neocortical infarction in rats. Stroke, 26:2135-2144.

Sutherland RJ, Whishaw IQ, Kolb B, Becker JB (1982) Cortical noradrenaline depletion eliminates sparing of spatial learning after neonatal frontal cortex damage in the rat. Neuroscience Letters, 32:125-130.

Taub E, Uswatte G, Mark VM, Morris DM (2006) The learned nonuse phenomenon: implications for rehabilitation. Europa Medicaphysica, 42:241-56.

Tetzlaff W, Kobayashi NR, Giehl KM, Tsui BJ, Cassar SL, Bedard AM (1994) Response of rubrospinal and corticospinal neurons to injury and neurotrophins. Progress in Brain Research, 103:271-286.

Thilmann R, Xie Y, Klehues P, and Kiessling M (1986) Persistent inhibition of protein synthesis precedes delayed neuronal death in postischemic gerbil hippocampus. Acta Neuropathologica, 71:88-91.

van der Zijden JP, Bouts MJ, Wu O, Roeling TA, Bleys RL, van der Toorn A, Dijkhuizen RM (2008a) Manganese-enhanced MRI of brain plasticity in relation to functional recovery after experimental stroke. Journal of Cerebral Blood Flow and Metabolism, 28:832-40.

van der Zijden JP, van der Toorn A, van der Marel K, Dijkhuizen RM (2008b)

Longitudinal in vivo MRI of alterations in perilesional tissue after transient ischemic stroke in rats. Experimental Neurology, 212:207-212. 
Von Monakow C (1914) Diaschis. In.Brain and Behavior I: Mood States and Mind. Pribram KH, ed. (1969) Baltimore: Penguin, 27-36.

Waller A (1850) Experiments on the section of the glossopharyngeal and hypoglossal nerves of the frog, and observations of alterations produced thereby in the structure of their primitive fibres. Philosophical Transcripts of the Royal Society of London, 140:423-429.

Watson BD, Dietrich WD, Busto R, Wachtel MS, Ginsberg MD (1985) Induction of reproducible brain infarction by photochemically initiated thrombosis. Annals of Neurology, 17:497-504.

Wegener S, Weber R, Ramos-Cabrer P, Uhlenkueken U, Sprenger C, Wiedermann D, Villringer A, Hoehn M (2006) Temporal profile of T2-weighted MRI distinguishes between pannecrosis and selective neuronal death after transient focal cerebral ischemia in the rat. Journal of Cerebral Blood Flow and Metabolism, 26:38-47.

Wei OY, Huang YL, Da CD, Cheng JS (2000) Alteration of basic fibroblast growth factor expression in rat during cerebral ischemia. Acta Pharmacologica Sinica, 21:296300 .

Whishaw IQ (2000) Loss of the innate cortical engram for action patterns used in skilled reaching and the development of behavioral compensation following motor cortex lesions in the rat. Neuropharmacology, 39:788-805.

Whishaw IQ, Alaverdashvili M, Kolb B (2008) The problem of relating plasticity and skilled reaching after motor cortex stroke in the rat. Behavioural Brain Research, 192:124-136.

Whishaw IQ, O'Connor WT, Dunnett SB (1986a) The contributions of motor cortex, nigrostriatal dopamine and caudate-putamen to skilled forelimb use in the rat. Brain, 109:805-843.

Whishaw IQ \& Pellis SM (1990) The structure of skilled forelimb reaching in the rat: a proximally driven movement with a single distal rotatory component. Behavioural Brain Research, 41:49-59.

Whishaw IQ, Pellis Sm, Gorny B, Kolb B, Tetzlaff W (1993) Proximal and distal impairments in rat forelimb use in reaching follow unilateral pyramidal tract lesions. Behavioural Brain Research, 56:59-76.

Whishaw IQ, Pellis SM, Gorny BP, Pellis VC (1991) The impairments in reaching and the movements of compensation in rats with motor cortex lesions: an endpoint, videorecording, and movement notation analysis. Behavioural Brain Research, 42:7791. 
Whishaw IQ, Sutherland RJ, Kolb B, Becker J (1986b) Effects of neonatal forebrain noradrenaline depletion on recovery from brain damage: performance on a spatial navigation task as a function of age of surgery and postsurgical housing. Behavioral and Neural Biology, 46:285-307.

Williams PT, Gharbawie OA, Kolb B, Kleim JA (2006) Experience-dependent amelioration of motor impairments in adulthood following neonatal medial frontal cortex injury in rats is accompanied by motor map expansion. Neuroscience, $141: 1315-1326$.

Winship IR \& Murphy TH (2009) Remapping the somatosensory cortex after stroke: insight from imaging the synapse to network. The Neuroscientist, 15: 507-524.

Witte OW, Bidmon HJ, Schiene K, Redecker D, Hagemann G (2000) Functional differentiation of multiple perilesional zones after focal cerebral ischemia. Journal of Cerebral Blood Flow and Metabolism, 20:1149-1165.

Wolf T, Lindauer U, Reuter U, Back T, Villringer A, Einhaupl K, Dirnagle U (1997) Noninvasisve near infrared spectroscopy monitoring of regional cerebral blood oxygenation changes during peri-infarct depolarizations in focal cerebral ischemia in the rat. Journal of Cerebral Blood Flow and Metabolism, 17:950-954.

Wurm F, Keiner S, Kunze A,Witte OW, Redecker C (2007) Effects of skilled forelimb training on hippocampal neurogenesis and spatial learning after focal cortical infarcts in the adult rat brain. Stroke, 38:2833-2840.

Xu HT, Pan F, Yang G, Gan WB (2007) Choice of cranial window type for in vivo imaging affects dendritic spine turnover in the cortex. Nature Neuroscience, 10:549551.

Xu XH, Zhang SM, Yan WM, Li XR, Zhang HY, Zheng XX (2006) Development of cerebral infarction, apoptotic cell death and expression of X-chromosome-linked inhibitor of apoptosis protein following focal cerebral ischemia in rats. Life Sciences, 78:704-712.

Xue JH, Yanamoto H, Nakajo Y, Tohnai N, Nakano Y, Hori T, Iihara K, Miyamoto S (2009) Induced spreading depression evokes cell division of astrocytes in the subpial zone, generating neural precursor-like cells and new immature neurons in the adult cerebral cortex. Stroke, 40:e606-613.

Yang M, Gao F, Liu H, Yu WH, Sun SQ (2009) Temporal changes in expression of aquaporin-3, $-4,-5$, and -8 in rat brains after permanent focal cerebral ischemia. Brain Research, 1290:121-132.

Yam PS, Dewar D, MucCulloch J (1998) Axonal injury caused by focal cerebral ischemia in the rat. Journal of Neurotrama, 15:441-450. 
Zepeda A, Sengpiel F, Guagnelli MA, Vaca L, Arias C (2004) Functional reorganization of visual cortex maps after ischemic lesions is accompanied by changes in expression of cytoskeletal proteins and NMDA and GABA(A) receptor subunits. Journal of Neuroscience, 24:1812-1821.

Zhang S, Boyd J, Delaney K, Murphy TH (2005) Rapid reversible changes in dendritic spine structure in vivo gated by the degree of ischemia. Journal of Neuroscience, 25:5333-5338.

Zhao C, Wang J, Zhao S, Nie Y (2009) Constraint-induced movement therapy enhanced neurogenesis and behavioral recovery after stroke in adult rats. The Tohoku Journal of Experimental Medicine, 218:301-308.

Zilles K, Zilles B, Schleicher A (1980) A quantitative approach to cytoarchitectonics. VI. The areal pattern of the cortex of the albino rat. Anatomy and Embryology, 159:335360 .

Zipursky RB, Lim KO, Sullivan EV, Brown BW, Pfefferbaum A (1992) Widespread cerebral gray matter volume deficits in schizophrenia, Archives of General Psychiatry, 49:195-205.

Zong WX, Thompson CB (2010) Necrotic death as a cell fate. Genes and Development, 20:1-15. 